\title{
1 High resolution, serial imaging of early mouse and human liver bud morphogenesis in three
}

2 dimensions

4 Ogechi Ogoke ${ }^{1 * *}$ Daniel Guiggey, ${ }^{1 * *}$ Tala Mon, ${ }^{1 * *}$, Claire Shamul ${ }^{1}$, Shatoni Ross ${ }^{1}$, Saroja Rao ${ }^{3}$, Natesh

5 Parashurama ${ }^{1,2,6^{*}}$

$7{ }^{1}$ Department of Chemical and Biological Engineering, University at Buffalo (State University of New

8 York), Furnas Hall, Buffalo, NY 14260

$10{ }^{2}$ Clinical and Translation Research Center (CTRC), University at Buffalo (State University of New 11 York), 875 Ellicott St., Buffalo, NY 14203

$13{ }^{3}$ Department of Biological Sciences, University at Buffalo (State University of New York), College of 14 Arts and Sciences, 109 Cooke Hall, Buffalo, NY 14260-4610

${ }^{6}$ Department of Biomedical Engineering, University at Buffalo (State University of New York), Furnas

17 Hall, Buffalo, NY 14260

* Corresponding Author

** These authors contributed equally to this work

Running Title: Imaging 3D liver bud morphogenesis

Keywords: liver development, liver imaging, liver organogenesis, hepatic cords, liver bud, septum transversum mesenchyme, liver bud modeling, liver tissue engineering, organ development, gut

26 development, human pluripotent stem cells, human embryonic stem cells, induced pluripotent stem cells,

27 hepatic differentiation

\section{Contact information:}

30 Natesh Parashurama MD PhD, 907 Furnas Hall, Buffalo, NY 14260; Tel: 716-645-1201; Fax: 716-645-

313822 ; e-mail: nateshp@buffalo.edu 


\section{ABSTRACT \\ 2 Background}

3 Liver organogenesis has thus far served as a paradigm for solid organ formation. The developing liver

4 bud is a well established model of organogenesis, and murine genetic studies demonstrate key molecules

5 involved in key morphogenetic changes. However, the analysis of the liver bud is typically limited to 2D

6 tissue sections, which precludes extensive visualization, quantitation, and analysis. Further, the lack of

7 human liver bud data has further hindered our understanding of human liver organogenesis. Therefore,

8 new analytical and visualization approaches are needed to elicit further morphogenetic details of liver

9 organogenesis and to elucidate differences between mouse and human liver bud growth.

\section{Results}

12 To address this need, we focused on high resolution imaging, visualization, and analysis of early liver 13 growth by using available online databases for both mouse (EMAP, Toronto Phenogenomics center) and 14 human (3D Atlas of Human Embryology), noninvasive multimodality imaging studies of the murine 15 embryo, and mouse/human liver weight data. First, we performed three-dimensional (3D reconstructions)

16 of stacked, digital tissue sections that had been initially segmented for the liver epithelium and the septum 17 transversum mesenchyme (STM). 3D reconstruction of both mouse and human data sets enabled 18 visualization and analysis of the dynamics of liver bud morphogenesis, including hepatic cord formation 19 and remodeling, mechanisms of growth, and liver-epithelial STM interactions. These studies

20 demonstrated potentially under-appreciated mechanisms of growth, including rapid exponential growth

21 that is matched at the earliest stages by STM growth, and unique differences between mouse and human 22 liver bud growth. To gain further insight into the exponential liver bud growth that was observed, we 23 plotted volumetric data from 3D reconstruction together with fetal liver growth data from multimodality 24 (optical projection tomography, magnetic resonance imaging, micro-CT) and liver weight data to 25 compose complete growth curves during mouse (E8.5-E18) and human (day 25-300) liver development. 26 For further analysis, we performed curve fitting and parameter estimation, using Gompertzian models, 27 which enables the comparison between mouse and human liver bud growth, as well as comparisons to 28 processes like liver regeneration. To demonstrate the importance of mesenchyme in rapid liver bud 29 growth and morphogenesis in the human liver bud, we performed functional analysis in which human 30 pluripotent stem cell (hPSC)-derived hepatic organoids were used to model collective migration that 31 occurs in vivo, demonstrating that migration is strongly dependent upon mesenchyme.

\section{Discussion}


1 Our data demonstrates improved visualization with 3D images, under-appreciated and potentially new

2 mechanisms of growth, complete liver growth curves with quantitative analysis through embryonic and

3 fetal stages, and a new functional human stem cell-derived liver organoid assay demonstrating

4 mesenchyme- driven collective migration. These data enhance our understanding of liver organogenesis.

\section{BACKGROUND}

7 The global epidemic of chronic end-stage liver disease has garnered increased interest in liver tissue 8 engineering and liver regenerative medicine, including tools for hepatocyte culture and expansion, 9 pluripotent and adult stem cell biology, tissue chips to replace liver functions, in vivo disease modeling, 10 and drug development (Ogoke, Oluwole et al. 2017). The expansion of liver research into these numerous 11 directions has been sustained by fundamental and seminal studies of hepatic, biliary, and hepatic vascular 12 development, reviewed elsewhere (Zorn 2008). These studies detail key molecular and morphogenetic 13 steps in liver organogenesis, which are intricately linked to the liver bud undergoing rapid liver three14 dimensional (3D) growth, leading to rapid growth and eventually the largest internal organ in the body. If 15 these 3D morphogenetic steps can be further revealed through imaging and quantitative analysis, this can 16 augment approaches in which human stem cell-derived organoids are employed to model liver organogenesis (Koike, Iwasawa et al. 2019).

Seminal genetic studies have established major stages of early liver bud morphogenesis and the dynamic molecular underpinnings that drive these steps. The liver bud is a transient, multicellular structure that arises during E8.5 in mice and $\sim$ day 25 in humans, on the scale of 100-200 $\mu \mathrm{m}$ in width and length (Si-Tayeb, Lemaigre et al. 2010). At E8.5, the liver bud contains the liver diverticulum, a ventral out-pocketing of the single cell-layered gut tube, surrounded by a layer of endothelial cells, which itself is surrounded ventrally by the septum transversum mesenchyme (STM) (Si-Tayeb, Lemaigre et al. 2010). At E9.0, the pre-hepatic liver epithelium transitions to a pseudostratified epithelium, and thickens in response to cardiac patterning of foregut endoderm via FGF2 (Ledouarin 1963);(Chen, Tillberg et al. 2015) STM patterning of hepatic endoderm via BMP4 (Rossi, Dunn et al. 2001), and endothelial cells patterning of adjacent foregut endoderm towards hepatic endoderm (Matsumoto, Yoshitomi et al. 2001). Genetic studies demonstrate that transcriptional changes correlate with morphogenetic changes. For example, Foxa2, the first known pioneer transcription factor, is upregulated in hepatic endoderm and primes silent liver genes within hepatic endoderm prior to overt morphogenesis (Lee, Friedman et al. 2005). Hex expression occurs during pseudostratification, prior to hepatic cord formation, which is

32 essential for rapid liver growth (Bort, Signore et al. 2006);(Enzan, Himeno et al. 1997). These hepatic 33 cords form at E9.5 (23-25 somite stage) and are defined as finger-like projections that protrude into the 34 STM, express the master transcription factors Hex (Martinez Barbera, Clements et al. 2000), Prox1 
1 (Sosa-Pineda, Wigle et al. 2000), and Tbx3 (Ludtke, Christoffels et al. 2009), and undergo both EMT 2 (Bort, Signore et al. 2006) and collective cell migration (Cascio and Zaret 1991). These studies 3 collectively demonstrate that hepatic cords form rapidly and have a short half-life. Despite their 4 importance, existing histological studies demonstrate hepatic cords with a wide range of geometry, 5 location, and thickness (Matsumoto, Yoshitomi et al. 2001); (Suzuki, Kanai et al. 2006). How these 6 signaling molecules travel in 3D to activate transcription factors and effect 3D morphogenesis is not as 7 well understood. To our knowledge, the hepatic cords have not been imaged/visualized in 3D at high 8 spatial resolution in murine or human liver bud.

9 The various mesenchymal elements within the liver bud bear a large role in early 3D fetal liver 10 growth and morphogenesis, as borne out by recent scRNA-seq studies (Lotto, Drissler et al. 2020). The 11 STM envelopes the early liver diverticulum in 3D and mediates growth by soluble factor-based induction, 12 leading to transduction of intracellular signals and transcriptional activation. For example, genetic studies 13 of BMP4, Smad2/3 and neurturin (all part of the TGF3 superfamily) (Weinstein, Monga et al. 2001); 14 (Tatsumi, Miki et al. 2007), HEP growth factor (HGF) (Schmidt, Bladt et al. 1995), and ephrins (Cayuso, 15 Dzementsei et al. 2016), all demonstrate a role for the inductive properties of STM. Smad 2/3 (TGFß 16 superfamily) haplo-deficient embryos exhibit severely disrupted liver growth by E14.5, due to both loss 17 of B1-integrin (a known TGFß, target) expression, and mislocalized E-cadherin expression (Weinstein, 18 Monga et al. 2001). HGF knockout studies are mediated by a lack of c-met receptor engagement in 19 hepatoblasts (Schmidt, Bladt et al. 1995). Further, GATA4 -/- embryos lack STM, hepatic cord formation, 20 and subsequent liver development (Watt, Zhao et al. 2007). Knockout of Hlx1, which is strongly 21 expressed within the STM, does not affect early liver specification and hepatic cord formation, but greatly 22 affects 3D liver expansion (Lints, Hartley et al. 1996). Finally, ARF6 knockout disrupts cell membrane 23 polarization, and receptor recycling in liver epithelia (Suzuki, Kanai et al. 2006). In addition to the STM, 24 hematopoietic stem cells, which seed the fetal liver at $\sim$ E11.0, and at 5-6 weeks in human liver, play a 25 role in liver growth. The absence of fetal liver hematopoiesis leads to complex liver morphological effects 26 with mixed phenotypes (Yagi, Deguchi et al. 1998), while affecting liver maturation via the cytokine 27 Oncostatin M (OSM) (Yoshimura, Ichihara et al. 1996), (Kamiya, Kinoshita et al. 1999). Finally, 28 endothelial cells within the developing sinusoids also play a key role in inductive liver growth 29 (Collardeau-Frachon and Scoazec 2008). The absence of endothelial cells in flk -/- mice inhibits liver 30 growth, and in vivo studies of explanted mouse liver bud demonstrate that endothelial-lined, branched 31 microvasculature induces 3D liver bud outgrowth even in the absence of blood flow (Matsumoto, 32 Yoshitomi et al. 2001). Moreover, VEGF-A, HGF, IL-6, and Wnt are collectively secreted from 33 sinusoidal endothelial cells and signal to HEPs in vivo (LeCouter, Moritz et al. 2003). Taken together, 34 these studies convey the strong inductive, role mesenchyme in 3D fetal liver growth and organogenesis, 
1 although imaging/visualization mesenchyme during early liver bud growth has not been achieved 2 (Gordillo, Evans et al. 2015);(Tanimizu and Mitaka 2017);(Zorn 2008);(Ober and Lemaigre 2018). How 3 the STM integrates with the liver tissue in 3D and enables localized collective migration is less well 4 known.

Despite the significance of 3D liver morphogenesis, there have been a lack of studies that 7 only enables 2D rather than 3D visualization of structure, is highly dependent upon tissue preparation and 8 sectioning angle, and exhibits inter-subject variability (Matsumoto, Yoshitomi et al. 2001, Suzuki, Kanai 9 et al. 2006). To assess human fetal liver growth, scientists have used liver weights or liver morphometry 10 (Szpinda, Paruszewska-Achtel et al. 2015) which don't enable visualization at the earliest stages, or 11 noninvasive imaging approaches, like ultrasound (Pardi and Cetin 2006, Chen, Tillberg et al. 2015), 12 which lack spatial resolution to image morphogenesis. Noninvasive imaging of liver bud morphogenesis 13 is challenging to perform in utero due to tissues, fluids, subject motion, and breathing artifacts. 14 Nonetheless, noninvasive embryo imaging approaches (Nieman, Wong et al. 2011) like light sheet 15 microscopy, (Udan, Piazza et al. 2014), ultrasound (Phoon 2006), optical coherence tomography (OCT) 16 (Syed, Larin et al. 2011), micro-computed tomography (CT) (Wong, van Eede et al. 2015), magnetic 17 resonance imaging (MRI) (Turnbull and Mori 2007), photoacoustic tomography (Turnbull and Mori 18 2007), optical projection tomography (Lickert, Takeuchi et al. 2004) have been employed. Overall, 19 dynamic imaging has been challenging, and there is still a need for high spatial resolution $(\sim 1-10 \mu \mathrm{m})$, 20 dynamic (high temporal resolution), and small field of view imaging to image the earliest dynamic events 21 in liver bud morphogenesis.

22 The E-mouse atlas project (EMAP) started with a concerted effort for 3D digital visualization of 23 anatomy, histology, and gene expression during embryogenesis (Baldock, Bard et al. 1992), and the 3D 24 Atlas of Human Embryology project followed (de Bakker, de Jong et al. 2016). We hypothesize that these 25 digital resources, as well as others (Toronto Phenogenomics center, and mouse and human liver volume 26 data) could be used to better image and visualize early liver morphogenesis and/or estimate liver volumes. 27 Analysis of the liver bud is limited typically to 2D tissue sections, which limits visualization and 28 quantitation. For this reason, differences between mouse and human liver organogenesis are poorly 29 understood. Therefore, new approaches are needed to elicit further morphogenetic details of liver 30 organogenesis and to compare mouse and human liver bud growth. We focused on high resolution 31 imaging, visualization, and analysis of early liver growth by using available online databases for both 32 mouse (EMAP, Toronto Phenogenomics center) and human (3D Atlas of Human Embryology), 33 noninvasive multimodality imaging studies of the murine embryo, and mouse/human liver weight data. 34 We demonstrate improved high resolution 3D imaging, under-appreciated and potentially new 
1 mechanisms of growth, and complete liver growth curves with quantitative analysis. We believe that this

2 will improve our understanding of liver organogenesis. This 3D visualization enables further investigation

3 into the multicellular and mesenchyme specific signals necessary for normal liver organogenesis and

4 microarchitecture (Watt, Zhao et al. 2007);(Zhao, Watt et al. 2005);(Margagliotti, Clotman et al. 2007).

5

\section{METHODS}

\section{Mouse liver development data visualization, three-dimensional reconstruction, and analysis}

Aligned and registered image slices from each desired time point were downloaded from eMouseAtlas database and e-Mouse Atlas Project (EMAP, emouseatlas.org), (Baldock, Bard et al. 1992) in WLZ format (Richardson, Venkataraman et al. 2014). An outline of the process of data acquisition and analysis is shown (Figure 1). In this database, embryos were prepared by fixation, clearing, and embedded in wax, sectioned, stained and mounted on slides. Specimens ranged from 244 to 869 slices with $4 \mu \mathrm{m}$ per pixel in the $\mathrm{x}$ and $\mathrm{y}$ dimensions, and $7 \mu \mathrm{m}$ in the $\mathrm{z}$ dimension. The WLZ files were developed from H\&E stains of mouse embryos. Each time point was based on one specimen, for a total of $(n=11)$ mice. Slices were converted to NII format which preserves the scaling of micrometers to pixels for import into ImageJ (https://imagej.nih.gov/). We first located the gut tube within each mouse as a hollow void, extending throughout the specimen. The liver bud starts as the liver diverticulum which is the ventral outpocketing of the gut tube found by locating the liver diverticulum (denoted in the software) surrounded by mesenchyme. We confirmed this is the liver bud by distinguishing it from the lung bud (superior) and ventral and or dorsal pancreatic buds (inferior). A distinct border can be seen of thickened tissue that was identified to be the walls of the liver diverticulum and it and everything inside leading up next to the gut tube was segmented as the liver. The STM is considered to be the surrounding tissue around the thickened epithelial evident from the H\&E slides.

We referenced published immunofluorescence of liver (stained for Hnf4a, and Hex) bud tissue to determine relative tissues boundaries of mesenchyme and developing liver bud (Margagliotti, Clotman et al. 2008);(Bort, Signore et al. 2006), existing textbooks and online resources of mouse and human developmental biology to color-code our data sets and reflect accurately in vivo representation, (Figure 12, Supplemental Figures 1-3) Therefore, using Segmentation Editor, pre-tagged developing liver, septum transverse mesenchyme (STM), and the gut tube, were segmented arbitrarily into green, red, and yellow, respectively. E8.5 contained only pre-segmented areas of the liver diverticulum and gut tube, but not the STM. Each 3D object was generated from different EmouseAtlas specimens (EMA). For the E8.5 mouse Theiler Stage (TS) 13, EMA13 was used, at E9.0 (TS14), EMA14 was used, at E9.5 (TS15), EMA28 was used, and at E10.0 (TS16), EMA38 was used. In these specimens, 3D reconstruction of the existing liver bud was performed using the $3 \mathrm{D}$ data set which enables the selection of gut tube endoderm, 
1 hepatic endoderm, and STM. 3D imaging of the 3D reconstructed mouse liver bud within the whole

2 embryo was unfortunately unable to illustrate significant images of liver bud morphogenesis (Figure 1-2,

3 Supplemental Figure 1-3). To focus the field of view to the liver bud only, the embryo was removed

4 from view. When the STM or gut tube was obscuring the liver bud, the opacity of these tissues was

5 reduced to $50 \%$. Light and shading were at default settings and movies were recorded using the built-in

6 tool of ImageJ 3D Viewer. Movies were created by 2 frames per degree of rotation and exported at $7 \mathrm{fps}$

7 at a resolution of $512 \times 512$ pixels.

8 For calculations of mouse liver volume, we also downloaded volumetric data for MRI and optical

9 projection tomography (OPT) from the eMouseAtlas database and e-Mouse Atlas Project (EMAP,

10 emouseatlas.org) (Baldock, Bard et al. 1992) in WLZ format (Richardson, Venkataraman et al. 2014).

11 This OPT volume data included E9.5 ( $\mathrm{n}=2)$, E10 $(\mathrm{n}=1)$, E10.5 $(\mathrm{n}=2), \operatorname{E11.0}(\mathrm{n}=1)$, E11.5 $(\mathrm{n}=1)$

12 and the MRI volume data included E14.0 $(\mathrm{n}=1)$ and E18.0 $(\mathrm{n}=1)$. In addition to the mouse embryo

13 image stacks obtained from the e-Mouse atlas, we also acquired volumetric data from the Toronto Centre

14 for Chemogenomic Mouse Imaging Centre for E11.5, E12, E13, and E14

15 (http://www.mouseimaging.ca/). This data was obtained using optical projection tomography (OPT) and

16 computed tomography (CT). For OPT data, volume data was obtained for E11.5 $(\mathrm{n}=1)$, E12 $(\mathrm{n}=1)$,

17 E13 $(\mathrm{n}=1)$, and E14 $(\mathrm{n}=1)$. For CT data, volume data was obtained for E15.5 $(\mathrm{n}=35)$. The mouse

18 number obtained from each analysis approach (Table I) and the voxel dimensions (Table II) are shown.

19 The mouse strains were C57/BL6. Using the same procedure, we performed 3D reconstruction followed

20 by calculation of the volume as above.

\section{3D reconstruction and visualization of human liver bud}

23 For the human development, gray image slices of the aligned and registered H\&E stains were downloaded

24 from the 3D Atlas of Human Embryology (3dembryoatlas.com) along with the label files (de Bakker, de

25 Jong et al. 2012). 3D Atlas of Human Embryology utilizes about 15,000 sections, which were digitized

26 and aligned with the software package Amira to create image stacks. Gray and label files are imported

27 into ImageJ using the TrakEM2 plugin and the scaling of micrometers to pixels is set using values

28 provided by the authors. The STM was segmented manually, and visualized from histological sections,

29 whereas for the developing liver and the gut tube we used the pre-existing segmentation. All segmented

30 regions are exported as image stacks in TIFF format. 3D reconstruction was performed by importing the

31 stacks into the 3D Viewer plugin with a smoothness factor of 10. Pixel values for each time point

32 (Carnegie stage) are listed (Table III). Movies were created by 2 frames per degree of rotation and

33 exported at $7 \mathrm{fps}$ at a resolution of $512 \times 512$ pixels. 


\section{Calculation of mouse and human volumes from liver weights}

2 We also obtained mouse and human liver weight data and estimated volumes from weights. Mouse liver

3 weight data was acquired for E13, E14, E15, and E18 (Nomura 1976). Additional mouse liver weight data

4 was acquired from (Kamei, Tsutsumi et al. 1999) and (Song, Luo et al. 2008)for E13 and E14.5

5 respectively.

6 Human fetal volumes ( $\mathrm{n}=69)$ were obtained (Szpinda, Paruszewska-Achtel et al. 2015) in addition to liver

7 weight data $(\mathrm{n}=861)$ (Man, Hutchinson et al. 2016). The human liver weight data were obtained from

8 human fetuses from (Man, Hutchinson et al. 2016) for days 84 through 294. To account for the growth-

9 restricted liver weights in (Man, Hutchinson et al. 2016), the bottom $10^{\text {th }}$ percentile of the data was

10 removed. The bottom $10^{\text {th }}$ percentile was determined by finding the $4^{\text {th }}$ order polynomial regression line

11 for the liver weight data to find points furthest from this curve. Since the noise increases proportionally

12 with the volume, the error for each data point was adjusted by the factor $1 / V$. The 96 points out of the 957

13 data points with the highest negative error were removed from further analysis. By assuming the liver has

14 a density of water $\left(\sim 1 \mathrm{gm} / \mathrm{cm}^{3}\right)$, the liver volume was able to be estimated for each liver weight.

\section{Calculation of volumes and surface area of embryonic structures}

17 Using the 3D Manager plugin within ImageJ, volume and surface area were measured with the Measure

$183 \mathrm{D}$ option after loading the image stacks.

\section{Estimation of cellular density in the gut tube, liver, and STM}

21 For each region, the original histology was examined and imported into ImageJ. Random sections of each

22 region were selected, and the number of cells was counted. This count was divided by the area of the

23 sections. It was assumed that the height of each cell was $10 \mu \mathrm{m}$ and further divided by a height estimation

24 of $10 \mu \mathrm{m}$, to give a value of cells per cubic micron, a single cell volume of $1000 \mathrm{um}^{3}$, as done previously

25 (Semeraro, Cardinale et al. 2013).

\section{Statistical methods, linear regression analysis and Gompertz modeling}

28 Linear, exponential, logistic, and Gompertz regression models were tested as models for our liver 29 development growth data. The Gompertz model was found to produce the highest coefficient of 30 determination $\left(\mathrm{r}^{2}\right)$ and lowest root mean square error (RMSE) for our data and thus was chosen as the 31 model to fit the data. Gompertz has been used as a model for tumor growth (Yang, Gao et al. 32 2020);(Mehrara, Forssell-Aronsson et al. 2013). The Gompertz model can be described as $V=$

$33 V_{o} e^{\frac{b}{a} *\left(1-e^{-a t}\right)}$ where $V_{o}$ is the initial volume, $\mathrm{b}$ and a are constants, and $\mathrm{V}$ is volume with respect to time

34 t. In this analysis, volume estimates for mouse and human livers were plotted against dpc and examined 
1 by non-linear regression analysis against Gompertz growth curve. GraphPad Prism software (GraphPad

2 Software Inc., San Diego, CA) was used to estimate the constants to fit the data as well as find $\mathrm{r}^{2}$-values,

3 RMSE, p-values, and confidence intervals. The parameters were estimated by least squares regression.

4 The sum-of-squares of the distances from the curve was also weighted by $1 / \mathrm{V}$, since the magnitude of

5 error was found to increase proportionally with the magnitude of the volume. This analysis was done for

6 both mouse and human liver growth. The mouse analysis was done with all the multimodality volume

7 estimates $(n=59)$ as well as with the estimated volumes from liver weight data Nomura et al. $(n=10)$

8 (Nomura 1976), Kamei et al. (n=20) (Kamei, Tsutsumi et al. 1999), and Song et al. (n=11) (Song, Luo et

9 al. 2008). The human embryo 3D reconstruction volume estimates $(n=9)$ were analyzed together with

10 measured human fetal volumes (n=69) (Szpinda, Paruszewska-Achtel et al. 2015) as well as liver weight

11 data (n=861) (Man, Hutchinson et al. 2016).

\section{Reagents/materials}

14 DMEM (Thermofisher, Cat. \#: 10569010), FBS (ThermoFisher, Cat. \#: A3160701), Pen-Strep (10000

$15 \mathrm{U} / \mathrm{ml}$ ) (Thermofisher, 15140122), 0.05\% Trypsin-EDTA (Cat. \#: 25300062), Matrigel (MG) Growth

16 factor-free (Cat. \#: 40230), 384-well round bottom, ultra-low attachment spheroid microplates (Corning,

17 Cat. \#:3830) was purchased from Corning. Aurum Total RNA Mini Kit (Cat. \#: 7326820), DNase I (Cat.

18 \#: 7326828), iTaq Universal SYBR Green Supermix (Cat. \#: 1725121), and iScript cDNA Synthesis Kit

19 (Cat. \#: 1708891) were purchased from Bio-Rad. Tissue Culture Treated 24-well plate (LPS Cat. \#:

20 702001), 75-cm² Polystyrene tissue Culture-Treated Flasks (LPS Cat. \#:708003), PCR Plate Covers (LPS

21 Cat. \#: HOTS-100) were purchased from Laboratory Products Sales (LPS). All PCR primers were

22 purchased from either Integrated DNA technologies (IDT), Sigma Aldrich, or Thermofisher. Human

23 foreskin fibroblasts were a obtained as a kind gift from Dr. Stelios Andreadis' lab at the University at

24 Buffalo, State University of New York.

\section{Feeder-free culture (maintenance) of human pluripotent stem cells}

27 We performed experiments using a commercially available induced pluripotent stem cell (iPSC) line 28 (BXS0114 ACS1028 female (ATCC). Human pluripotent stem cells (hPSC) were cultivated at 90\% $\mathrm{N}_{2}$, $295 \% \mathrm{O}_{2}, 5 \% \mathrm{CO}_{2}$, (Tri-gas HERAcell VIOS 160i CO2 incubators) using room temperature mTESR1 30 medium on 6-well tissue culture-treated plates, coated with 1:15 diluted (in DMEM) growth factor free31 matrigel (MG). Wells were coated with MG by adding $1 \mathrm{~mL}$ of diluted MG per well of a 6-well plate, and 32 incubating for $1.5-2 \mathrm{hrs}$. at $37^{\circ} \mathrm{C}$. Excess dilute MG was then removed and the wells were washed with 33 PBS. Cell culture medium was changed every other day. For passaging, the mTESR1 serum-free 34 maintenance medium was removed, $1 \mathrm{~mL}$ of gentle cell dissociation reagent (GDR) (Stem Cell 
1 Technologies) was added for 10-15 minutes, and single cells or clumps of cells were harvested from the

2 dish. Cells were centrifuged 3 minutes at 800-1000 RPM (Eppendorf 5810 table top centrifuge) and

3 resuspended. Cells were frozen in mTESR 1 medium $+5 \%$ DMSO at $-80^{\circ} \mathrm{C}$ overnight, followed by liquid

4 nitrogen cryostorage. Passage number varied between 15-35 for all experiments.

\section{Preparation of stem cell differentiation medium (SFD medium)}

7 Prepared as stated in another publication (Warren et al. 2020). Basal differentiation media for hPSC

8 differentiation to endoderm, gut tube, and liver is comprised of SFD, a serum-free, defined medium based

9 upon additional mouse and human stem cell studies (Gadue, Huber et al. 2006). SFD medium is 10 composed of RPMI basal media containing (75\% IMDM, 25\% Ham's F12, 0.5\% N2 Supplement, $0.5 \%$

11 B27 supplement, $2.5 \mathrm{ng} / \mathrm{ml} \mathrm{FGF2,} \mathrm{1 \%} \mathrm{Penicillin} \mathrm{+} \mathrm{Streptomycin,} \mathrm{0.05 \%} \mathrm{Bovine} \mathrm{Serum} \mathrm{Albumin,} 2$ mM

12 Glutamine, 0.5mM Ascorbic Acid, and 0.4mM Monothioglycerol (MTG)).

\section{Generation of hPSC-derived definitive endoderm}

15 An endoderm protocol was developed specifically for improved survival at low oxygen and improved 16 morphology. In RPMI medium with 1x B27 (no insulin) and 0.2\% KO serum, definitive endoderm from 17 iPSC cells was induced with Activin A (100 ng/ml) and CHIR $(3 \mu \mathrm{M})$ for 1 day, followed by Activin A 18 (100 ng/ml) for 3 more days. Medium was changed daily and 500-750 $\mu \mathrm{L}$ medium was used per well. The $190.2 \% \mathrm{KO}$ serum was added for improved viability at $5 \% \mathrm{O}_{2}$, and higher seeding densities improved 20 culture morphology.

\section{Generation of hPSC-derived hepatic progenitor cells}

23 To promote hepatic differentiation with growth factors, we adopted a protocol from the literature (Takebe, 24 Sekine et al. 2013). Briefly, on day 4 of definitive endoderm induction, Bone morphogenetic protein 4 25 (BMP4, $10 \mathrm{ng} / \mathrm{ml}$ ), and fibroblast growth factor 2 (FGF2, $20 \mathrm{ng} / \mathrm{ml}$ ) were added from days 4-9, and 26 Hepatocyte growth factor (HGF, $10 \mathrm{ng} / \mathrm{ml})$, Oncostatin M (20 ng/ml), and Dexamethasone (100 uM) were 27 added from days 9-12. Cells were assayed by qRT-PCR on day 12.

\section{4-well plate organoid formation}

30 Hepatic progenitor cells, were obtained from hPSCs differentiated on feeder free Matrigel coated plates 31 under low oxygen. To make endoderm spheroids, differentiation media was aspirated from each well of 32 the plate and Accutase was added to each well and allowed to incubate on the cells for 10 minutes at room 33 temperature. Following this, cells suspended in Accutase media were collected into a $15 \mathrm{~mL}$ tube and 34 spun down at $1000 \mathrm{rpm}$ for $5 \mathrm{~min}$ to pellet the cells. The Accutase was then removed and the cells were 
1 re-suspended in serum free differentiation media (SFD) containing Rho-kinas inhibitor (1:1000) and

2 knockout serum (KOSR, 20\%) and counted using a hemocytometer. The total number of cells needed per

3 well was 20,000 so adjustments were done to have an appropriate number of cells for seeding. Cells

4 compacted in 24 hours in 384 ultra low attachment, (ULA) round bottom plates. In the case of co-

5 culturing with stromal cells i.e., human foreskin fibroblasts (HFF), these fibroblasts were first harvested

6 from T-75 cells and counted and adjusted in concentration to $1 \times 10^{6} / \mathrm{mL}$ in supplemented DMEM. These

7 cells were then centrifuged and resuspended in ice-cold Matrigel at the same concentration $\left(1 \times 10^{6} / \mathrm{mL}\right.$ of

8 Matrigel) before being seeded into the same 384 well plates containing the hepatic organoids in

9 suspension $\left(20 \mathrm{uL}, 2 \times 10^{5}\right.$ cells per well).

\section{Analysis of organoid migration and shape}

12 ImageJ was used to analyze the relative properties of the organoids in culture. Collected images were 13 uploaded to the Image J tool. A global scale bar was used per image uploaded before analysis. The length 14 application in Image $\mathbf{J}$ was used to calculate protrusion length of the separate experimental conditions. In 15 addition, the circularity plugin was used to determine the shape alterations of the central organoid over 16 time in culture. Briefly, each organoid image was converted to 8 bit gray scale and then its outline traced 17 to determine the corresponding circularity.

\section{Phase and brightfield microscopy}

20 For phase microscopy, iPSC derived 2D cells and 3D organoid cultures were imaged with a benchtop 21 microscope specifically a Zeiss Axio fluorescence microscope (SE64, 1344x1024 pixel density) equipped

22 with Axiovision Software (v4) and analyzed using Image J.

\section{RNA isolation, reverse transcription (RT) and quantitative polymerase chain reaction}

24 Total RNA was purified with Aurum Total Mini Kit (Bio-Rad) using the spin column method with DNase 251 (Bio-Rad, Hercules, CA) (reconstituted in $10 \mathrm{mM}$ tris) treatment. RNA concentrations were determined 26 by Nanodrop. RNA was converted to cDNA with an RT reaction using the iScript cDNA Synthesis Kit 27 (Bio-Rad), and the mass of RNA was calculated such that 5 ng RNA per well to be run in the qPCR 28 reaction. The RT reaction was performed using 5 minutes at $25^{\circ} \mathrm{C}, 20$ minutes at $46^{\circ} \mathrm{C}$, and 1 minute at $2995^{\circ} \mathrm{C}$. Reactions were then held either at $4^{\circ} \mathrm{C}$ or on ice. We performed $10 \mu \mathrm{L}$ qPCR $(3 \mu \mathrm{L}$ primers at a 30 concentration of $0.3 \mu \mathrm{M}, 1 \mu \mathrm{L}$ nuclease free water, $1 \mu \mathrm{L}$ cDNA, and $5 \mu \mathrm{L}$ supermix) reactions with iTaq 31 Universal SYBR Green Supermix (BioRad) in a 96-well PCR plate (LPS). The qPCR reaction was done 32 in a CFX96 Touch Real-Time PCR Detection System (BioRad). The qPCR reaction consisted of 33 polymerase activation and DNA denaturation at $98^{\circ} \mathrm{C}$ for 30 seconds followed by, 40 to 45 cycles of $98^{\circ} \mathrm{C}$ 
for 15 seconds for denaturation and $60^{\circ} \mathrm{C}$ for 60 seconds for annealing and extension. Melt curve analysis was performed at $65-95^{\circ} \mathrm{C}$ in $0.5^{\circ} \mathrm{C}$ increments at 5 seconds/step. Relative, normalized, gene expression was analyzed using the delta-delta-Ct method (Livak and Schmittgen 2001), with three duplicates per gene tested

\section{Statistics}

8 Data collected was reviewed and analyzed by Microsoft excel. The Student's t test was used to determine 9 statistical differences between two or more independent groups ( $\mathrm{P}$ value set at <0.05).

\section{RESULTS}

\section{D serial imaging of early liver bud morphogenesis}

To image the murine liver bud at high resolution in $3 \mathrm{D}$, we performed $3 \mathrm{D}$ reconstructions of stacked, digital, cleared, hematoxylin and eosin (H\& E) tissue sections from mouse development at key time points (E8.5, E9, E9.5, and E10) and analyzed the 3D images. At each time point, we segmented each tissue section, targeting the liver epithelium, gut tube, and STM. Upon analysis, the liver bud epithelium is distinct and clearly visualized (green), and demonstrates the gut tube (yellow), the STM (red), and the liver bud (green) (Figure 1A, E9.5). Using these representative, segmented sections, together with segmented images for E8.5, E9.0, E9.5, and E10 (Supplementary Figs 1-3), we first reconstructed the entire embryo in $3 \mathrm{D}$, which enabled visualization of the colored liver bud and gut tube (Figure 2A). These images clearly demonstrate growth in the embryo and liver bud, but liver epithelial morphogenesis (green) could not be visualized, whereas the STM (red) and gut tube (yellow) are clearly visualized (Figure 2A). To visualize the liver bud dynamics over time, we reduced the field of view to reveal only the 3D liver bud epithelium, STM, and gut tube (Compare with Figure 2B with 2A). When viewed as part of the gut tube, the E8.5 liver epithelium appears as a triangular sheet of cells (Figure 2B, E8.5) with tube-like and flat sheet-like regions, which we estimate to be approximately $295 \mu \mathrm{m}$ (base) $\mathrm{x}$ $288 \mu \mathrm{m}$ (height) $\mathrm{x} 75 \mu \mathrm{m}$ (thickness). Although there is known to be transitional epithelium with varying cell size in the liver epithelium (Sherwood, Chen et al. 2009) (Supplemental Figure 4, Supplemental Video 1-2), we estimate approximately 3186 cells in the E8.5 liver bud, assuming a single cell volume of $1000 \mathrm{um}^{3}$, as done previously (Semeraro, Cardinale et al. 2013). Our morphogenesis analysis of 3D liver bud demonstrates an elliptical shape at E9.0 in the cranial/anterior direction, with a rapid transition to an elliptical shape in the lateral direction at E9.5-E10.0 (Figure 2B, Compare E9.0, E9.5, and E10). 
Having imaged liver bud remodeling in $3 \mathrm{D}$, our goal was to visualize and analyze microscopic aspects like the hepatic cords and morphogenetic features. We segmented out the gut tube from the images and focused only on the liver bud. With 3D reconstruction and multiple rotated views of the E9.0 liver bud, we observed numerous miniature finger-like projections with dimensions of 1-10 $\mu \mathrm{m}$ in length/ diameter (Figure 3A-C (arrows), Supplementary Video 2). We also observed sheet-like growth, arranged in a series of ridges spaced between 20-30 $\mu \mathrm{m}$, at E9.0, from the superior to the inferior portion of the liver bud (Figure 3A-C, Supplementary Video 2). Since the original specimens range from 244 to 869 aligned (image registered) slices and the $\mathrm{z}$ dimension (height) interslice distance $7 \mu \mathrm{m}$ in the $\mathrm{z}$ dimension, these sheet-like ridges are not a result of imaging artifact or artifacts associated with stacking.

10 They are not a result of interpolation since interpolation was not performed, and they are not due to loss 11 of liver bud tissue during sectioning, since images are based on whole embryo tissue slices. Importantly, 12 the sheet-like ridges do not appear within the gut tube, but solely within the liver tissue, further 13 precluding the above mentioned possibilities, and indicating that these morphogenetic structures were 14 liver bud-specific. At E9.5, we observed slightly larger finger-like projections $(5-10 \mu \mathrm{m})$ (Figure 3D-G, 15 Supplementary Video 3), indicating 3D growth, and sheet-like 3D growth arranged in larger ridges at 16 E9.5 compared to E9.0 (Figure 3E, Supplementary Video 3). Our data at E10 represents liver bud tissue in which liver epithelial cells (hepatoblasts) and mesenchymal cells intermingle. Because of these, the STM cells are included within the green labeled liver tissue. The E10 liver bud demonstrates numerous interconnections cells that form a sponge-like, trabeculated tissue throughout the liver bud, but not in gut tube (Figure 3H-K, Supplementary Video 3-5). To demonstrate variability and repeatability problems in analysis of the mouse liver bud, we demonstrate $n=4$ tissue sections from existing literature for both E9.0 and E9.5, which demonstrate inter-study and intra-study variability (Figures 3L-M). While these studies demonstrate variability, they also validate 3D reconstruction data. Overall, we demonstrate 3D hepatic cords, 3D sheet-like growth pattern at E9.0-9.5, 3D visualization of trabeculation by E10, and variation present within existing studies.

\section{Quantification of 3D liver bud volumetric growth}

The liver bud leads to rapid expansion to result in the largest organ in the body, but it has not been formally determined how rapid embryonic liver bud growth (E8.5-11.5) physically matches with fetal liver growth (E12-18). To determine this mathematical and physical relationship, we employed data not only from 3D reconstructed tissue sections, but also from micro CT, OP imaging, MRI, and data from published liver weights (converting liver weight to volume), to validate our approach. To add additional data at multiple time points, we identified studies that had measured normal fetal liver weights (Nomura 1976);(Kamei, Tsutsumi et al. 1999);(Song, Luo et al. 2008). To convert weight data to volume data, we 
assumed a density equal to water (1 g/mL) (Overmoyer, McLaren et al. 1987); (Xie, Schwen et al. 2016). We performed a growth analysis of the liver by analyzing liver volume growth over time. Combining embryonic and adult data demonstrated a clear, exponential increase in growth over 5 orders of magnitude (Figure 4A, Table IV). This data agrees with our estimation of growth using E8.5 to E18 measurements from 2D images (data not shown). To better visualize these growth changes, we normalized the data (Figure 4B) and performed a nonlinear regression analysis on both the absolute $\left(\mathrm{R}^{2}=0.85\right.$, and $\mathrm{P}<$ $\left.0.001, \operatorname{RMSE}=11.42 \times 10^{9}\right)$ and normalized data absolute $\left(\mathrm{R}^{2}=0.85\right.$, and $\left.\mathrm{P}<0.001, \mathrm{RMSE}=3.29 \times 10^{4}\right)$ with a Gompertz model (Figures 4C-D), an approach used widely in tumor growth studies (Yang, Gao et al. 2020); (Mehrara, Forssell-Aronsson et al. 2013). We additionally wanted to estimate cell count to place our results in greater context. We estimated cell number using an estimated cell size of $\left(10^{3} \mu \mathrm{m}^{3}\right)$ (Semeraro, Cardinale et al. 2013). The cell number was calculated by dividing the liver volumes in our Gompertz model by this estimated cell volume to estimate the number of cells in the liver over time. Interestingly, the cell kinetics model obtained agrees with previous data (Konstantinidis, Giger et al. 2015);(Ema and Nakauchi 2000) for data between E11 and E17 (Figure 4E). This indicates that cell proliferation may play a greater role in the increase in cell volume than hypertrophy in liver development. Furthermore histology sections within the fetal liver) this time period between dpc 12 and dpc 16 do not demonstrate noticeable increases in cell size or cellular hypertrophy (Figure 4F) (Ayres-Silva Jde, Manso et al. 2011). It is possible that the ability of hepatocytes to regenerate in acute regenerative models exhibits proliferative capacity similar to embryonic liver growth. To this answer question, we compared our Gompertz model to the rapid volumetric growth that during acute liver regeneration (Nevzorova, Tolba et al. 2015). When regeneration data was normalized, and shifted to start at the same time as liver bud growth, growth during liver organogenesis was over 20,000 times the growth during regeneration (Figure 4G), suggesting different mechanisms at play. Consistent with this, the volumetric growth rate during acute regeneration was found to be similar to the Gompertzian volumetric model at day 19 to day 23, when liver bud growth rate is decreased by $90 \%$ of the initially rapid rate at E8.5 (data not shown). Overall, we demonstrate a model of growth that links embryonic and fetal liver growth which clearly correlates well with existing data. This model was used to estimate cell kinetics over time as well as liver bud growth compared to liver regeneration.

\section{D imaging of STM-epithelial interactions and growth during murine liver bud development} There is a renewed interest in mesenchyme interactions during liver organogenesis (Lotto, Drissler et al. 2020). Since mouse genetic studies confirm \{Han, 2020 \#125\}; \{Asahina, 2011 \#126\}STM plays a large role in liver growth, we visualized interactions, with a variety of views, between STM and liver at E8.5 (Figure 5A-C, Supplemental Video 6), E9.0 (Figure 5D-F, Supplemental Video 7), E9.5 (Figures 
5G-I, Supplemental Videos 8), and E 10.0 (Figures 5J-K, Supplemental Video 9). Throughout E8.510, we found that in all cases STM envelops the liver bud, particularly at E9-10. We also observed that STM is not present on the dorsal part of the liver bud (Figures 5I and 5L). Finally, the STM appears to increase in size between E8.5-E10. We quantified the relative and absolute growth of the STM. We observed an $8.8 \times 10^{2}$-fold increase in STM volume, as compared to $8.06 \times 10^{2}$-fold increase in liver bud volume, over the same time period (Figure 5A-B). Overall, the data demonstrated that the STM mirrors the growth pattern of the mouse liver bud between E8.5-E10, and continues to envelop the liver bud with 8 time.

\section{D imaging of migrating cords and sheet-like growth in the developing human liver bud}

11 To our knowledge, there are few if any studies of human liver bud growth. Imaging of the human 3D 12 reconstructed liver demonstrates extensive lateral growth on days 28 and 33 (Supplementary Figure 7A13 C), and by day 33, we found that the liver epithelium (green) was indistinguishable from the STM 14 (Supplementary Figure 7C). The day 25 liver bud demonstrates numerous 3D cellular projections 15 consistent with hepatic cords in multiple directions (Figures 6A-C, Supplemental Videos 10-11). 16 Interestingly, we again notice here not only narrow hepatic cords but also prominent sheet-like structures at multiple levels, particularly visible in anterior-lateral-inferior and anterior-lateral-superior views (Figures 6A-C). These cords and sheet-like structures were not due to artifacts of $3 \mathrm{D}$ reconstruction. For example, they are not present in the gut tube images of the same reconstructed images, and data was once again stacked, but not interpolated between tissue sections. These cords and sheets continue to be present at Day 28 (Figures 6D-F, Supplemental Videos 12-13) in multiple views, with a large sheet present in the ventral direction (Figure 6E) with clear layering or stacking of sheet-like projections (Figure 6F). By Day 33, the liver surface has smoothened and greatly enlarged (Figures 6G-H, Supplementary Figures 7-8, Supplemental Videos 14-15). At this time, the liver demonstrates a $3 \mathrm{D}$ ellipsoid shape with a lateral axis of $\sim 3500 \mu \mathrm{m}$ and $\sim 1000 \mu \mathrm{m}$ in the cranial/anterior direction, indicating extensive lateral growth. We imaged inside the 3D reconstructed liver to analyze interconnections, and observed portions of the liver in enclosed spaces, which appear as green as enclosed spaces (Figure 6I, Supplementary Videos 16). We quantified liver growth between day 25 and day 294 using the volume estimations from these 3D reconstructions, as well as published liver volume data (Szpinda, Paruszewska-Achtel et al. 2015) and

30 liver weight data (Man, Hutchinson et al. 2016). We performed a non-linear regression analysis with this 31 data to fit a Gompertz curve $\left(\mathrm{R}^{2}=0.84, \mathrm{P}<0.001\right.$, RMSE $\left.=3.08 \times 10^{6}\right)$ (Figure 6J) and for normalized 32 to day 25 (Figure $6 \mathbf{K})\left(\mathrm{R}^{2}=0.84, \mathrm{P}<0.001\right.$, RMSE $\left.=1.04 \times 10^{3}\right)$. The data demonstrates a $2.41 \times 10^{3}-$ 33 fold change in volume between day 25 and day 50, which is less than the $1.1 \times 10^{4}$-fold change we 34 measure from E9-E14 during mouse development (Compare Figure 6K with Figure 4B). 


\section{D imaging of STM-epithelial interactions during human liver bud development}

3 We reconstructed the liver bud together with STM to image their interactions. We analyzed several views 4 on Day 25 (Figures 7A-E, Supplemental Videos 17-18) and Day 28 (Figure 7F-H, Supplemental 5 Videos 19-20). The human liver bud at Day 25 demonstrates that STM envelops the liver bud and extends

6 all major directions (Figures 7A-E, Supplemental Videos 17-18). The human liver bud at Day 28 7 demonstrates that STM has grown compared to Day 25, however, visually is has now changed, since it is 8 now absent on several surfaces of the liver. The reason is it absent is because cells of the STM have been 9 absorbed into the liver epithelium and cannot be distinguished (Figures 7F-H). We quantified the STM 10 growth compared to the liver growth on Day 25 and Day 28. The STM size increased by 0.54-fold, while 11 the liver increased by 6.8 -fold (Figure 7I).

Epithelial-mesenchymal interactions in an human stem cell-derived organoid model of human liver 14 bud

15 To functionally determine the role of mesenchyme in embryonic liver bud growth (E8.5-E11), we 16 developed an in vitro, hPSC-derived hepatic organoid model which models collective migration from the 17 E8.5 liver bud into the STM, based on our recent study modeling STM and collective migration from cell 18 lines (Ogoke et al. 2020). This model employed hPSC differentiation towards hepatic progenitors, 19 engineering of hPSC-haptic progenitors-derived organoids, and STM modeling with organoids 20 submerged in MG bearing human fibroblasts (HFF) (Figure 8A-B). Gene expression analysis 21 demonstrated significantly increased transcriptional levels of alpha-fetoprotein and albumin (Figure 8D), 22 suggesting day $12 \mathrm{hPSC}$-hepatic progenitors were specified along the liver lineage. Organoid culture in 23 mesenchyme bearing MG + HFF demonstrated distinct cellular protrusions consistent with 3D collective 24 migration that was not present in the absence of HFF (Figure 8E). Image analysis demonstrated 25 significant changes in circularity (morphogenesis) and protrusions (3D Migration) (Figure 8F). Overall, 26 this functional experimental model supports the liver bud imaging data in terms of the role of the STM in 27 boosting morphogenesis and migration during embryonic liver development.

\section{DISCUSSION}

Scientists are particularly interested in modeling liver organogenesis in vivo for basic science, disease 32 modeling, drug development and discovery, and therapeutic purposes, but many questions remain 33 regarding murine and human liver organogenesis. An important aspect of liver organogenesis is, how 34 does the liver become the largest internal organ in only 10 days of mouse fetal development? Both the 35 underlying morphogenetic mechanisms involved (Koike, Iwasawa et al. 2019) and the role for 
1 intercellular, epithelial-mesenchymal signaling in cell migration (Lotto, Drissler et al. 2020) are not well

2 understood. To answer these questions, we focused on serial, high resolution, 3D imaging and

3 quantitative modeling of liver bud morphogenesis in mouse and humans, as well as functional

4 experiments with hPSC-derived hepatic organoids. We imaged, at high resolution, 3D hepatic cord

5 formation, mesenchymal-liver epithelial interactions, and embryonic and fetal liver growth in both mouse

6 and humans. This enabled imaging of both murine and human hepatic cords in 3D, for the first time, and

7 comparison of structural differences. We applied a mathematical modeling approach to develop a

8 predictive model of growth, and used this to model cell kinetics and compare liver bud growth versus

9 liver regeneration. Further, our model demonstrated previously underappreciated exponential liver growth

10 in early embryonic liver growth and our growth curve matched embryonic to fetal liver growth and was

11 validated by existing liver imaging and fetal liver weight data. We identified morphogenetic features

12 associated with growth, including 3D hepatic cord formation, sheet-like growth, and lateral growth. We

13 also demonstrate that the STM increases in size during early fetal liver growth, is highly interconnected

14 with liver epithelium, and that it may have local effects on growth and remodeling, and our functional

15 experiments with hPSC-hepatic organoids and STM modeling support these findings. Overall, we provide

16 several novel findings that contribute to an improved understanding of liver organogenesis.

Embryonic and fetal liver growth establishes the early tissue architecture of the hepatic, biliary,

18 and vascular components of the liver. Our data suggests that the mouse liver undergoes an overall $8.74 \mathrm{x}$

$1910^{4}$-fold-increase in volume (Figure 4B) between E8.5 to E18. Further, we found a $7.5 \times 10^{3}$ change in

20 volume from E9-E14, which corresponds to a $2.5 \times 10^{4}$-fold change in volume between day 25 and day

2156 in humans (Figure 6K). This is in contrast to our data at later stages of liver growth which

22 demonstrates an approximate 7.3-fold increase in volume (Figure 6K) (Szpinda, Paruszewska-Achtel et

23 al. 2015) between day 126 and 210 (18 weeks to 30 weeks). We also utilized this model to estimate cell

24 number over time (Figure 4E), and demonstrate the exponential growth of liver bud compared to the

25 more linear growth of liver regeneration ( Figure 4G). These studies highlight the uniqueness of early

26 liver bud growth. Our data compares favorably to studies of fetal liver proliferative capacity that

27 demonstrate embryonic day 14 (E14) fetal rat hepatocytes (rat F-Heps) are still proliferating after 6

28 months (or the equivalent of 12 human years) with full rat liver repopulation (Dabeva, Petkov et al. 2000).

29 Further, studies of rat E18 F-Heps or phenotypically isolated rat E19 fetal hepatic progenitors were shown

30 more recently to repopulate $\sim 1 / 3$ the liver mass in acute and chronic models (Boylan, Francois-Vaughan

31 et al. 2017);(Bin, Ma et al. 2012). Although the mechanisms by which the exponential increase in liver

32 bud growth occurs are not well understood, our study suggests that that fetal hematopoiesis or cellular

33 hypertrophy are not fully responsible. For example, the most rapid growth occurs initially, from E8.5 to

34 E10.0, a time when fetal liver hematopoiesis is not believed to have fully commenced (Lotto, Drissler et 
1 al. 2020), although at later stages from E10.0-E18, we still observed rapid growth which could be 2 attributable to hematopoiesis. Further, our tissue section analysis from the literature demonstrates no clear 3 evidence of cellular hypertrophy (Figure 4F). Overall, we feel our quantitative modeling approach can 4 lead to further development of improved models of cell kinetics, which match existing liver cell kinetic 5 growth models in the literature (Figure 4E).

6 Further studies to enhance our understanding of growth may involve careful measurements for the 7 development of population kinetic models, including modeling of the proliferative capacity of multiple 8 cell types, cell death pathways, and cellular metabolism. Improved models can also integrate potentially 9 more complex mechanisms involving suppression of proliferation, cell death, and organ size control that 10 regulate growth at fetal liver growth, and potentially be used to understand organoid growth.

A key finding is that we report high resolution imaging of the 3D hepatic cords in humans and 12 mice. Interestingly, we observe hepatic cords at an earlier stage in mice (E9.0) than previously reported 13 (E9.5). This is likely because our technique has a higher spatial resolution than tissue sectioning, and 14 samples the entire volume of the liver, rather than a single section. Our 3D data agrees with existing data 15 and confirms that these cords migrate to form the trabeculated liver by E10.0 (Figure 3H). It remains to 16 be determined how the 3D hepatic cords form the trabeculated liver, as this occurs very rapidly between 17 E9.5 and E10. Thus, improved high temporal and spatial resolution imaging is required between these 18 time points to further elucidate these mechanisms. Our data suggests that the human liver bud generally 19 has more hepatic cords than the mouse, and more predominantly in the lateral directions. This could be 20 because the liver grows even larger in humans, and lateral growth is a key mechanism. Further, we 21 observed these hepatic cords merging into sheets in the human liver, prior to trabeculation, in both mouse 22 and humans. We speculate that this additional "sheet morphogenesis" step may be critical for exponential 23 growth which occurs in the mouse and human liver. Previous studies have used the term "sheet-like" 24 growth in early fetal liver cultures (Hussain, Sneddon et al. 2004), and the "hepatocyte sheets" have been 25 used to describe mature liver architecture. While hepatic cords have been described to form hepatic sheets 26 that flank the sinusoids, it is unclear if "sheet-like morphogenesis" has been identified or imaged. From 27 our images, it is also unclear if there are layers of mesenchyme between the observed sheets, which would 28 be an interesting subject of a future study.

29 Our analysis demonstrates the importance of interactions between the emerging liver epithelium 30 and the STM. Interestingly, our murine data indicates the STM grows at a nearly identical rate to the liver 31 bud between E8.5-E10, and the absolute volume of the STM slightly larger than that of the liver bud from 32 E8-E10. These two facts are not obvious from the approach of traditional tissue sections. While STM 33 expresses inductive signals like BMP, the expanding STM volume indicates the potential complex role of 34 the STM and reciprocal signaling between the hepatic endoderm/hepatoblasts. This is consistent with the 
1 role of GATA6 and GATA4 within the STM, in which GATA6 and GATA4 knockout via tetraploid 2 blastocyst complementation demonstrates loss of STM mass and inhibits early liver bud development 3 (Watt, Zhao et al. 2007);(Zhao, Watt et al. 2005). Our analysis indicates that large portions of the STM 4 are in a remote location from the liver bud, raising questions of what their role is, because if they were 5 secreting substances to liver epithelium, this would take place over very long distances. Further, human 6 liver bud formation suggests that after sheet-like growth, the STM has been obliterated and internalized 7 laterally, while not in the anterior directions. This strongly suggests the STM may play a role in sheet 8 formation and incorporation of the STM into the liver (Figure 7H). However, we can only speculate 9 regarding the mechanisms by which the STM may accomplish this. Another potential role of the STM is 10 in contributing to liver bud remodeling, and not just growth. Ultimately, our objective was to provide a 11 critical analysis of liver bud development in murine and human models. It's quite possible that STM 12 interactions are not equally important in human and mouse development. Here one of our novel findings 13 is that the STM grows with the liver, which may be obvious, but was not previously stated in liver bud 14 literature. Therefore, our modeling points to reciprocal interactions between STM-liver bud interactions 15 that when recreating the process.

16 Our visualization and modeling approach can enhance existing approaches to model 3D liver 17 organogenesis in vitro. Based on our data, it appears that current in vitro models may not fully accurate 18 3D liver bud morphogenesis. With regards morphogenesis we identified that rapid alterations in liver 19 shape, orientation and size (mass) take place, and our data is consistent with recent data indicating that 20 large population of migrating cells at E9.5 (Lotto, Drissler et al. 2020). While we recognize that cues 21 regulating these phenomena might be cell intrinsic, the adjacent tissue, the STM also rapidly changes in 22 parallel with the liver. Thus, our data provides strong evidence for a supportive role of mesenchyme in 23 facilitating liver bud migration and morphogenesis, which might be valuable for in vitro modeling. To 24 support this idea, our liver bud in vitro models, based upon recent work from our lab (Ogoke et al. 2020), 25 strongly support the notion that mesenchymal cells support early migration from organoids, which with 26 further research, could be used to potentially expand liver tissue. Here we have recreated these models 27 with hPSC-derived hepatic organoids (Figure 8E-F) which demonstrate clear evidence of 3D migration. 28 Thus our imaging data and our in vitro models suggest that migration is a critical step in the exponential 29 growth we observed during liver growth, which improve current generations of hepatic organoid models, 30 which we have recently reviewed (Ogoke, Maloy et al. 2021). While our data strongly suggests 31 mesenchyme bearing HFF is required for driving migration/tissue morphogenesis, additional functional 32 assays, mesenchyme cell perturbations, and dynamic transcriptomic and proteomic analysis of 3D tissue 33 models will aid in further elucidating mechanisms in recapitulating embryonic liver growth. 
For our cell kinetics analysis, we estimated cell size to calculate an approximate cell number over time. To make this estimation, we estimated the volume of all cells (hepatic and endothelial progenitors, stellate cells, hematopoietic stem cells, immune cells) to be $1000 \mu \mathrm{m}^{3}$, i.e., average volume of human cells. In terms of the cell volume estimation, it is also important to note that there exists potential non-cell-bearing, intra-organ space in the growing liver. In fact, putative liver stem cells have a diameter of $\sim 10 \mu \mathrm{m}$ so they can be estimated to accommodate $\sim 1000 \mu \mathrm{m}^{3}$ of space (Darwiche and Petersen 2010). Further complicating the cell volume calculation, studies demonstrate that the intercellular distance between hepatoblasts is believed to be a 4-5 $\mu \mathrm{m}$. Another important point is that mature adult hepatocytes have larger volumes $\left(\sim 3000 \mu \mathrm{m}^{3}\right)$, although the cell volumes estimated here refer to the embryonic and fetal liver (Chen, Soto-Gutierrez et al. 2018). Since hepatoblasts are smaller than hepatocytes, the average size of the hepatic cells likely increases with time. Further, endothelial cells are significantly smaller than hepatoblasts (Pandey, Nour et al. 2020); (Marguerat and Bahler 2012)and may have higher packing densities and less intercellular space than hepatocytes. Therefore, although the average cell volume can be more accurately calculated at each time point, we feel justified in estimating $1000 \mu \mathrm{m}^{3}$ cell volume, accounting for all the complicating factors mentioned above. To calculate the exact cell volumes experimentally, one would have to collect embryonic and fetal livers daily between E8.5-E18, perform cellular digestion, perform staining and cell sorting, estimate cell size and cell fraction from flow cytometry data (or some other technique), and then calculate cell number over time. To our knowledge, the cell size throughout embryological to fetal liver development has not been well established. We have illustrated that there are minimal cell diameter changes during liver development from the literature (Fig. 4F). While it is experimentally feasible to calculate cell volumes over time, we felt this approach was outside of the scope of our study, and we have not determined the exact numbers here.

It is important to note that the finger-like projections and/or sheet-like structures we observe in both liver bud imaging and mesenchyme imaging studies could be confounded with artifacts of tissue sectioning or of $3 \mathrm{D}$ reconstruction. Here we discuss several problems pertaining to this issue and try to provide evidence that these factors are not responsible for generating the images that we have obtained. First, both mouse and human data sections were whole embryo sections (not at the organ level) and reconstructed with appropriate voxel size without the use of interpolation. In other words, the 3D slices were stacked on top of each other and at the correct sizes, but no data was created or interpolated between slices. Any smoothing functions in the software were not at the level of pixel size $(\mu \mathrm{m})$ and therefore did not remove the sheet-like structures and protrusions that we observe. Error due to rotation of the slices is

33 minimal, because the object was fixed and sectioned, and images were co-registered in the dataset itself.

34 During sectioning of the tissue, there could be some physical displacement of the tissue, that when 
reconstructed would no longer remain, and this may be responsible for some of the layering effects that can be seen ( Figure 3C and Figure 6B,D). We would expect these errors to be on the scale of the sectioning blade itself. However, the sheet-like structures we observe in the human liver bud, are on the order, of 10-20 $\mu \mathrm{m}$, while thin sectioning blades are on the order of $250 \mu \mathrm{m}$. A simple wrinkling effect can be observed in the mouse and human gut tube (Figure 2B and Figure 6) but sheet-like growth and formal protrusions are not observed in the area of the liver bud, indicating that these processes are likely unique to the liver. It is possible that liver tissue cuts with properties that are different from the gut tube, but these effects would appear more randomly, rather than as ordered structures. While the tissues were cleared before sectioning, this does not explain the effects we observed. Overall, we are comfortable that the findings that we observed are likely to be real. A good illustration of the high spatial resolution of the technique is the imaging of the E10 mouse liver (Figure 2B, Figure 3F-3H). It is very clear that these images are not due to artifacts. Another limitation of our imaging approach is in regards to the fact that STM cells that have integrated into the liver bud between E8.5 and E10. The trabecular pattern we identify within the liver bud at the onset of E8.5, represents the beginning of the primitive sinusoids. Although there is not an established method to quantify STM cells within the liver bud, we estimate it to be 5\%, approximately the same percentage as in the adult liver.

One of the major weaknesses of our study was that there was only $n=1$ mouse and $n=1$ human for each time point analyzed for the 3D reconstruction of embryonic stages of liver bud development. This can only be improved by expanding the data set, which was outside of the scope of this study. The mouse databases we used each have between 1-4 mice for each condition tested, but only 1 data set per time point is available for download. Although we analyze morphological images of a single mouse at each time point, there are many sources of variation even if more mouse numbers had been used. There are many potential factors that cause variation in morphology as reported in the literature (Figure 3L-M), and it is unclear what is due to mouse variation versus technical variation (after tissue collection/dissection). A potential source of mouse variation may be the variation associated with somitestaging of embryological age (for example, E9.5 mice can have a variation in somite number). Further sources of variation may be due to differences in mouse littermates, differences between strains of mice, and phenotypic variation between individual mice. Another source of variability is technical variability. This technical variability could result from differences in mouse embryo isolation, dissection, angles of liver bud sectioning, tissue storage, tissue processing, sectioning, and staining. Because of this extensive

31 variability between E8.5-E10, we feel that our data is in line with the rest of the literature. We feel that 32 although performing more dissections is appropriate, a high amount of variation would be present and 33 needs to be further addressed. 
Our data from digital datasets (EMAP and 3D Embryo Atlas websites) was validated in our study using published imaging and liver weight data. In fact, we obtained tens to hundreds of other data points at later times of mouse development to validate. We employed mouse imaging data from CT, MRI, optical projection tomography, and ultrasound. It is important to note that MRI data at E9.5 was likely overestimated because we were unable to distinguish between liver bud epithelium and STM. In fact, 6 of the 9 MRI-measured liver volumes were higher than the 3D reconstructed volumes from tissue sections, but the trends that we measured were similar. The only other technique to get more reliable and quantitative measurement is by noninvasive imaging of the intact embryo ex vivo or in vivo. However, any removal of the liver bud would essentially destroy it. The problems with ex vivo imaging, assuming the embryo is no longer alive, is that embryo tissues will have lost tension, as well as blood flow, and organs will lose shape. Further, depending on the imaging technique, there can be many confounding 12 factors to getting the high resolution images we have obtained and analyzed, and very likely the liver bud 13 would lose any sources of potential contrast. The problem with in vivo imaging as the only option is that 14 there will be motion artifact, due to both the mother and the embryo, which will cause a loss of spatial 15 resolution, and more confounding layers like extraembryonic tissues (yolk sac) and structure(umbilical 16 vein), that will cause most imaging techniques to lose considerable spatial resolution. Therefore, there cannot be a more quantitative and reliable technique, at a higher resolution, provided our tracing of the organ was accurate, using any existing imaging technology or technique. It is important to note that our approach was validated by our Gompertzian curve fits, which predict rapidly growing tissue initially, which also agrees with our manual measurements of data present in mouse atlas. A final limitation of the study is the relative assignment of pixels towards one versus another tissue group. We carefully reviewed the literature to make evidence-based predictions about the spatiotemporal location of the respective tissues, segmented by color. The gut tube was highlighted in yellow, the liver diverticulum as an outpocketing of the gut tube was highlighted in green. The STM of the liver bud was then highlighted in red. We acknowledge that there could be minor errors using this approach and that more exact methods could be used to confirm these results. However, these errors would not explain the regular structures that we observe and would only contribute minor errors to our volume calculations without changing the trends observed.

In summary, we feel we have contributed new information regarding liver growth, liver volumes,

30 liver morphogenesis and liver-STM interactions at the earliest time points of liver development. However,

31 further work and new tools are needed to elucidate molecular mechanisms by which hepatoblasts and 32 STM interact to result in exponential liver growth. In addition, we will look to perform perturbation 33 studies that explore cell-cell interactions as well as cell identity experiments that provide evidence for the 34 types of cells present at each stage of liver development. With new clearing agents, whole embryos could 
1 be labeled with antibodies followed by wholemount imaging of the immunofluorescence. Multiple

2 antibodies could be used to distinguish liver epithelium and the different cell types of the STM. This

3 alternative approach would provide very high resolution and additional cellular information that has not

4 yet been obtained.

6 CONCLUSION

7 In conclusion, we have identified new aspects of liver growth, liver morphogenesis, and interactions

8 between liver epithelium and STM. Newer techniques can be employed, like CT, MRI, photoacoustic

9 imaging, and optical projection imaging, to improve datasets, and coupling 3D anatomical information to

10 molecular information like gene expression, could be performed using techniques liked expansion

11 microscopy (Chen, Tillberg et al. 2015). Another approach to understand these morphogenetic steps is

12 whole embryo culture at the earliest stages. Overall, we feel understanding and imaging 3D these

13 processes may lead to further insights about how the liver manages to greatly expand its mass while

14 establishing an underlying complex architecture.

17 Table I: Total mouse number obtained from imaging modalities and liver weights.

\begin{tabular}{|l|l|l|l|l|l|l|l|}
\hline dpc & $\begin{array}{l}\text { Total } \\
\text { mouse } \\
\text { number } \\
\text { at each } \\
\text { time }\end{array}$ & $\begin{array}{l}\text { Total mouse } \\
\text { number from } \\
\text { imaging data }\end{array}$ & $\begin{array}{l}\text { Mouse } \\
\text { number } \\
\text { from micro- } \\
\text { CT }\end{array}$ & $\begin{array}{l}\text { Mouse } \\
\text { number } \\
\text { from } \\
\text { MRI }\end{array}$ & $\begin{array}{l}\text { Mouse } \\
\text { number } \\
\text { from tissue } \\
\text { section }\end{array}$ & $\begin{array}{l}\text { Mouse } \\
\text { number from } \\
\text { optical } \\
\text { projection } \\
\text { imaging }\end{array}$ & $\begin{array}{l}\text { Mouse } \\
\text { number } \\
\text { from liver } \\
\text { weight data }\end{array}$ \\
\hline E8.5 dpc & 1 & 1 & 0 & 0 & 1 & 0 & 0 \\
\hline E9.0 dpc & 1 & 1 & 0 & 0 & 1 & 0 & 0 \\
\hline E9.5 dpc & 3 & 3 & 0 & 0 & 1 & 2 & 0 \\
\hline E10 dpc & 2 & 2 & 0 & 0 & 1 & 1 & 0 \\
\hline E10.5 dpc & 3 & 3 & 0 & 0 & 2 & 1 & 0 \\
\hline E11 dpc & 2 & 2 & 0 & 0 & 1 & 1 & 0 \\
\hline E11.5 dpc & 3 & 3 & 0 & 0 & 1 & 2 & 0 \\
\hline E12 dpc & 2 & 2 & 0 & 0 & 1 & 1 & 0 \\
\hline E13 dpc & 32 & 2 & 0 & 0 & 1 & 1 & 1 \\
\hline E14 dpc & 13 & 3 & 0 & 0 & 0 & 0 & 30 \\
\hline E14.5 dpc & 11 & & 0 & 1 & 11 \\
\hline
\end{tabular}




\begin{tabular}{|l|l|l|l|l|l|l|l|}
\hline E15 dpc & 10 & 0 & 0 & 0 & 0 & 0 & 10 \\
\hline E15.5 dpc & 35 & 35 & 35 & 0 & 0 & 0 & 0 \\
\hline E16 dpc & 10 & 0 & 0 & 0 & 0 & 0 & 10 \\
\hline E18 dpc & 12 & 2 & 2 & 1 & 1 & 0 & 10 \\
\hline
\end{tabular}

1

4 Table II. Voxel size information for each mouse data set.

\begin{tabular}{|c|c|c|c|}
\hline Name & Voxel X (micron) & Voxel Y (micron) & Voxel Z (micron) \\
\hline E8.5 H\&E Stain (EMA13) & 4 & 4 & 4 \\
\hline E9.0 H\&E Stain (EMA14) & 4 & 4 & 7 \\
\hline E9.5 H\&E Stain (EMA28) & 4 & 4 & 7 \\
\hline E10 H\&E Stain (EMA38) & 4 & 4 & 7 \\
\hline E10.5 H\&E Stain (EMA48) & 4 & 4 & 7 \\
\hline E10.5 H\&E Stain (EMA49) & 4 & 4 & 7 \\
\hline E11 H\&E Stain (EMA54) & 4 & 4 & 7 \\
\hline E11.5 H\&E Stain (EMA65) & 4 & 4 & 7 \\
\hline E12 H\&E Stain (EMA76) & 4 & 4 & 7 \\
\hline E13 H\&E Stain (EMA78) & 16.5 & 16.5 & 16.5 \\
\hline E14 H\&E Stain (EMA79) & 4 & 4 & 7 \\
\hline E18 H\&E Stain (EMA102) & 13.3 & 13.3 & 14 \\
\hline E9.5 OPT (EMA36) & 6.2 & 6.2 & 6.2 \\
\hline E9.5 OPT (EMA37) & 8 & 8 & 8 \\
\hline E10 OPT (EMA47) & 7.8 & 7.8 & 7.8 \\
\hline E10.5 OPT (EMA53) & 8 & 8 & 8 \\
\hline E11 OPT (EMA64) & 9.9 & 9.9 & 9.9 \\
\hline E11.5 OPT (EMA75) & 11 & 11 & 11 \\
\hline E14 MRI & 4.99 & 4.99 & 4.99 \\
\hline E18 MRI & 4.71 & 4.71 & 4.71 \\
\hline E11.5, 12, 13, 14 OPT & 17.66 & 17.66 & 17.66 \\
\hline E15.5 Micron CT & 27 & 27 & 27 \\
\hline
\end{tabular}

6 Table III. Voxel size for human studies. 


\begin{tabular}{|l|l|l|l|l|}
\hline Day & $\begin{array}{l}\text { Voxel X } \\
\text { (micron) }\end{array}$ & $\begin{array}{l}\text { Voxel Y } \\
\text { (micron) }\end{array}$ & $\begin{array}{l}\text { Voxel Z } \\
\text { (micron) }\end{array}$ & $\begin{array}{l}\text { Number of } \\
\text { Slices }\end{array}$ \\
\hline $\mathbf{2 5}$ & 0.862 & 0.862 & 8.694 & 283 \\
\hline $\mathbf{2 8}$ & 2.969 & 2.969 & 10.31 & 277 \\
\hline $\mathbf{3 3}$ & 5.885 & 5.885 & 16.55 & 247 \\
\hline $\mathbf{3 7}$ & 10.1209 & 10.1155 & 10.06 & 651 \\
\hline $\mathbf{4 1}$ & 5.2600 & 5.2600 & 20.00 & 547 \\
\hline $\mathbf{4 4}$ & 4.4790 & 4.4790 & 10.00 & 1059 \\
\hline $\mathbf{5 2}$ & 4.4800 & 4.4800 & 60.00 & 288 \\
\hline $\mathbf{5 6}$ & 24.8004 & 24.8004 & 144.3 & 208 \\
\hline
\end{tabular}

2 Table IV: Liver Volume Estimation.

\begin{tabular}{|r|r|r|}
\hline Embryo development stage (dpc) & Liver Volume $\left.\mathbf{( c m}^{\mathbf{3}}\right)$ & Normalized Liver Volume \\
\hline 8.5 & 346944 & 1 \\
\hline 9 & 1403808 & 4.046209186 \\
\hline 9.5 & 1396752 & 4.02587161 \\
\hline 9.5 & 2939537 & 8.472655529 \\
\hline 9.5 & 4059648 & 11.70116215 \\
\hline 10 & 4357696 & 12.56022874 \\
\hline 10 & 7255136 & 20.91154768 \\
\hline 10.5 & 28412384 & 81.89328537 \\
\hline 10.5 & 56539648 & 162.9647666 \\
\hline 10.5 & 25333060 & 73.01772044 \\
\hline 11 & 45120791 & 130.0520862 \\
\hline 11 & 88646690 & 255.5072 \\
\hline 11.5 & 172017664 & 495.8081535 \\
\hline 11.5 & 178403247 & 514.2133803 \\
\hline 11.5 & 390000000 & 1124.100719 \\
\hline 12 & 267186976 & 770.1155691 \\
\hline 12 & 503000000 & 1449.801697 \\
\hline 13 & 5206585434 & 15006.99085 \\
\hline 13 & 3233000000 & 9318.506733 \\
\hline 14 & 10457620839 & 30142.10028 \\
\hline 14 & 10757000000 & 31005.00369 \\
\hline 14 & 25722000000 & 74138.76591 \\
\hline 15.5 & 21995458967 & 63397.72115 \\
\hline 15.5 & 25833171873 & 74459.19766 \\
\hline
\end{tabular}




\begin{tabular}{|r|r|r|}
\hline 15.5 & 21221110003 & 61165.80775 \\
\hline 15.5 & 21776170647 & 62765.66433 \\
\hline 15.5 & 23398227120 & 67440.93318 \\
\hline 18 & 30256430416 & 87208.39794 \\
\hline 18 & 40944000000 & 118013.2817 \\
\hline
\end{tabular}

\section{ABBREVIATIONS}

9 2D - two-dimensional

$10 \quad 3 D$ - three-dimensional

11 EMAP - emouse atlas project

12 STM - septum transversum mesenchyme

13 HEP - hepatocytes

14 FGF - fibroblast growth factor

15 BMP4 - bone morphogenetic protein 4

16 FOXA2 - forkhead box 2

17 Hex - homeobox protein

18 Prox1 - prospero homeobox 1

19 Tbx3 - T-box transcription box factor 3

20 EMT - epithelial to mesenchymal transition

21 TGFB - transforming growth factor beta

22 ARF6 - ADP-ribosylation factor 6

23 VEGF-A - vascular endothelial growth factor

24 HGF - hepatocyte growth factor

25 IL-6 - interleukin 6

26 HNF4a - hepatocyte nuclear factor 4 alpha

27 GATA6 - GATA Binding Protein 6

28 GATA4 - GATA Binding Protein 4 
bioRxiv preprint doi: https://doi.org/10.1101/803478; this version posted May 15, 2021. The copyright holder for this preprint (which was not certified by peer review) is the author/funder. All rights reserved. No reuse allowed without permission.

1 CT - computed tomography

2 MRI - magnetic resonance imaging

3 
A. Image Analysis/ Segmentation
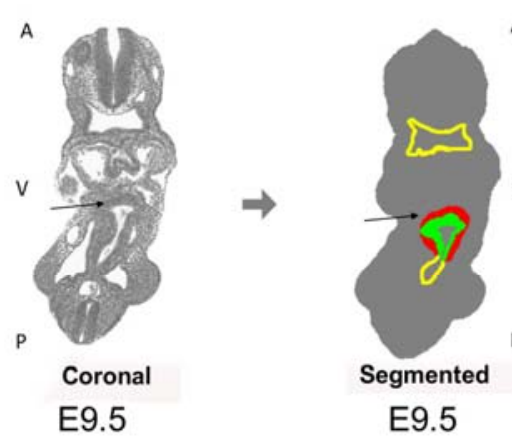

B. Three-dimensional reconstruction
A

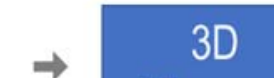

Manager
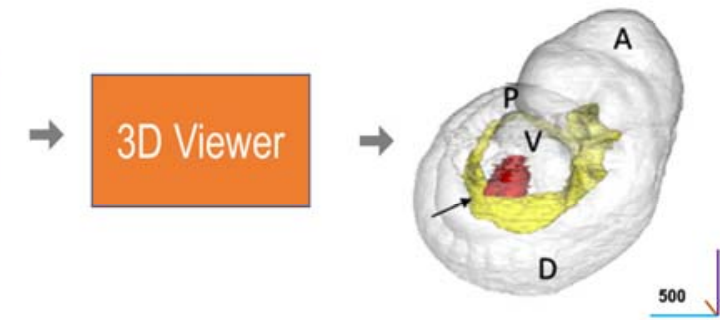

Figure 1. Figure 1. Data processing for 3D reconstruction of mouse liver bud. Listed are the steps in acquiring imaging data, segmenting data, and performing 3D reconstruction. 1) Access emouseatlas.org, mouse anatomy atlas, 2) Choose dataset and time point, 3) Click on download, obtain zip file wlz format of the embryo, 4) Download Woolz from EMAP/Analysis Tools \& Resources/Software in the main menu bar 5) Use WlzExtFFConvert from the command line to convert wlz to nii format 6) Import nii format into ImageJ. A similar process was used for human liver as described in methods.

A) At this stage, a cleared, sectioned $\mathrm{H} \& \mathrm{E}$ stained embryo is visualized.The image is opened in "Segmentation Editor" and the key parts of the liver bud, mesenchyme, and gut tube are segmented using the mouse atlas with unique colors, using previous liver bud papers or mouse altals as a guide. The label file is saved.

B) The 3D Manger (plugin) is used to visualize 3D structure (3D Viewer) and 3D measure is used to perform analysis (i.e. volume).

C) 3D reconstruction is performed using 3D viewer with a smoothness factor of 10 . Data was checked against mouse atlas and other studies of liver development to ensure accuracy. Scale bar unit is in microns. 

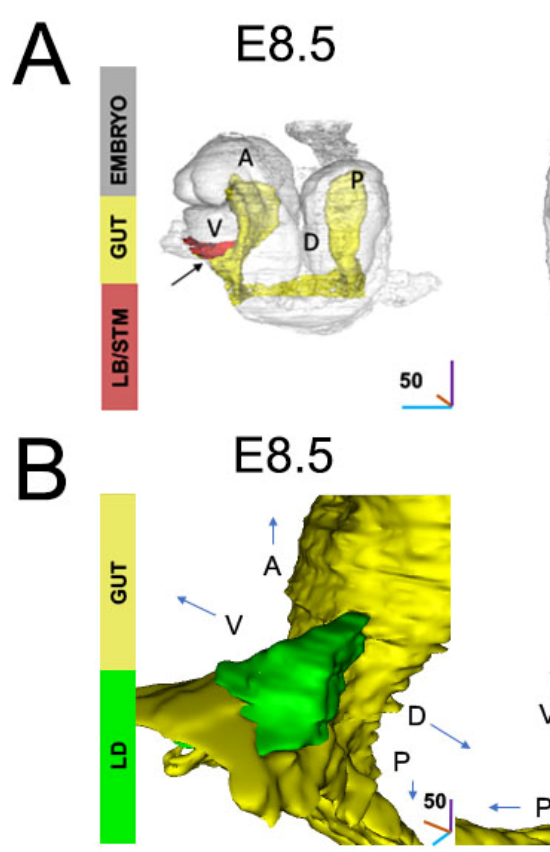
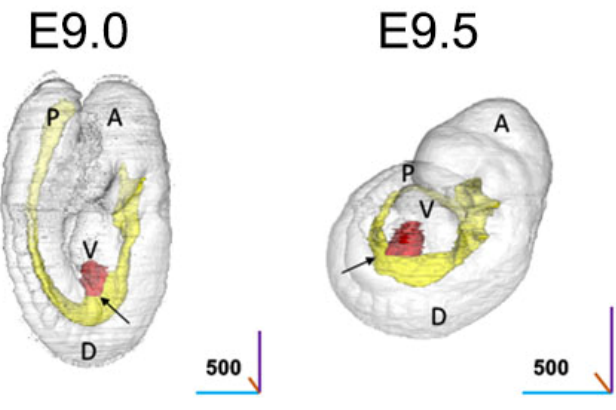

E10

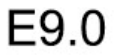

E9.5
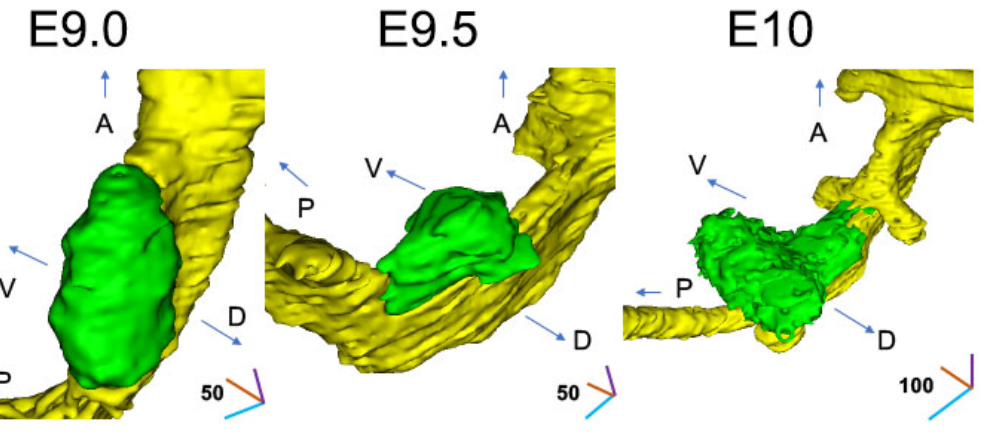

Figure 2. 3D reconstruction and visualization of the developing murine liver bud.

To dynamically visualize morphogenetic stages during mouse liver bud formation, 3D datasets, consisting of cleared hematoxylin and eosin ( $\mathrm{H} \& \mathrm{E}$ ) tissue sections from mouse development were used. Datasets were reformatted, imported, segmented, and reconstructed in 3D. Ventral views are shown. A = anterior, $\mathrm{V}=$ ventral, $\mathrm{P}=$ posterior. Arrows depict directions, gut tube (yellow), the liver bud (green). One specimen was used for each time point.

A) Whole body 3D reconstruction of the developing embryo at transient times of development (E8.5, E9.0, E9.5,E10). The major components of the murine liver bud are highlighted in separate colors. Gut tube (yellow), and Liver bud (LB) and septum transversum mesenchyme (STM) in red. Arrows (black) indicate location of the LB/STM. Scale bar in first panel is in $\mu \mathrm{m}$. Scale bar in next three panels is in microns.

B) 3D reconstruction of the posterior foregut focused on the liver murine liver bud, at E8.5 from Theiler Stage (TS) 13. Liver epithelium initially appears as a triangular sheet of cells with a base of $25 \mu \mathrm{m}$ At E9.0 (TS14)gut tube has closed with the liver bud, forming an elliptical shape tubelike structure with long axis in the cranial-anterior direction. For E9.5 (TS15) the liver bud remodels its shape, with an elliptical long axis in the lateral direction. At E10.0 (TS16). Liver bud remodeling with an elliptical long axis extending bi-laterally. At this stage, the axis has completely changed from E9.0. The liver surface appears more roughened. 

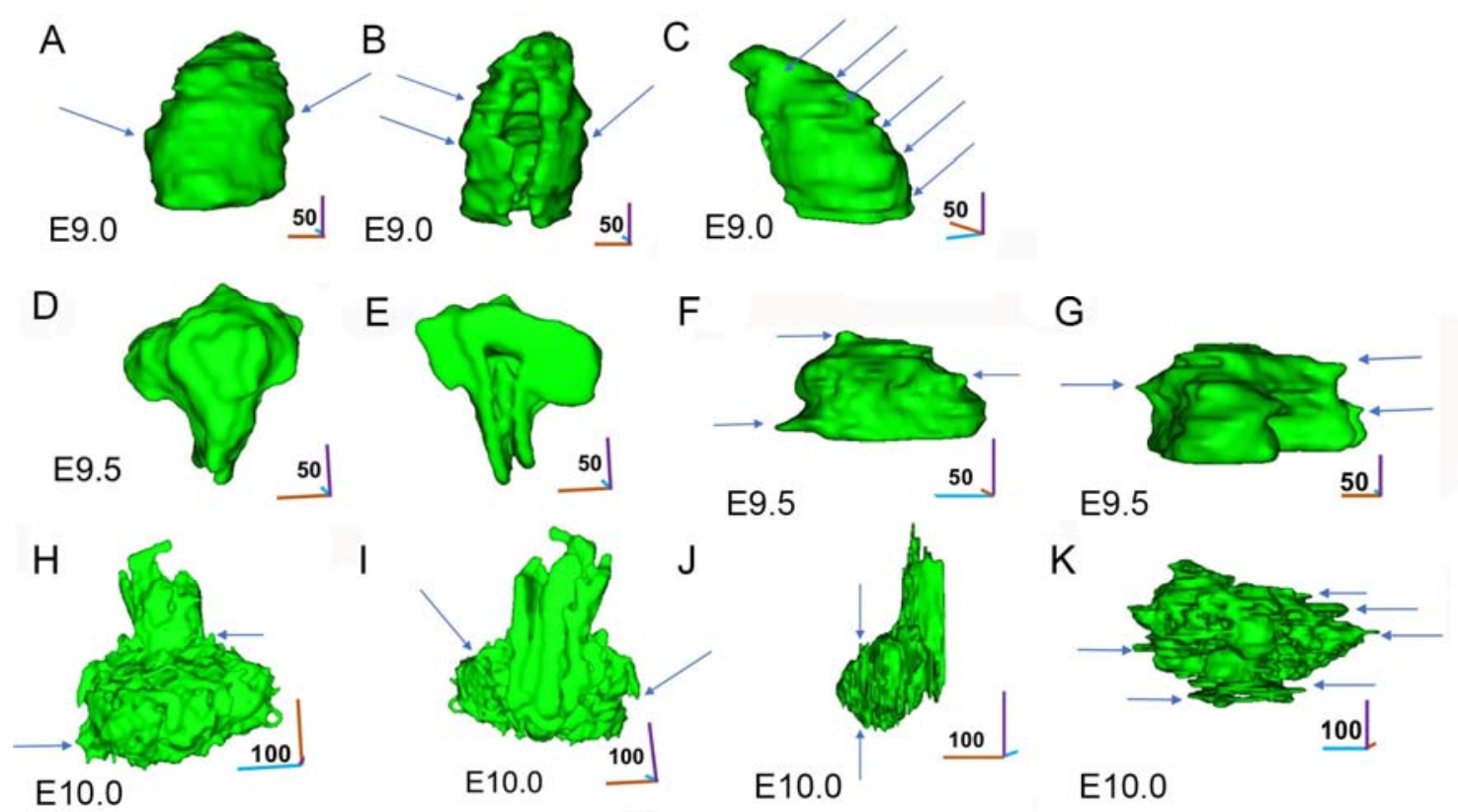

E10.0
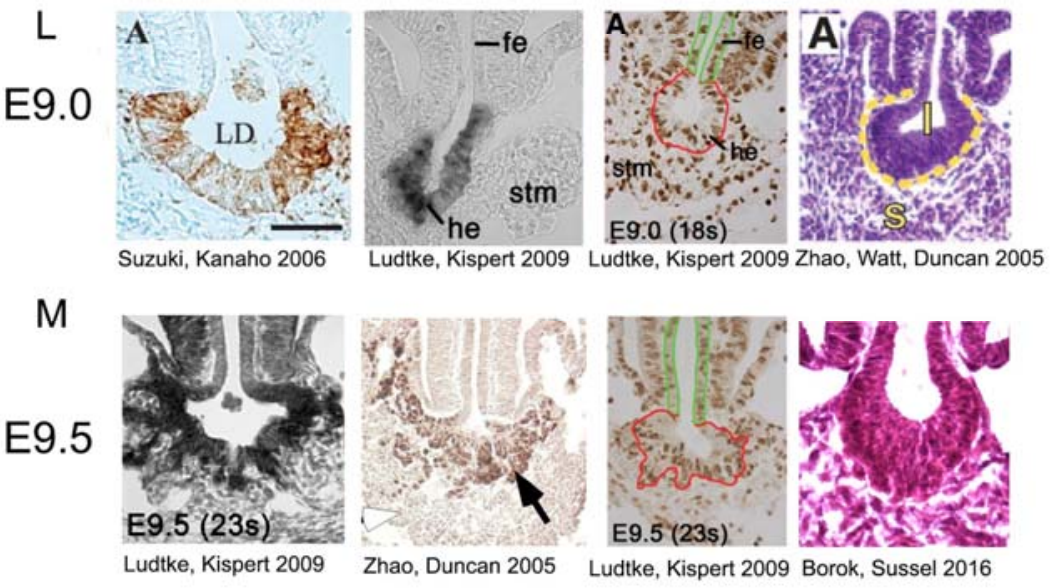

Figure 3. Multiple views of 3D murine liver bud demonstrate dynamic formation of cord-like structures and sheet-like growth.

A) 3D reconstruction of the 9.0 murine liver bud, ventral view. Arrows demonstrate multiple early hepatic cords which demonstrate migration in the lateral directions.

B) Same structure as A), except dorsal view. The dorsal view demonstrates how the liver bud wraps around the gut tissue (which is absent), with an associated lumen. The associated hepatic cords are depicted by arrows.

C) Same structure as A), except right lateral view. In this case, arrows depict not hepatic cords, but sheet-like growth at various levels in the cranial-anterior axis. This sheet-like growth appears most prominently in this view.

D) 3D reconstruction of the E9.5 murine liver bud (TS15), ventral view.

E) Same structure as D), except dorsal view.

F) Same structure as D), except dorsal view, inferior view. Blue arrows depict hepatic cords, while sheet-like growth is not observed.

G) Same structure as D), except left lateral view. Blue arrows depict hepatic cords, while orange arrows depict sheet-like growth. 
H) 3D reconstruction of the E10.0 murine liver bud (TS16). Arrows depict examples of migrating cords. The liver bud now demonstrates a trabeculated tissue with interconnecting open-spaces.

I) Same structure as $\mathrm{H}$ ), except dorsal view

J) Same structure as H), except left lateral view, demonstrating trabeculated tissue. Arrows depict potential hepatic cords.

K) Same structure as H), except inferior view, demonstrating trabeculated tissue. Arrows depict potential hepatic cords.

L) Cross sections of mouse liver bud at E9.0 as reported in the literature. 4 examples given with references below.

M) Cross sections of liver bud at E9.5 as reported in the literature. 4 examples given with references below. 

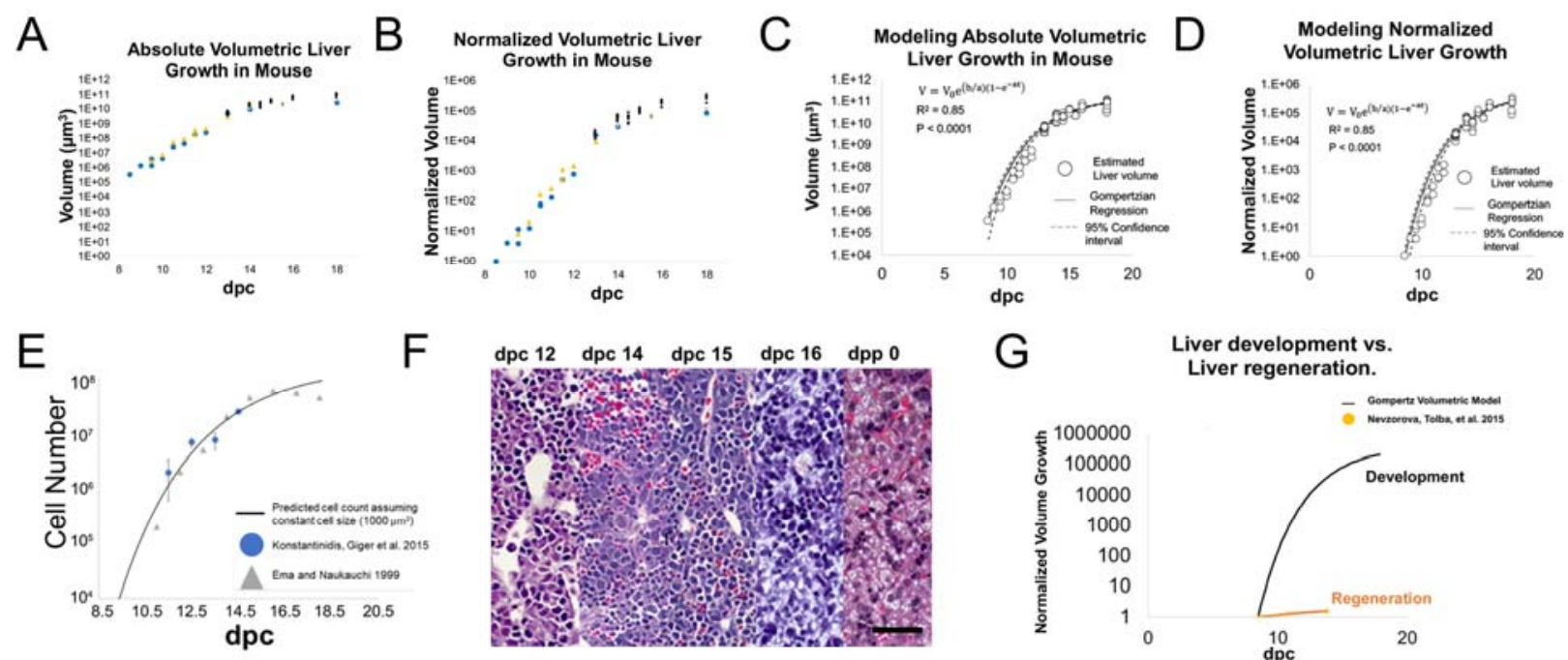

Liver development vs. Liver regeneration.

$\mathrm{H}$

$H$
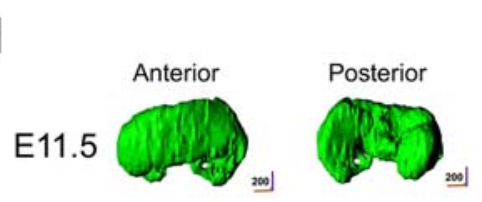

I
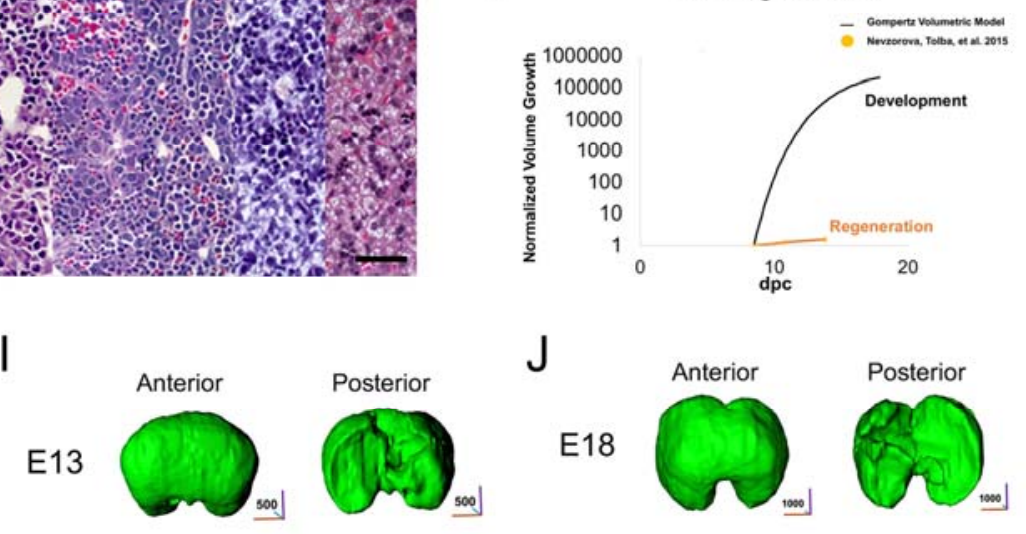

Figure 4. A quantitative model of volumetric growth of the murine developing liver. Since images demonstrated rapid liver bud growth, volumetric growth was quantified from E8.5-11.5 (embryonic stage) and E12-18 (fetal liver stage). Volumetric data was obtained from 3D reconstruction of tissue slices, MRI, optical projection imaging and liver weights. E8.5-E18 $(n=1, E 8.5 ; n=1, E 9 ; n=3, E 9.5 ; n=2, E 10 ; n=3$, E10.5, n=2, E11, n=3, E11.5; n=2, E12; n=32, E13; n=13, E14; n=11, E14.5; n=10, E15; n=35, E15.5; $\mathrm{n}=10, \mathrm{E} 16 ; \mathrm{n}=12, \mathrm{E} 18)$. An observed $10^{5}$-fold increase in liver volume from E8.5 to E18.

A) Absolute volume of the reconstructed 3D liver murine liver. Plotted points are identified in different colors based on source of data: optical projection (yellow), magnetic resonance imaging (gray), tissue section (blue), micro-CT (green) and liver weight (black).

B) Relative liver volume was calculated by normalizing to the liver embryo volume at E8.5. Plotted points are identified in different colors based on source of data: optical projection (yellow), magnetic resonance imaging (gray), tissue section (blue), micro-CT (green) and liver weight (black).

C) Absolute liver volume changes are modeled using a non-linear regression Gompertzian model Absolute liver volume changes are modeled using a non ${ }^{\frac{b}{a}} *\left(1-e^{-a t}\right)$. Assuming $95 \%$ confidence

D) Relative liver volume was calculated by normalizing to the corresponding embryo volume. interval ), $V_{o}=1.0 \mathrm{E}-72 ; \mathrm{a}=0.3069,0.3245 ;$ and $\mathrm{b}=58.16,61.40 ; \mathrm{RMSE}=11.42 \times 10^{9} . \mathrm{R}^{2}=$ 0.85 , and $\mathrm{P}<0.001$. Changes are modelled using a non-linear regression Gompertzian model of tissue growth and fitted to the equation $V=V_{o} e^{\frac{b}{a} *\left(1-e^{-a t}\right)}$. Assuming 95\% confidence interval, $V_{o}=2.9 \mathrm{E}-78$, a $=0.3069,0.3245$; and $\mathrm{b}=58.16,61.40 ; \mathrm{RMSE}=3.29 \times 10^{4} . \mathrm{R}^{2}=0.85$ and $\mathrm{P}<0.001$.

E) Cell count estimated with the previously derived volumetric Gompertz model and assuming a fixed cell volume of $1000 \mu \mathrm{m}^{3}$. Cell count data included from Konstantinidis et al. as blue circles. Each marker represents the mean and standard deviation of at least 5 fetal livers for each time point. Additional data also included from Ema and Naukauchi as gray triangles. Each marker is the mean of 7 or 8 fetal livers except dpc 11, which is the average of 31 fetal livers. 
1 F) H\&E Stains of a mouse fetal liver at dpc 12, 14, 15, 16, and dpc 0(Ayres-Silva Jde, Manso et al. 2011). Scale Bar $=30 \mu \mathrm{m}$.

3 G) A comparison of the relative volumetric growth for the Gompertz model and liver regeneration data from a partial hepatectomy (Nevzorova, Tolba et al.). Data was normalized to E8.5 in the Gompertz model and lowest liver weight after partial hepatectomy.

H) 3D reconstruction of the liver bud at E11.5, E13, and E18 (green). Anterior and posterior views of the developing liver. 


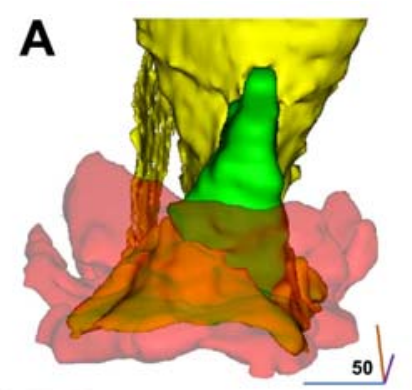

B

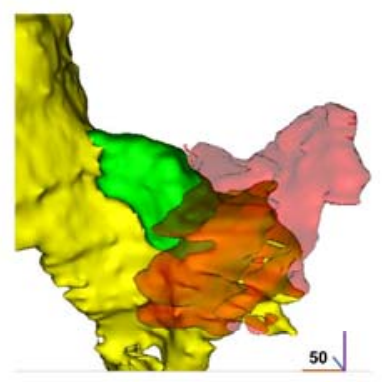

C

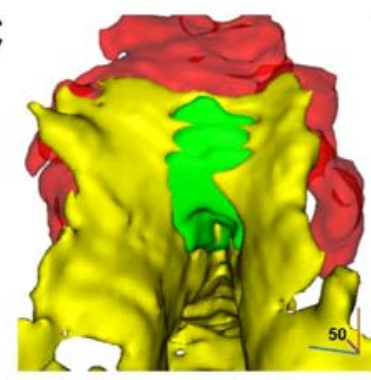

\section{E 8.5}
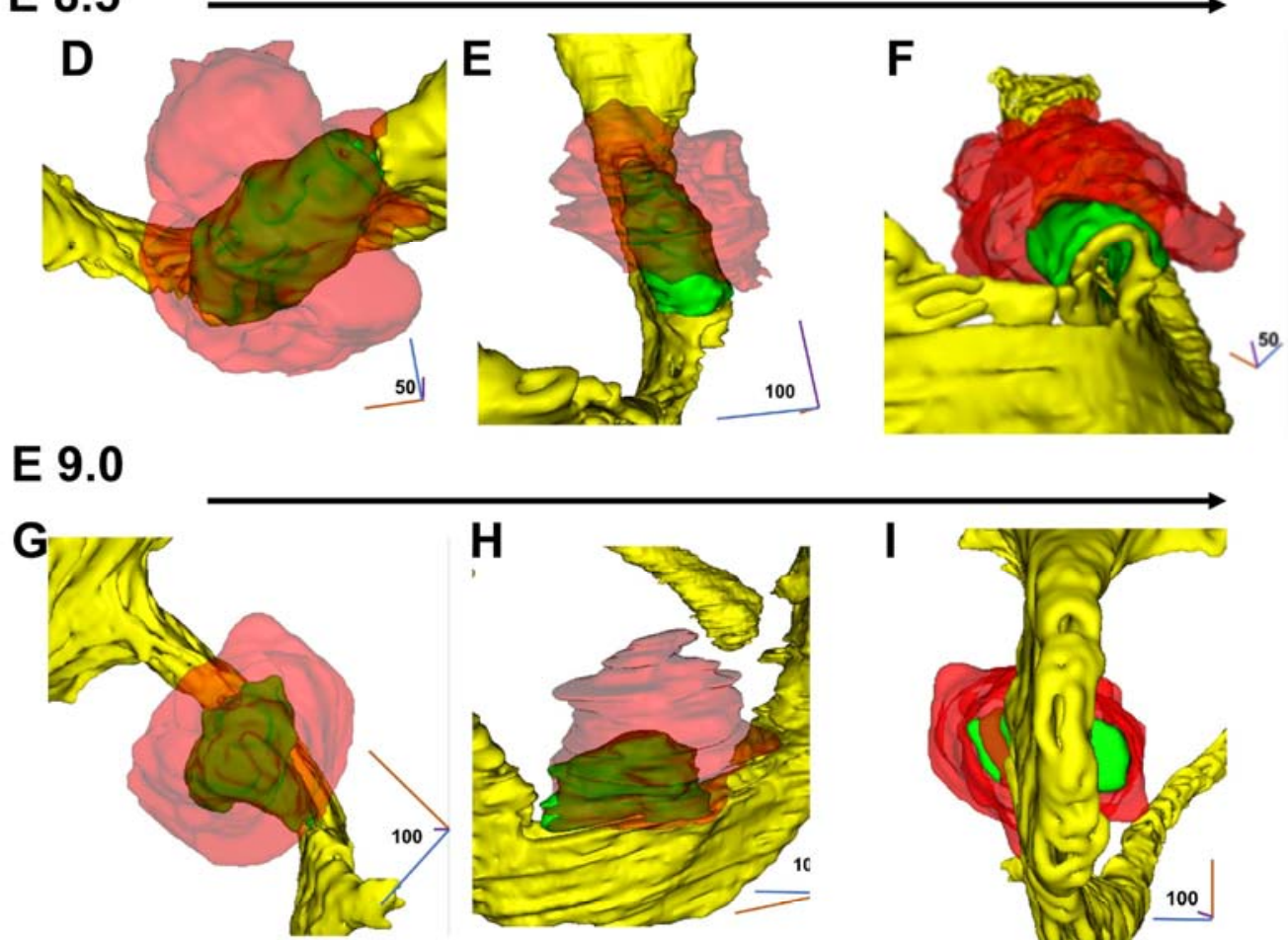

\section{I}
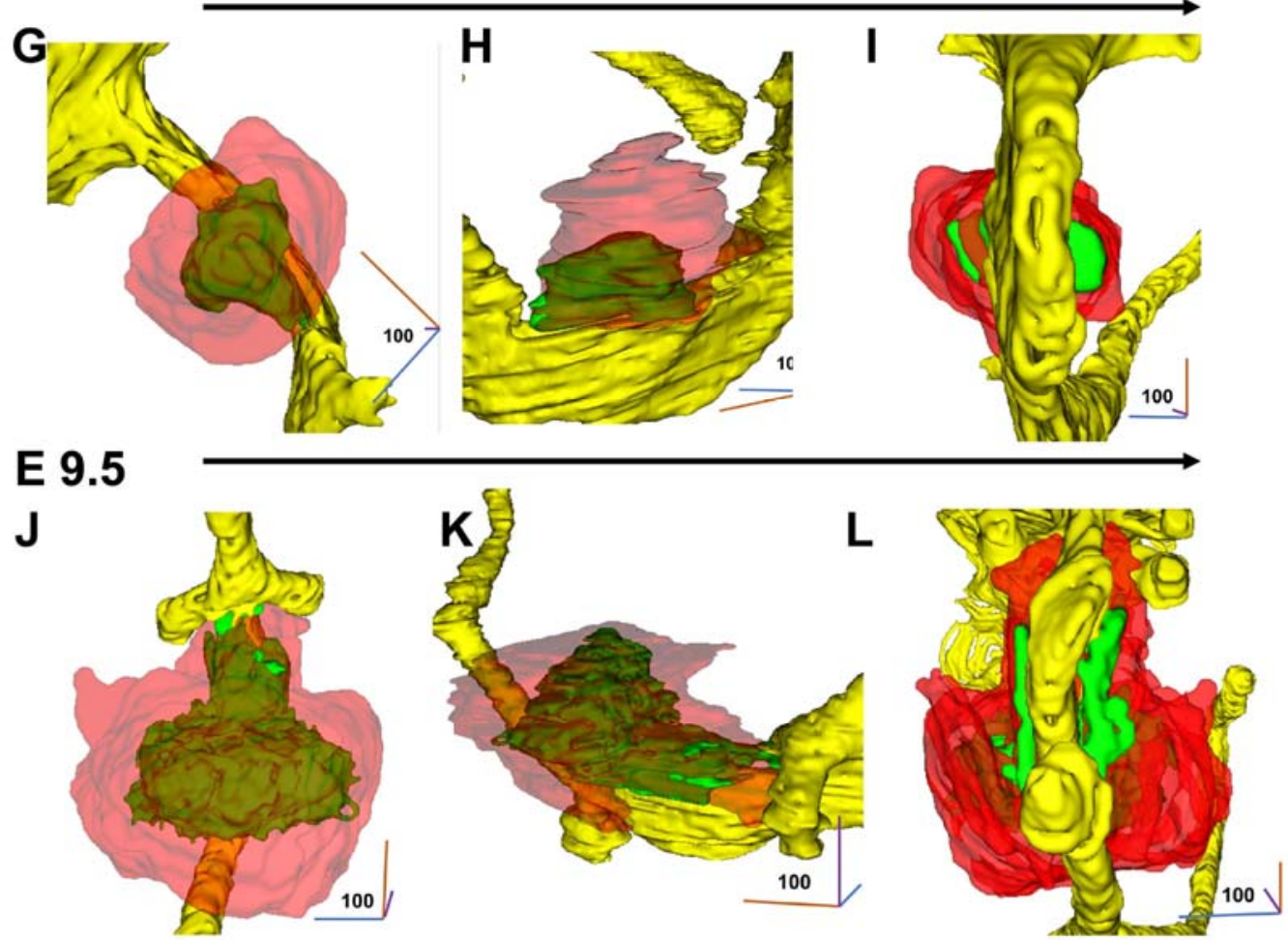

E 10.0 


\section{Figure 5. Visualization of 3D reconstructed murine liver bud and septum transversum mesenchyme (STM) interactions.}

The STM has a key role in liver development. To visualize liver bud-STM interactions during early liver bud morphogenesis, the liver bud, the gut tube, and the STM were visualized together during early liver bud development. This was performed as described in Figure 1. Each time point based on one specimen.

A) 3D reconstruction of the liver murine liver bud and STM, at E8.5. Ventral view. Liver bud (green), STM (red). Visualization of the STM at E8.5 demonstrates that it covers the inferior $\sim 1 / 2$ of the E8.5 liver bud.

B) Same as A) except right lateral view. The STM extends ventrally for a distance similar to the dorsalventral thickness of the liver bud.

C) Same as A) except inferior view. The width of the STM in the lateral direction is about $3 / 4$ of the lateral thickness of the liver bud.

D) 3D reconstruction of the liver murine liver bud and STM, at E9.0 Superior, ventral, lateral view. the STM extends laterally for a length equal to $3 / 4$ times the lateral (smaller) diameter, and covers approximately $80 \%$ of the liver bud.

E) Same as D) except ventral, right lateral view, demonstrating the STM extends laterally for a length equal to $3 / 4$ times the lateral (smaller) diameter, and covers approximately $80 \%$ of the liver bud.

F) Same as E) except ventral, inferior (anterior) view. This demonstrates how the STM contacts the liver bud directly.

G) 3D reconstruction of the liver murine liver bud and septum transversum mesenchyme, at E9.5 Ventral, lateral view. The STM extends laterally about $1 / 2$ times the diameter of the liver bud, and fully surrounds the liver bud. This suggests that the STM grows, remodels with the liver bud, and potentially primes the liver for growth.

H) Same as G) except right lateral view. The STM appears to be as thick as the liver bud when viewed laterally.

I) Same as G) except dorsal view. The STM extends laterally, for a distance of $1 / 3$ times the lateral width of the liver bud.

J) 3D reconstruction of the liver murine liver bud and STM, at E10.0 Ventral, lateral view. Compared to E9.5, the lateral STM thickness has increased.

K) Same as J) except left lateral view. The STM has diminished in the ventral direction.

L) Same as J) except posterior view. The STM has diminished in the lateral direction. 

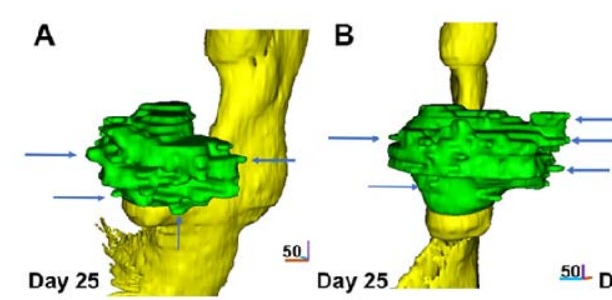

C
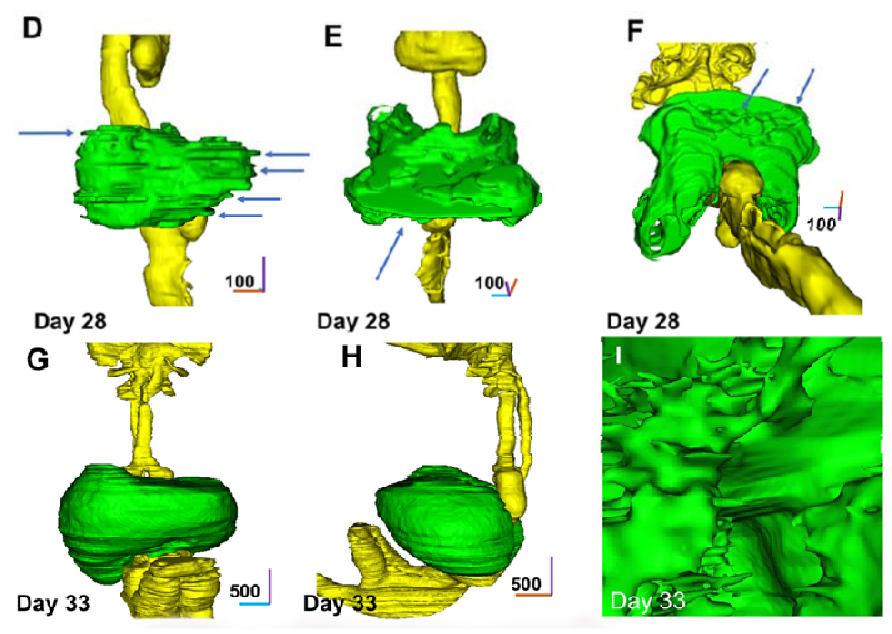

J

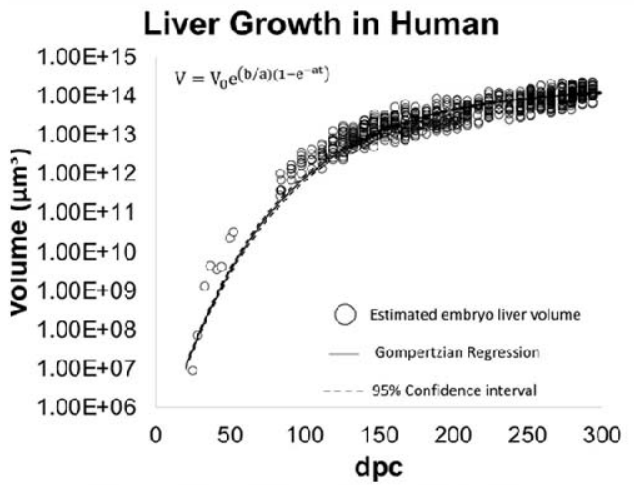

$\mathbf{K}$

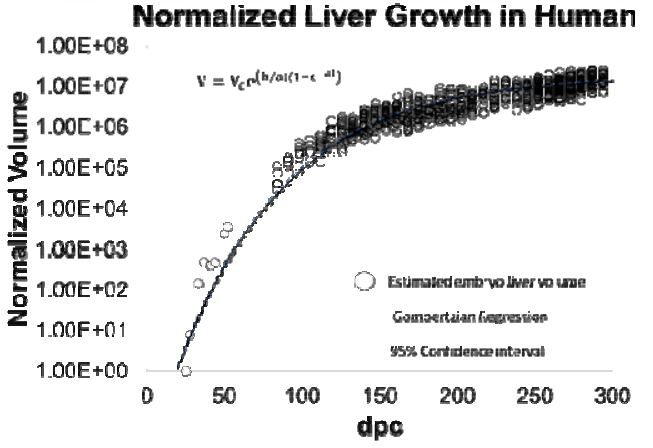

Figure 6. 3D reconstruction and visualization of the developing human liver bud.

To understand dynamics of liver bud morphogenesis, the human liver bud was imaged in 3D similar to the procedure used to visualize the mouse liver bud. 3D datasets, consisting of cleared hematoxylin and eosin (H\&E) tissue sections from the 3D Atlas of Human Embryology were used. Datasets were reformatted, imported, segmented, and reconstructed in 3D. Ventral views are shown. Each time point based on one specimen. Gut tube (yellow), the liver bud (green). Sample per timepoint are $n=9$, Days 25$56, \mathrm{n}=930$, Day $125-294$
A) 3D reconstruction of the human liver bud on day 25. Arrows demonstrate hepatic cords (arrows), and sheet-like growth appears
B) Same as A) except ventral view. Lateral hepatic cords (arrows) can be visualized, and sheet-like growth is again observed.
C) Same as A) except, inferior, left lateral, ventral view. Lateral hepatic cords can be visualized (arrows), and inferior view enables observation of sheet like growth. In some cases, sheets of cells are bounded by hepatic cords. Layers of sheets can be clearly visualized.
D) 3D reconstruction focused of the human liver bud on day 28. Lateral view. Arrows depict migrating cords. Although larger than day 25, the liver bud demonstrates sheet-like growth and hepatic cords.
E) Same as D) except anterior, dorsal view. Arrow depicts tissue which wraps around anatomical structures. Dorsal side does not demonstrate appreciable hepatic cords.
F) Same as D) except ventral view. Arrows depicting sheet of migrating cells extending medially and laterally.
G) 3D reconstruction focused of the human liver bud on day 33. Ventral view. Image demonstrates that the liver has enlarged and is smoothened, with no hepatic cords or cell sheets are present.
H) Same as G) except lateral view. Again, no hepatic cords or hepatic sheets are present. Inside view of the 3D reconstruction focused of the human liver bud on day 33 .
I) The green images demonstrate numerous enclosed green structures and trabeculation, all of which are in fact empty spaces within the 3D constructed liver. Interconnections can be seen inside the liver as the green portions are enclosed voids.


J) Absolute human liver volume changes are modelled using a non-linear regression Gompertzian model of tissue growth and fitted to the equation $V=V_{o} e^{\frac{b}{a} *\left(1-e^{-a t}\right)}$. Assuming $95 \%$ confidence interval, whereby $V_{o}=50,000, \mathrm{a}=0.014,0.015 ; \mathrm{b}=0.31,0.33 ; \mathrm{P}<0.001, \mathrm{R}^{2}=0.84$ and RMSE $=$ $3.06 \times 10^{6}$

K) Relative human liver volume was calculated by normalizing to the embryo liver volume at day 25. Changes are modeled using a non-linear regression Gompertzian model of tissue growth and fitted to the equation $V=V_{o} e^{\frac{b}{a} *\left(1-e^{-a t}\right)}$. Assuming 95\% confidence interval, whereby $V_{o}=0.0056$, $\mathrm{a}=0.014,0.015 ; \mathrm{b}=0.31,0.33 ; \mathrm{P}<0.001, \mathrm{R}^{2}=0.84$ and $\mathrm{RMSE}=1.04 \times 10^{3}$ 


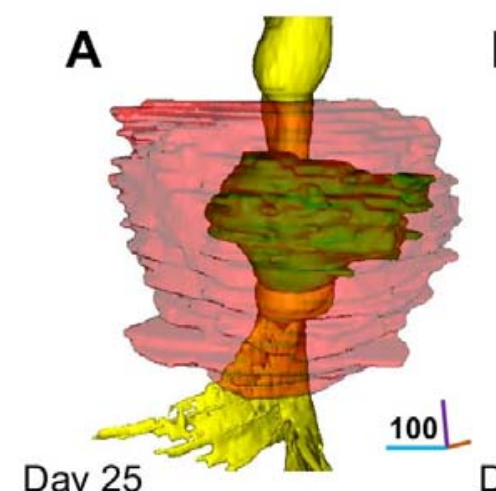

B
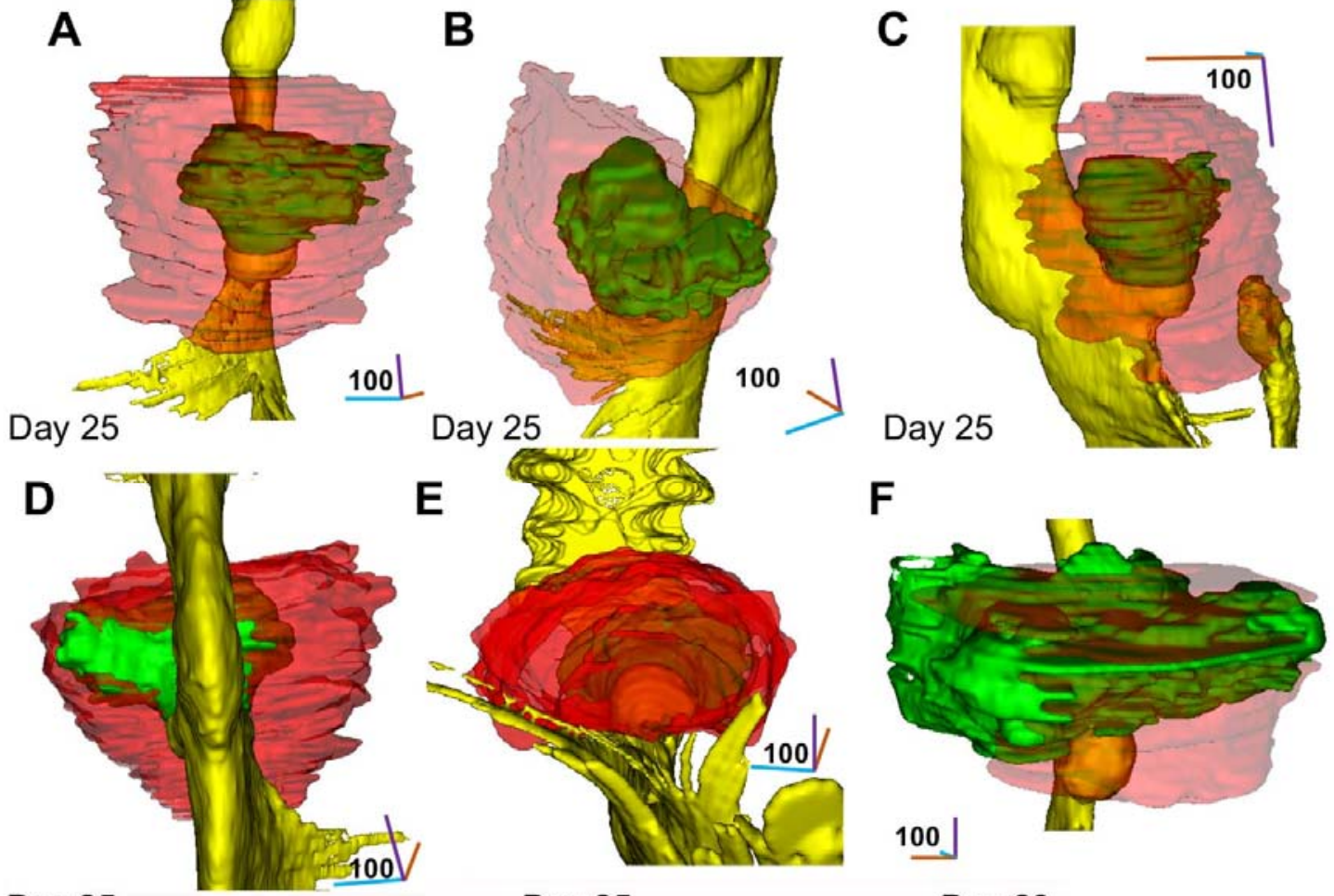

Day 25

Day 25

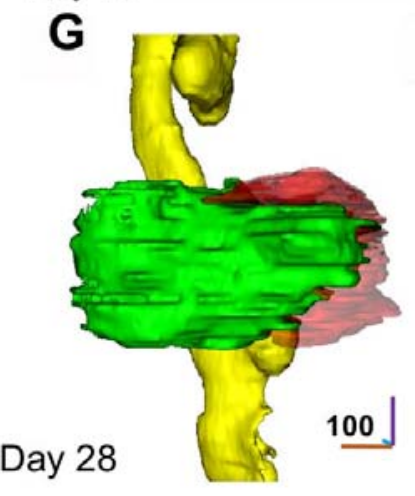

H

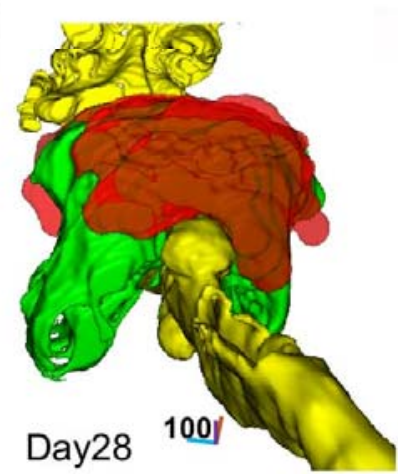

Day 28

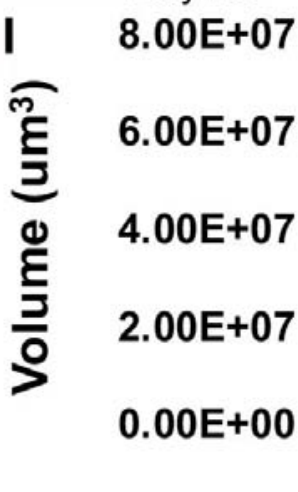

a STM " Liver 
B) Same as A, except superior, lateral view. The STM continues to envelop the liver in all directions.

C) Same as A, except right, lateral view. The STM envelops the liver.

D) Same as D, except dorsal view. Portions of the liver bud are free from STM, in the posterior portion of liver bud.

E) Same as A, except inferior view. STM envelops the liver bud, and appears to form a tail-like structure inferiorly.

F) 3D reconstruction of the human liver bud and STM, at Day 28. Ventral, lateral view. The STM is observable superiorly and inferiorly, but within the sheet of cells within the center of the liver.

G) Same as F, except right, lateral view. STM is not observed at this stage.

H) Same as F, except inferior view with the ventral surface facing up. STM is not observed on the dorsal side of liver but is present on the ventral side.

I) Quantification of human STM and liver bud volume during human liver development on days 25 and 28 . The liver growth, in terms of volume, has overtaken the STM by day 28 , unlike the mouse. 

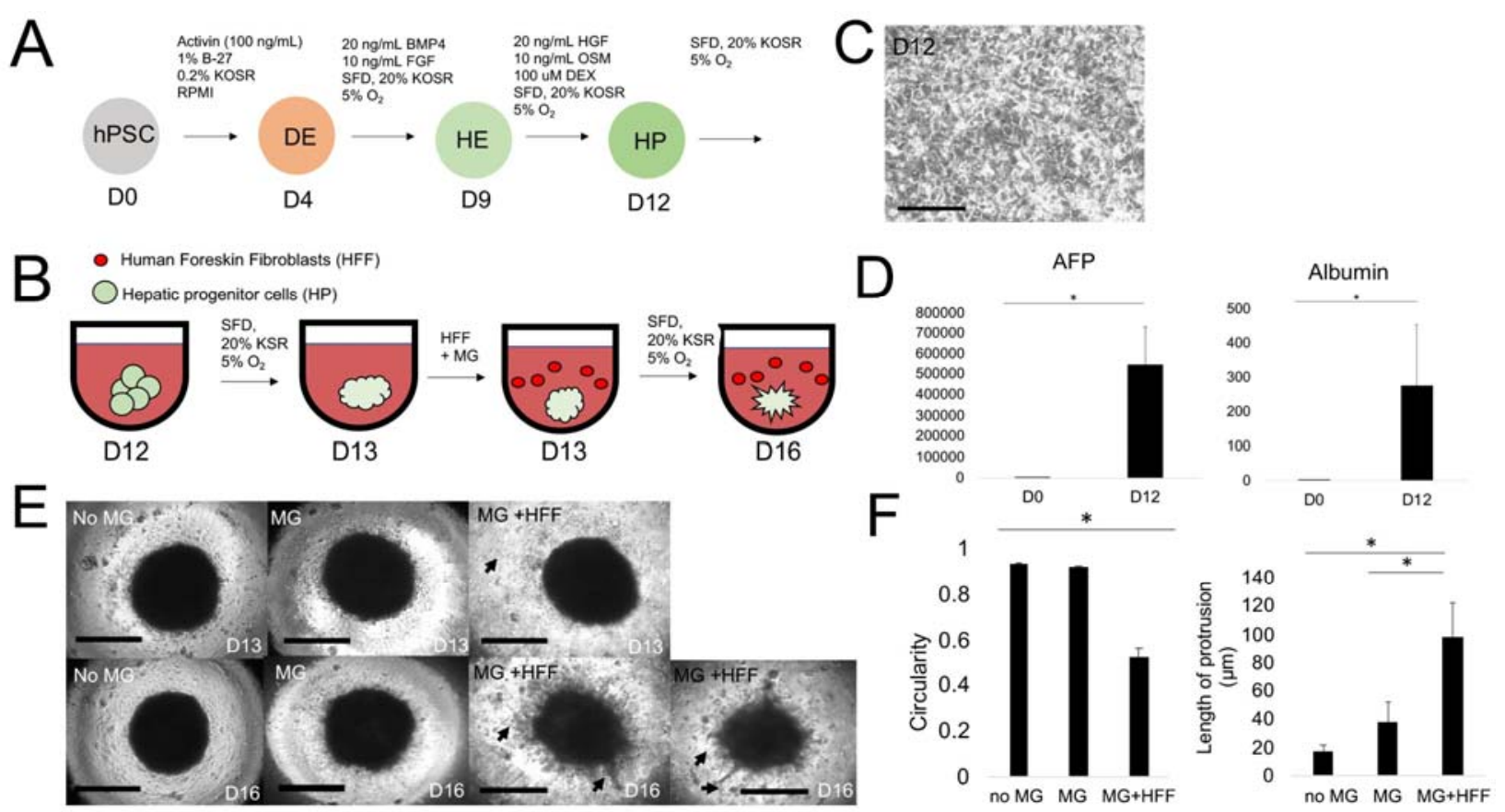

Control

Experimental

Figure 8. Epithelial-mesenchymal interactions in an human stem cell-derived organoid model of human liver bud.

A) hPSC derived hepatic progenitor cells are differentiated using our illustrated scheme in from hPSCs to definitive endoderm (DE). Subsequently cells are directed towards hepatic lineage using BPM4 (20 ng/mL) and FGF $(10 \mathrm{ng} / \mathrm{mL})$ to form hepatic endoderm (HE). Cells are then exposed to Hepatocyte growth factor (HGF) - $20 \mathrm{ng} / \mathrm{ml}$, Dexamethasone (DEX) - $100 \mu \mathrm{M}$, and Oncostatin M (OSM) - $10 \mathrm{ng} / \mathrm{ml}$, for brief hepatic lineage maturation for form hepatic progenitors (HP). Medium is a nutrient rich medium (SFD) with $20 \%$ Knockout serum(defined serum), and experiments at under hypoxic conditions.

B) Schematic of 3D organoid formation using hPSC hepatic progenitor cells.

C) Morphology of 2D hepatic progenitor cells after Day 12 induction, Scale bar $=200 \mu \mathrm{m}$. Compact organoids form in 24 hours and can be subsequently embedded in Matrigel containing fibroblasts.

D) qRT-PCR analysis of Day 12 hepatic progenitor cells demonstrating significant increases in alpha-fetoprotein (AFP) and Albumin (ALB) expression. Plotted is relative fold change normalized to GAPDH, (AFP, day 0 vs day 12, $\mathrm{n}=3, \mathrm{P}=0.0069$ ); (Albumin, Day 0 vs Day 12, $\mathrm{n}=3, \mathrm{P}=0.054)$.

E) Images of hepatic organoids modeling liver bud in suspension culture, matrigel embedded culture, and Matrigel (MG) + human foreskin fibroblasts (HFF) embedded culture. There was significant migration in the MG+ HFF culture condition as compared to the no MG and MG no HFF from D13-D16. Scale bar $=300 \mu \mathrm{m}$.

F) Analysis of liver bud organoid 3D culture, there is significantly less circularity of the central organoid of liver bud model in the Matrigel+fibroblast condition ( $n=3, P=0.0033$ ). Subsequent characterization of the length of the protrusions from the central organoid demonstrated significant increases in the Matrigel+fibroblast culture condition over controls (MG+HFF vs. 3D sus, $n=3, P=0.0081)$; (MG+HFF vs. MG, $n=3, P=0.009)$. Plotted is mean \pm SD. Significance defined as $\mathrm{P} \leq 0.05$. 


\section{SUPPLEMENTARY FIGURE LEGENDS}

3 Supplemental Figure 1. Segmentation process of murine liver development in whole embryo at 4 E8.5.

Supplemental Figure 2. Dynamic 3D imaging of murine liver development in whole embryo.

A) Coronal, black and white, hematoxylin and eosin (H+E) stain tissue section at E9.0 of mouse development, obtained from eMouseAtlas database, and corresponding segmented image. In segmented image, the gut tube (yellow), the liver bud (green) and STM (red) were segmented. A = Anterior, $\mathrm{V}=$ Ventral, $\mathrm{P}=$ Posterior, $\mathrm{D}=$ Dorsal. Arrows depict liver bud.

B) Same as A, except sagittal sections and corresponding segmented image.

Supplemental Figure 3. Segmentation process of murine liver development in whole embryo at

E9.0.

Same as Supplemental Figure 2 except E10.0. Scale bar is in microns.

Supplemental Figure 4. Segmentation process of murine liver development in whole embryo at E10.0.

Supplemental Figure 5. Quantification of septum transversum mesenchyme (STM) volume during murine liver development.

To measure the dynamics of STM growth, volumes were measured after loading segmented images of embryos. Relative volumes were normalized by taking the ratio to the corresponding embryo volumes. Each time point based on one specimen.

A) Ratio of the reconstructed 3D liver murine liver growth compared to the mouse embryo at E8.5E10. STM growth mirrors the growth patterns of the liver bud.

B) Absolute volume of the reconstructed 3D liver murine liver growth at E8.5-E10. STM does not stay constant, but exhibits growth that patterns growth of the liver bud, including discontinuous growth. 
2 Supplemental Figure 6. 3D reconstruction and visualization of human liver development in whole 3 embryo.

A) Whole human embryo 3D reconstruction demonstrating reconstructed human embryo on Day 25.

Each tissue of interest was identified and thresholded and traced on individual, transverse tissue

(1)

(1)
slices using known cross sectional anatomy and pre-identified labels within the database. Gut tube (yellow), the liver bud (green) and STM (red). A = Anterior, V = Ventral, P = Posterior, D= Dorsal.

B) Same as A except Day 28 in human development.

12 Supplemental Figure 7. Ventral view of human liver development at Day 33.

The liver bud (green) and gut tube (yellow) is viewed at the anterior position looking towards

Supplemental Figure 8. Dorsal view of human liver development at Day 33.

Dorsal view of liver bud (green) and gut tube (yellow). Scale in microns.

Supplemental Video 1. Murine liver bud at E8.5 in $360^{\circ}$ rotation through sagittal plane.

Ventral view of liver bud (green) rotating through sagittal plane. Units in axis are microns.

Supplemental Video 2. Murine liver bud at E9.0 in $360^{\circ}$ rotation through sagittal plane.

Supplemental Video 3. Murine liver bud at E9.5 in $360^{\circ}$ rotation through sagittal plane.

Ventral view of liver bud (green) rotating through sagittal plane. Units in axis are microns.

Supplemental Video 4. Murine liver bud at $\mathbf{E 1 0 . 0}$ in $360^{\circ}$ rotation through sagittal plane. Ventral view of liver bud (green) rotating through sagittal plane. Units in axis are microns. 
Interior view of liver bud (green) through sagittal plane. Interior view is inverted as the structures (green) are voids within the liver bud and empty space is where the liver bud is solid. Units in axis are microns.

Supplemental Video 6. Murine liver bud and STM at E8.5 in $360^{\circ}$ rotation through sagittal plane. Ventral view of liver bud (green) and STM (red) rotating through sagittal plane. Units in axis are microns.

Supplemental Video 7. Murine liver bud and STM at E9.0 in $360^{\circ}$ rotation through sagittal plane.

Supplemental Video 8. Murine liver bud and STM at E9.5 in $360^{\circ}$ rotation through sagittal plane. Ventral view of liver bud (green) and STM (red) rotating through sagittal plane. Units in axis are microns.

Supplemental Video 9. Murine liver bud and STM at E10.0 in $360^{\circ}$ rotation through sagittal plane. Ventral view of liver bud (green) and STM (red) rotating through sagittal plane. Units in axis are

Supplemental Video 10. Human liver bud at Day 25 in $360^{\circ}$ rotation through sagittal plane.

Supplemental Video 11. Human liver bud at Day 25 in $360^{\circ}$ rotation through sagittal plane.

Supplemental Video 12. Human liver bud at Day 28 in $360^{\circ}$ rotation through sagittal plane. Ventral view of liver bud (green) rotating through sagittal plane. Units in axis are microns.

Supplemental Video 13. Human liver bud at Day 28 in $360^{\circ}$ rotation through sagittal plane. Ventral view of liver bud (green) rotating through transverse plane. Units in axis are microns.

Supplemental Video 14. Human liver bud at Day 33 in $360^{\circ}$ rotation through sagittal plane. Ventral view of liver bud (green) rotating through sagittal plane. Units in axis are microns. 
Supplemental Video. 15. Human liver bud at Day 33 in $360^{\circ}$ rotation through sagittal plane. Ventral view of liver bud (green) rotating through transverse plane. Units in axis are microns.

Supplemental Video 16. Interior view of human liver bud at day 33. Interior view of liver bud (green) through sagittal plane. Interior view is inverted as the structures (green) are voids within the liver bud and empty space is where the liver bud is solid. Units in

Supplemental Video 17. Human liver bud and STM at Day 25 in $360^{\circ}$ rotation through sagittal 11 plane. axis are microns.

Ventral view of liver bud (green) and STM (red) rotating through sagittal plane. Units in axis are

Supplemental Video 18. Human liver bud and STM at Day 25 in $360^{\circ}$ rotation through sagittal plane. microns.

Supplemental Video 19. Human liver bud and STM at Day 28 in $360^{\circ}$ rotation through sagittal plane.

Ventral view of liver bud (green) and STM (red) rotating through sagittal plane. Units in axis are

\section{Supplemental Video 20. Human liver bud and STM at Day 28 in $360^{\circ}$ rotation through sagittal} plane.

31 Not applicable.

\section{CONSENT FOR PUBLICATION}

34 Not applicable. 


\section{AVAILABILITY OF DATA AND MATERIALS}

3 All data generated or analyzed during this study are included in this published article and its

4 supplementary files.

COMPETING INTERESTS

7 The authors declare that they have no competing interests

FUNDING

NP was supported by University at Buffalo, School of Engineering, State University of NY startup funds and University at Buffalo, State University of NY, Stem cells and Regenerative Medicine program (ScIRM)", (New York Stem Cell Science NYSTEM (Contract \#C30290GG). OO was supported by the Western New York Prosperity Fellowship.

\section{AUTHOR CONTRIBUTIONS:}

16 OO: Obtained data, analyzed data, developed methodology for acquisition and analysis, wrote and 17 approved manuscript.

18 DG: Obtained data, analyzed data, developed methodology for acquisition and analysis, wrote and

19 approved manuscript.

20 TM: Obtained data, analyzed data, developed methodology for acquisition and analysis, wrote and

21 approved manuscript.

22 CS: Obtained data, analyzed data, approved manuscript

23 SR: Obtained data, analyzed data, approved manuscript

24 SR: Obtained data, analyzed data, approved manuscript

25 NP: Conceptualized, acquired funding, investigated, supervised, wrote, edited manuscript, and approved 26 manuscript.

\section{ACKNOWLEDGEMENTS:}

28 We thank Dr. Duncan Davidson and Professor Richard Baldock for their efforts in developing the eMouseAtlas Project which provided the datasets we used to perform our embryological analysis. We thank Professor R. Mark Henkelman, Dr. Jason P. Lerch, Dr. Brian J. Nieman, and Dr. John G. Sled for their work as part of the Mouse Imaging Centre and for providing us with additional image data sets for mouse development. We also like to thank Professor Bernadette S. de Bakker and the rest of the 3D Atlas team for providing us with human embryo data sets.

\section{REFERENCES}

36 Ayres-Silva Jde, P., P. P. Manso, M. R. Madeira, M. Pelajo-Machado and H. L. Lenzi (2011).

37 "Sequential morphological characteristics of murine fetal liver hematopoietic microenvironment

38 in Swiss Webster mice." Cell Tissue Res 344(3): 455-469. 
Baldock, R., J. Bard, M. Kaufman and D. Davidson (1992). "A real mouse for your computer." Bioessays 14(7): 501-502.

Bin, W. T., L. M. Ma, Q. Xu and X. L. Shi (2012). "Embryonic hepatocyte transplantation for hepatic cirrhosis: efficacy and mechanism of action." World J Gastroenterol 18(4): 309-322. Bort, R., M. Signore, K. Tremblay, J. P. Martinez Barbera and K. S. Zaret (2006). "Hex homeobox gene controls the transition of the endoderm to a pseudostratified, cell emergent epithelium for liver bud development." Dev Biol 290(1): 44-56.

Boylan, J. M., H. Francois-Vaughan, P. A. Gruppuso and J. A. Sanders (2017). "Engraftment and Repopulation Potential of Late Gestation Fetal Rat Hepatocytes." Transplantation 101(10): 23492359.

Cascio, S. and K. S. Zaret (1991). "Hepatocyte differentiation initiates during endodermalmesenchymal interactions prior to liver formation." Development 113(1): 217-225. Cayuso, J., A. Dzementsei, J. C. Fischer, G. Karemore, S. Caviglia, J. Bartholdson, G. J. Wright and E. A. Ober (2016). "EphrinB1/EphB3b Coordinate Bidirectional Epithelial-Mesenchymal Interactions Controlling Liver Morphogenesis and Laterality." Dev Cell 39(3): 316-328. Chen, C., A. Soto-Gutierrez, P. M. Baptista and B. Spee (2018). "Biotechnology Challenges to In Vitro Maturation of Hepatic Stem Cells." Gastroenterology 154(5): 1258-1272. Chen, F., P. W. Tillberg and E. S. Boyden (2015). "Optical imaging. Expansion microscopy." Science 347(6221): 543-548. Collardeau-Frachon, S. and J. Y. Scoazec (2008). "Vascular development and differentiation during human liver organogenesis." Anat Rec (Hoboken) 291(6): 614-627. Dabeva, M. D., P. M. Petkov, J. Sandhu, R. Oren, E. Laconi, E. Hurston and D. A. Shafritz (2000). "Proliferation and differentiation of fetal liver epithelial progenitor cells after transplantation into adult rat liver." Am J Pathol 156(6): 2017-2031. Darwiche, H. and B. E. Petersen (2010). "Biology of the adult hepatic progenitor cell: "ghosts in the machine"." Prog Mol Biol Transl Sci 97: 229-249. de Bakker, B. S., K. H. de Jong, J. Hagoort, K. de Bree, C. T. Besselink, F. E. de Kanter, T. Veldhuis, B. Bais, R. Schildmeijer, J. M. Ruijter, R. J. Oostra, V. M. Christoffels and A. F. Moorman (2016). "An interactive three-dimensional digital atlas and quantitative database of human development." Science 354(6315).

de Bakker, B. S., K. H. de Jong, J. Hagoort, R. J. Oostra and A. F. Moorman (2012). "Towards a 3-dimensional atlas of the developing human embryo: the Amsterdam experience." Reprod Toxicol 34(2): 225-236.

Ema, H. and H. Nakauchi (2000). "Expansion of hematopoietic stem cells in the developing liver of a mouse embryo." Blood 95(7): 2284-2288.

Enzan, H., H. Himeno, M. Hiroi, H. Kiyoku, T. Saibara and S. Onishi (1997). "Development of hepatic sinusoidal structure with special reference to the Ito cells." Microsc Res Tech 39(4): 336349.

Gordillo, M., T. Evans and V. Gouon-Evans (2015). "Orchestrating liver development."

40 Development 142(12): 2094-2108.

41 Hussain, S. Z., T. Sneddon, X. Tan, A. Micsenyi, G. K. Michalopoulos and S. P. Monga (2004). 157-169.

44 Kamei, Y., O. Tsutsumi, A. Yamakawa, Y. Oka, Y. Taketani and J. Imaki (1999). "Maternal 45 Epidermal Growth Factor Deficiency Causes Fetal Hypoglycemia and Intrauterine Growth 
Retardation in Mice: Possible Involvement of Placental Glucose Transporter GLUT3 Expression1." Endocrinology 140(9): 4236-4243.

Kamiya, A., T. Kinoshita, Y. Ito, T. Matsui, Y. Morikawa, E. Senba, K. Nakashima, T. Taga, K. Yoshida, T. Kishimoto and A. Miyajima (1999). "Fetal liver development requires a paracrine action of oncostatin M through the gp130 signal transducer." EMBO J 18(8): 2127-2136. Koike, H., K. Iwasawa, R. Ouchi, M. Maezawa, K. Giesbrecht, N. Saiki, A. Ferguson, M. Kimura, W. L. Thompson, J. M. Wells, A. M. Zorn and T. Takebe (2019). "Modelling human hepato-biliary-pancreatic organogenesis from the foregut-midgut boundary." Nature 574(7776): 112-116.

Konstantinidis, D. G., K. M. Giger, M. Risinger, S. Pushkaran, P. Zhou, P. Dexheimer, S. Yerneni, P. Andreassen, U. Klingmuller, J. Palis, Y. Zheng and T. A. Kalfa (2015). "Cytokinesis failure in RhoA-deficient mouse erythroblasts involves actomyosin and midbody dysregulation and triggers p53 activation." Blood 126(12): 1473-1482. LeCouter, J., D. R. Moritz, B. Li, G. L. Phillips, X. H. Liang, H. P. Gerber, K. J. Hillan and N. Ferrara (2003). "Angiogenesis-independent endothelial protection of liver: role of VEGFR-1." Science 299(5608): 890-893. Ledouarin, N. (1963). "[Early Inductive Action of Mesoderm of the Cardiac Area on Hepatic Endoderm of the Chick Embryo]." C R Hebd Seances Acad Sci 257: 1357-1360. Lee, C. S., J. R. Friedman, J. T. Fulmer and K. H. Kaestner (2005). "The initiation of liver development is dependent on Foxa transcription factors." Nature 435(7044): 944-947. Lickert, H., J. K. Takeuchi, I. Von Both, J. R. Walls, F. McAuliffe, S. L. Adamson, R. M. Henkelman, J. L. Wrana, J. Rossant and B. G. Bruneau (2004). "Baf60c is essential for function of BAF chromatin remodelling complexes in heart development." Nature 432(7013): 107-112. Lints, T. J., L. Hartley, L. M. Parsons and R. P. Harvey (1996). "Mesoderm-specific expression of the divergent homeobox gene Hlx during murine embryogenesis." Dev Dyn 205(4): 457-470. Lotto, J., S. Drissler, R. Cullum, W. Wei, M. Setty, E. M. Bell, S. C. Boutet, S. Nowotschin, Y. Y. Kuo, V. Garg, D. Pe'er, D. M. Church, A. K. Hadjantonakis and P. A. Hoodless (2020). "Single-Cell Transcriptomics Reveals Early Emergence of Liver Parenchymal and Nonparenchymal Cell Lineages." Cell 183(3): 702-716 e714. Ludtke, T. H., V. M. Christoffels, M. Petry and A. Kispert (2009). "Tbx3 promotes liver bud expansion during mouse development by suppression of cholangiocyte differentiation." Hepatology 49(3): 969-978.

Man, J., J. C. Hutchinson, M. Ashworth, I. Jeffrey, A. E. Heazell and N. J. Sebire (2016). "Organ weights and ratios for postmortem identification of fetal growth restriction: utility and confounding factors." Ultrasound Obstet Gynecol 48(5): 585-590.

Margagliotti, S., F. Clotman, C. E. Pierreux, J. B. Beaudry, P. Jacquemin, G. G. Rousseau and F. P. Lemaigre (2007). "The Onecut transcription factors HNF-6/OC-1 and OC-2 regulate early liver expansion by controlling hepatoblast migration." Dev Biol 311(2): 579-589.

Margagliotti, S., F. Clotman, C. E. Pierreux, P. Lemoine, G. G. Rousseau, P. Henriet and F. P. Lemaigre (2008). "Role of metalloproteinases at the onset of liver development." Dev Growth Differ 50(5): 331-338.

42 Marguerat, S. and J. Bahler (2012). "Coordinating genome expression with cell size." Trends 43 Genet 28(11): 560-565.

44 Martinez Barbera, J. P., M. Clements, P. Thomas, T. Rodriguez, D. Meloy, D. Kioussis and R. S. 45 Beddington (2000). "The homeobox gene Hex is required in definitive endodermal tissues for 46 normal forebrain, liver and thyroid formation." Development 127(11): 2433-2445. 
Matsumoto, K., H. Yoshitomi, J. Rossant and K. S. Zaret (2001). "Liver organogenesis promoted by endothelial cells prior to vascular function." Science 294(5542): 559-563.

Mehrara, E., E. Forssell-Aronsson, V. Johanson, L. Kolby, R. Hultborn and P. Bernhardt (2013). "A new method to estimate parameters of the growth model for metastatic tumours." Theor Biol Med Model 10: 31 .

Nevzorova, Y. A., R. Tolba, C. Trautwein and C. Liedtke (2015). "Partial hepatectomy in mice." Lab Anim 49(1 Suppl): 81-88.

Nieman, B. J., M. D. Wong and R. M. Henkelman (2011). "Genes into geometry: imaging for mouse development in 3D." Curr Opin Genet Dev 21(5): 638-646.

Nomura, T. (1976). "Comparison of tumour susceptibility among various organs of foetal, young and adult ICR/Jcl mice." Br J Cancer 33(5): 521-534.

Ober, E. A. and F. P. Lemaigre (2018). "Development of the liver: Insights into organ and tissue morphogenesis." J Hepatol 68(5): 1049-1062. Ogoke, O., M. Maloy and N. Parashurama (2021). "The science and engineering of stem cellderived organoids-examples from hepatic, biliary, and pancreatic tissues." Biol Rev Camb Philos Soc 96(1): 179-204.

17 Ogoke, O., J. Oluwole and N. Parashurama (2017). "Bioengineering considerations in liver regenerative medicine." J Biol Eng 11: 46. Ogoke, O. Yousef. O. Ott. C. , Kalinousky. A., Lin. W., Shamul. C. Ross. S. Parashurama. N. (2020). "Mesenchyme modulates three-dimensional, collective cell migration of liver cells in vitro - a role for TGF $\beta$ pathway." Frontiers in Bioengineering and Biotechnology (Tissue Engineering) (In Press).

Overmoyer, B. A., C. E. McLaren and G. M. Brittenham (1987). "Uniformity of liver density and nonheme (storage) iron distribution." Arch Pathol Lab Med 111(6): 549-554. Pandey, E., A. S. Nour and E. N. Harris (2020). "Prominent Receptors of Liver Sinusoidal Endothelial Cells in Liver Homeostasis and Disease." Front Physiol 11: 873. Pardi, G. and I. Cetin (2006). "Human fetal growth and organ development: 50 years of discoveries." Am J Obstet Gynecol 194(4): 1088-1099.

Phoon, C. K. (2006). "Imaging tools for the developmental biologist: ultrasound biomicroscopy of mouse embryonic development." Pediatr Res 60(1): 14-21.

Richardson, L., S. Venkataraman, P. Stevenson, Y. Yang, J. Moss, L. Graham, N. Burton, B. Hill, J. Rao, R. A. Baldock and C. Armit (2014). "EMAGE mouse embryo spatial gene expression database: 2014 update." Nucleic Acids Res 42(Database issue): D835-844. Rossi, J. M., N. R. Dunn, B. L. Hogan and K. S. Zaret (2001). "Distinct mesodermal signals, including BMPs from the septum transversum mesenchyme, are required in combination for hepatogenesis from the endoderm." Genes Dev 15(15): 1998-2009.

37 Schmidt, C., F. Bladt, S. Goedecke, V. Brinkmann, W. Zschiesche, M. Sharpe, E. Gherardi and C. Birchmeier (1995). "Scatter factor/hepatocyte growth factor is essential for liver development." Nature 373(6516): 699-702.

Semeraro, R., V. Cardinale, G. Carpino, R. Gentile, C. Napoli, R. Venere, M. Gatto, R. Brunelli, E. Gaudio and D. Alvaro (2013). "The fetal liver as cell source for the regenerative medicine of liver and pancreas." Ann Transl Med 1(2): 13.

Sherwood, R. I., T. Y. Chen and D. A. Melton (2009). "Transcriptional dynamics of endodermal organ formation." Dev Dyn 238(1): 29-42.

Si-Tayeb, K., F. P. Lemaigre and S. A. Duncan (2010). "Organogenesis and development of the liver." Dev Cell 18(2): 175-189. 
Song, H., J. Luo, W. Luo, J. Weng, Z. Wang, B. Li, D. Li and M. Liu (2008). "Inactivation of Gprotein-coupled receptor 48 (Gpr48/Lgr4) impairs definitive erythropoiesis at midgestation through down-regulation of the ATF4 signaling pathway." J Biol Chem 283(52): 36687-36697. Sosa-Pineda, B., J. T. Wigle and G. Oliver (2000). "Hepatocyte migration during liver development requires Prox1." Nat Genet 25(3): 254-255.

Suzuki, T., Y. Kanai, T. Hara, J. Sasaki, T. Sasaki, M. Kohara, T. Maehama, C. Taya, H. Shitara, H. Yonekawa, M. A. Frohman, T. Yokozeki and Y. Kanaho (2006). "Crucial role of the small GTPase ARF6 in hepatic cord formation during liver development." Mol Cell Biol 26(16): 61496156.

Syed, S. H., K. V. Larin, M. E. Dickinson and I. V. Larina (2011). "Optical coherence tomography for high-resolution imaging of mouse development in utero." J Biomed Opt 16(4): 046004. Szpinda, M., M. Paruszewska-Achtel, A. Wozniak, C. Mila-Kierzenkowska, G. ElminowskaWenda, M. Dombek, A. Szpinda and M. Badura (2015). "Volumetric Growth of the Liver in the Human Fetus: An Anatomical, Hydrostatic, and Statistical Study." Biomed Res Int 2015: 858162.

17 Tanimizu, N. and T. Mitaka (2017). "Epithelial Morphogenesis during Liver Development." 18 Cold Spring Harb Perspect Biol 9(8).

Tatsumi, N., R. Miki, K. Katsu and Y. Yokouchi (2007). "Neurturin-GFRalpha2 signaling controls liver bud migration along the ductus venosus in the chick embryo." Dev Biol 307(1): 14-28.

Turnbull, D. H. and S. Mori (2007). "MRI in mouse developmental biology." NMR Biomed 20(3): 265-274.

"Quantitative imaging of cell dynamics in mouse embryos using light-sheet microscopy." Development 141(22): 4406-4414.

Watt, A. J., R. Zhao, J. Li and S. A. Duncan (2007). "Development of the mammalian liver and ventral pancreas is dependent on GATA4." BMC Dev Biol 7: 37.

Weinstein, M., S. P. Monga, Y. Liu, S. G. Brodie, Y. Tang, C. Li, L. Mishra and C. X. Deng (2001). "Smad proteins and hepatocyte growth factor control parallel regulatory pathways that converge on beta1-integrin to promote normal liver development." Mol Cell Biol 21(15): 51225131.

Wong, M. D., M. C. van Eede, S. Spring, S. Jevtic, J. C. Boughner, J. P. Lerch and R. M. Henkelman (2015). "4D atlas of the mouse embryo for precise morphological staging." Development 142(20): 3583-3591.

Xie, C., L. O. Schwen, W. Wei, A. Schenk, S. Zafarnia, F. Gremse and U. Dahmen (2016). "Quantification of Hepatic Vascular and Parenchymal Regeneration in Mice." PLoS One 11(8): e0160581. disturbance in fetal liver hematopoiesis of Mll-mutant mice." Blood 92(1): 108-117. Yang, D., P. Gao, C. Tian and Y. Sheng (2020). "Gompertz tracking of the growth trajectories of the human-liver-cancer xenograft-tumors in nude mice." Comput Methods Programs Biomed 191: 105412. 
1 Zhao, R., A. J. Watt, J. Li, J. Luebke-Wheeler, E. E. Morrisey and S. A. Duncan (2005).

2 "GATA6 is essential for embryonic development of the liver but dispensable for early heart

3 formation." Mol Cell Biol 25(7): 2622-2631.

4 Zorn, A. M. (2008). Liver development. StemBook. Cambridge (MA). 
A

Absolute Volumetric Liver Growth in Mouse

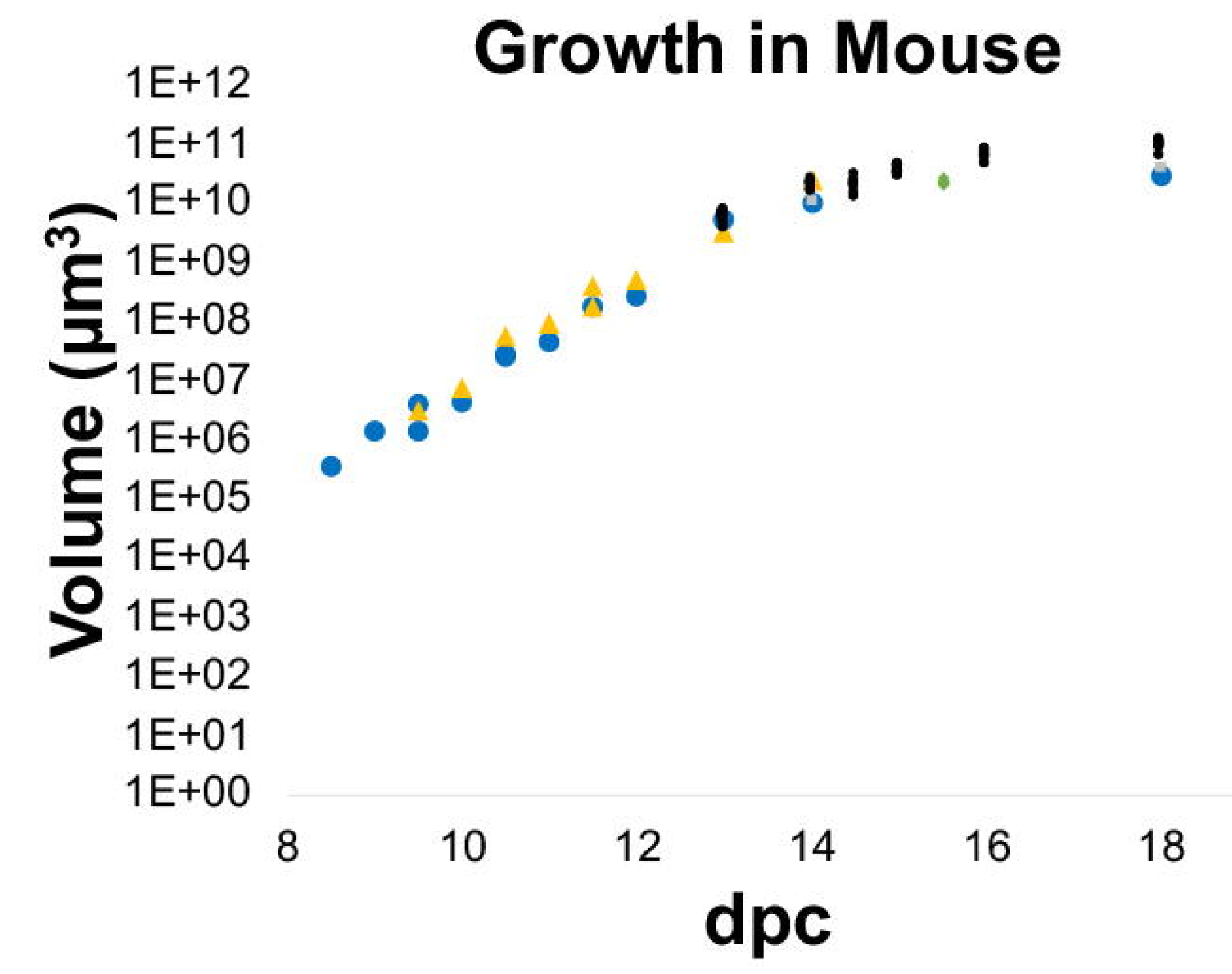

E

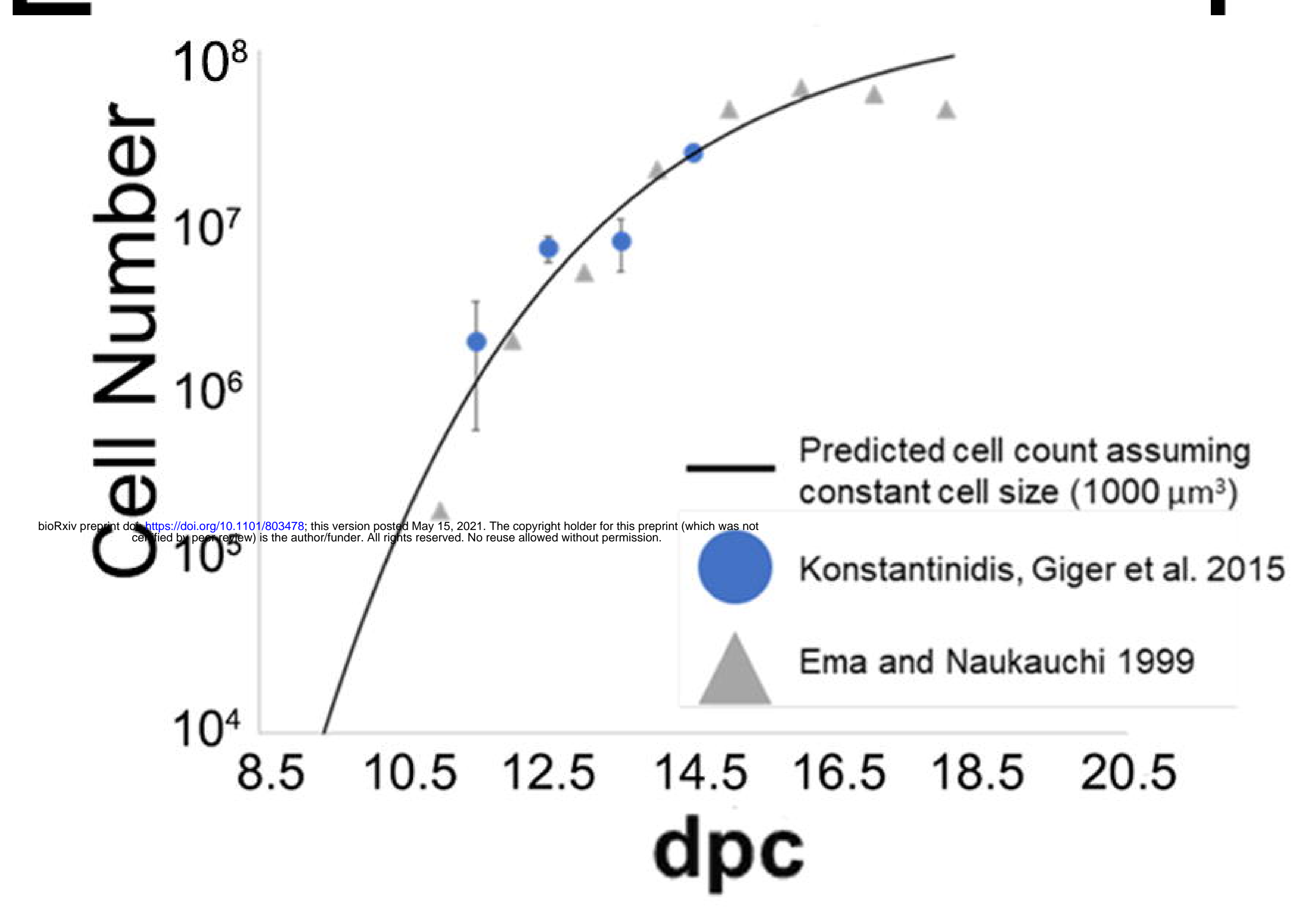

B Normalized Volumetric Liver

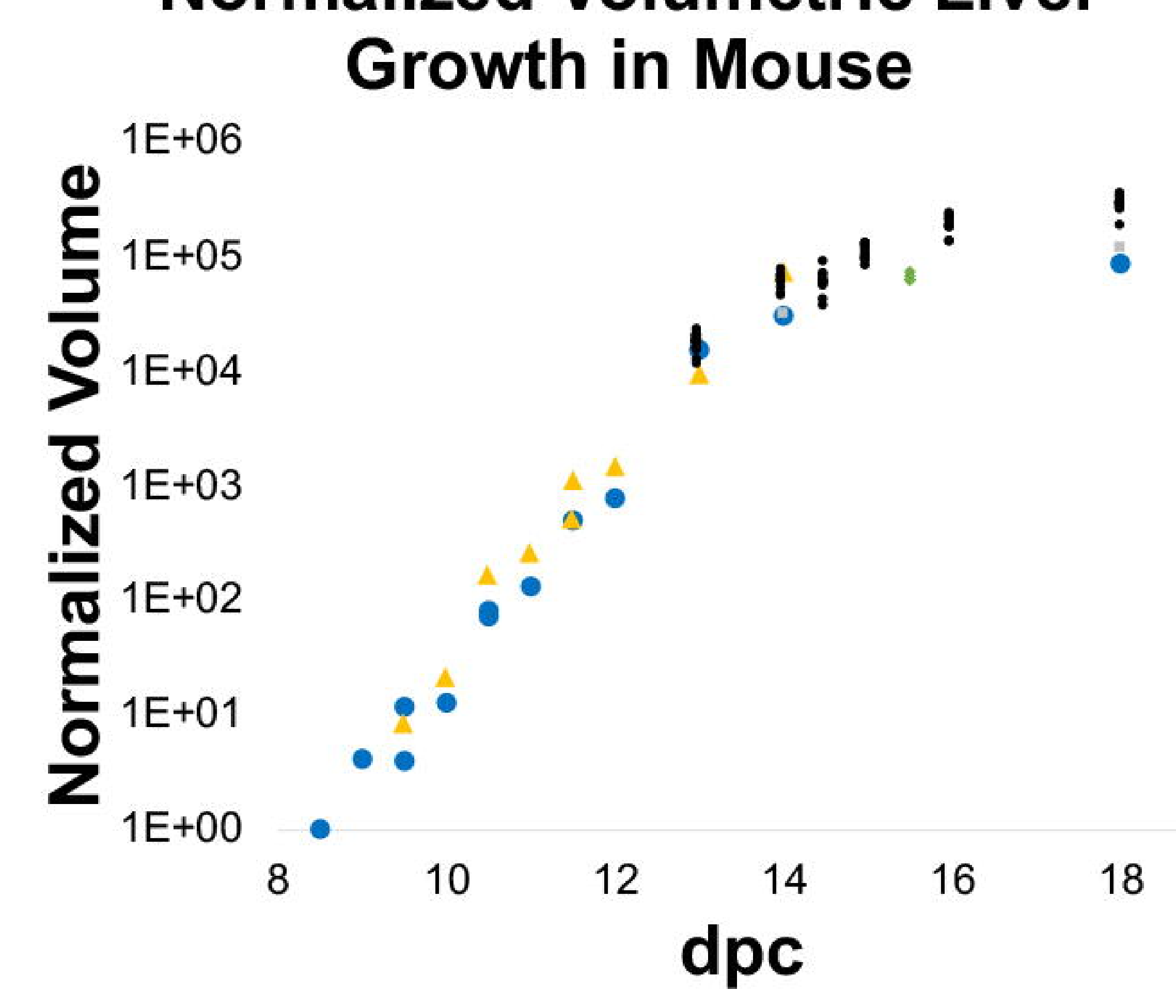

Modeling Absolute Volumetric Liver Growth in Mouse

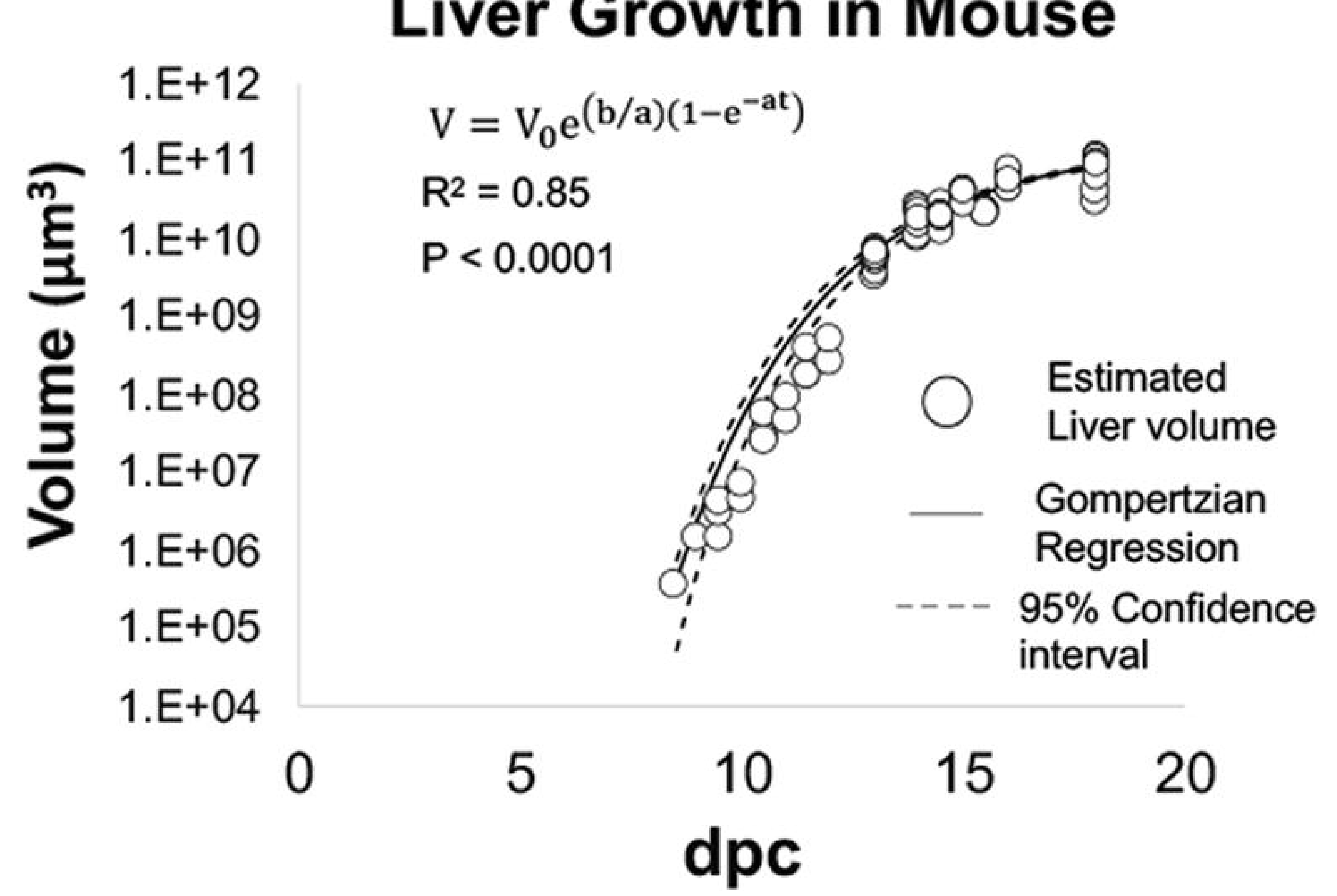

Modeling Normalized Volumetric Liver Growth

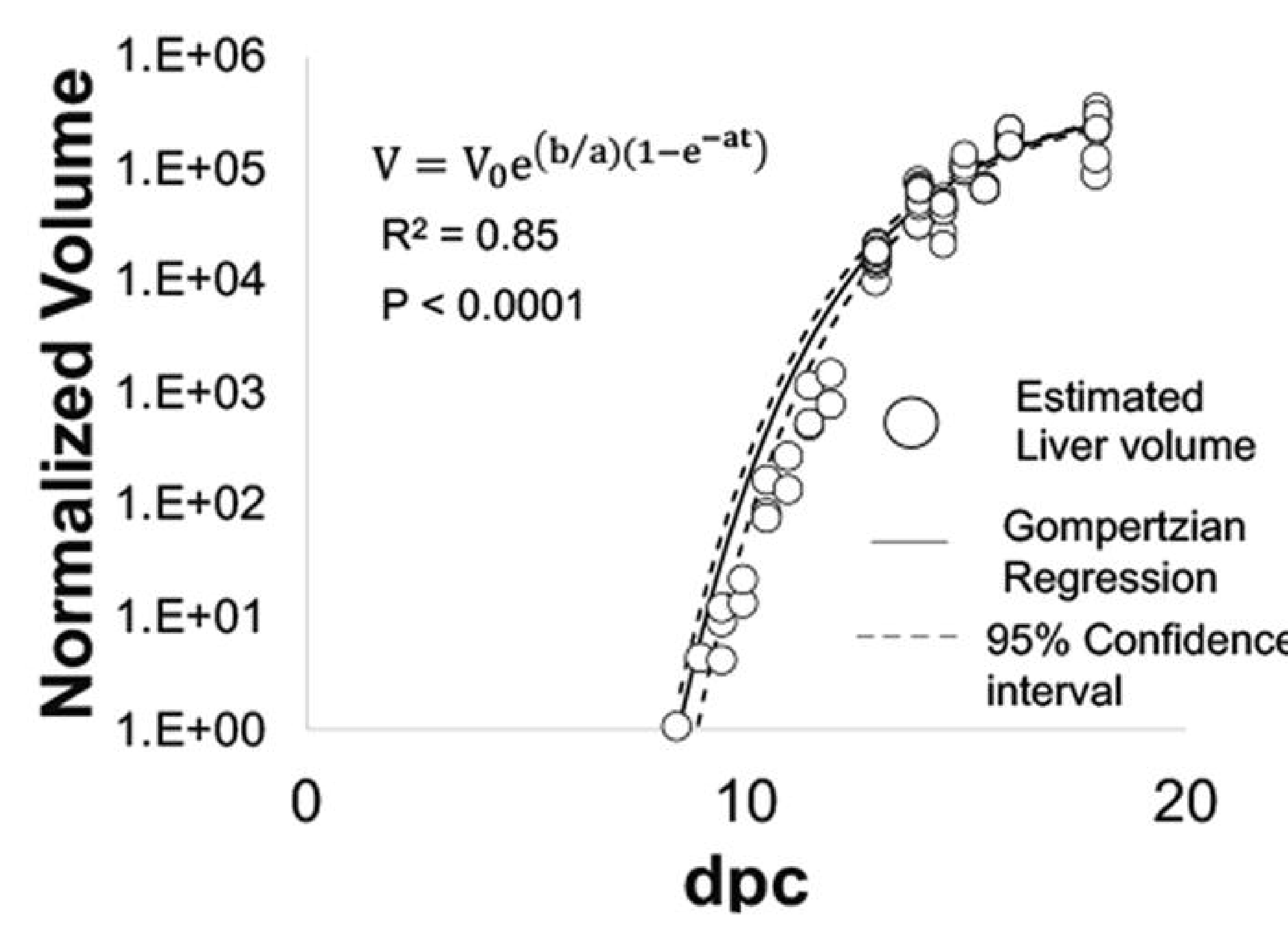

$\mathrm{H}$

E11.5
$F$

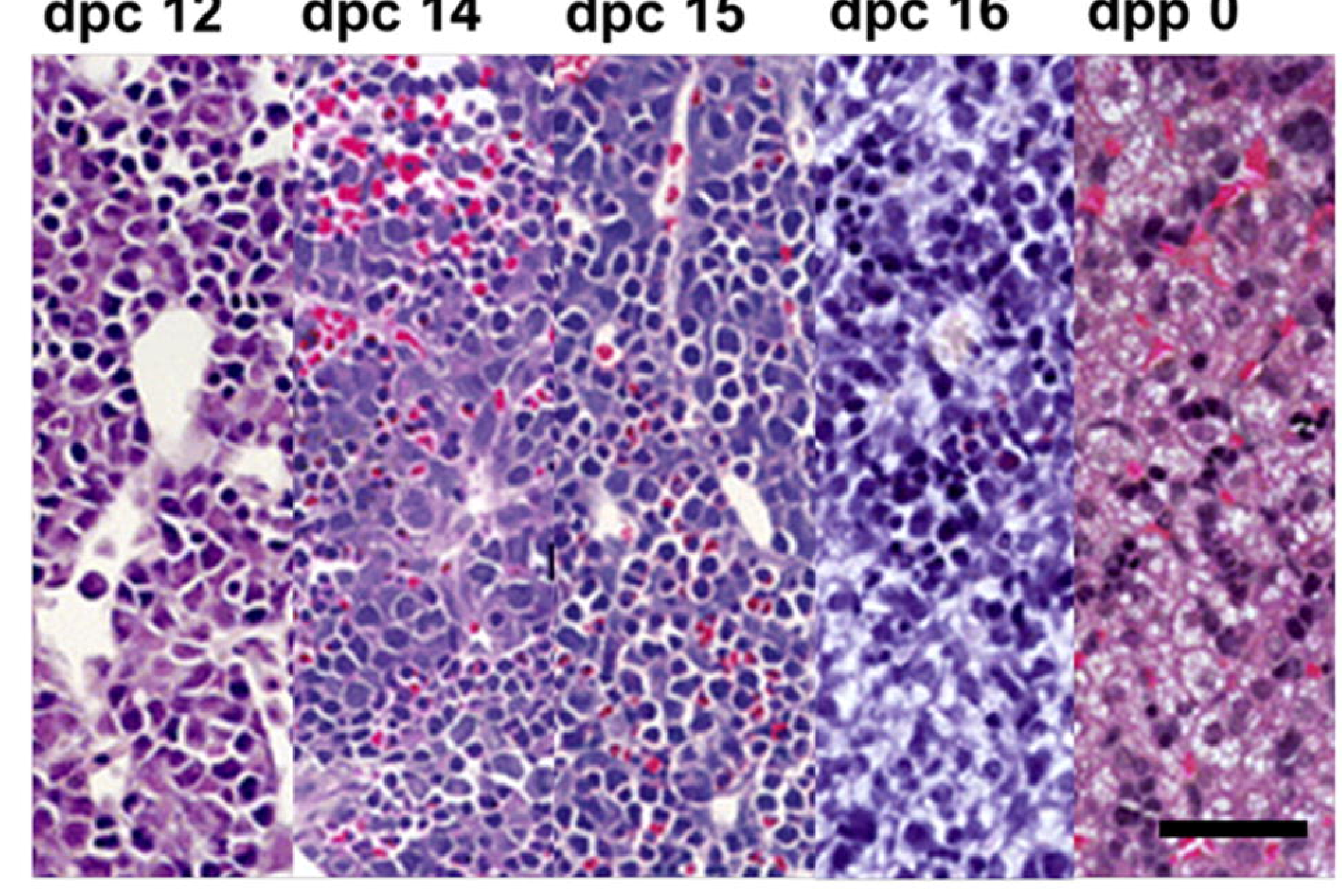

G

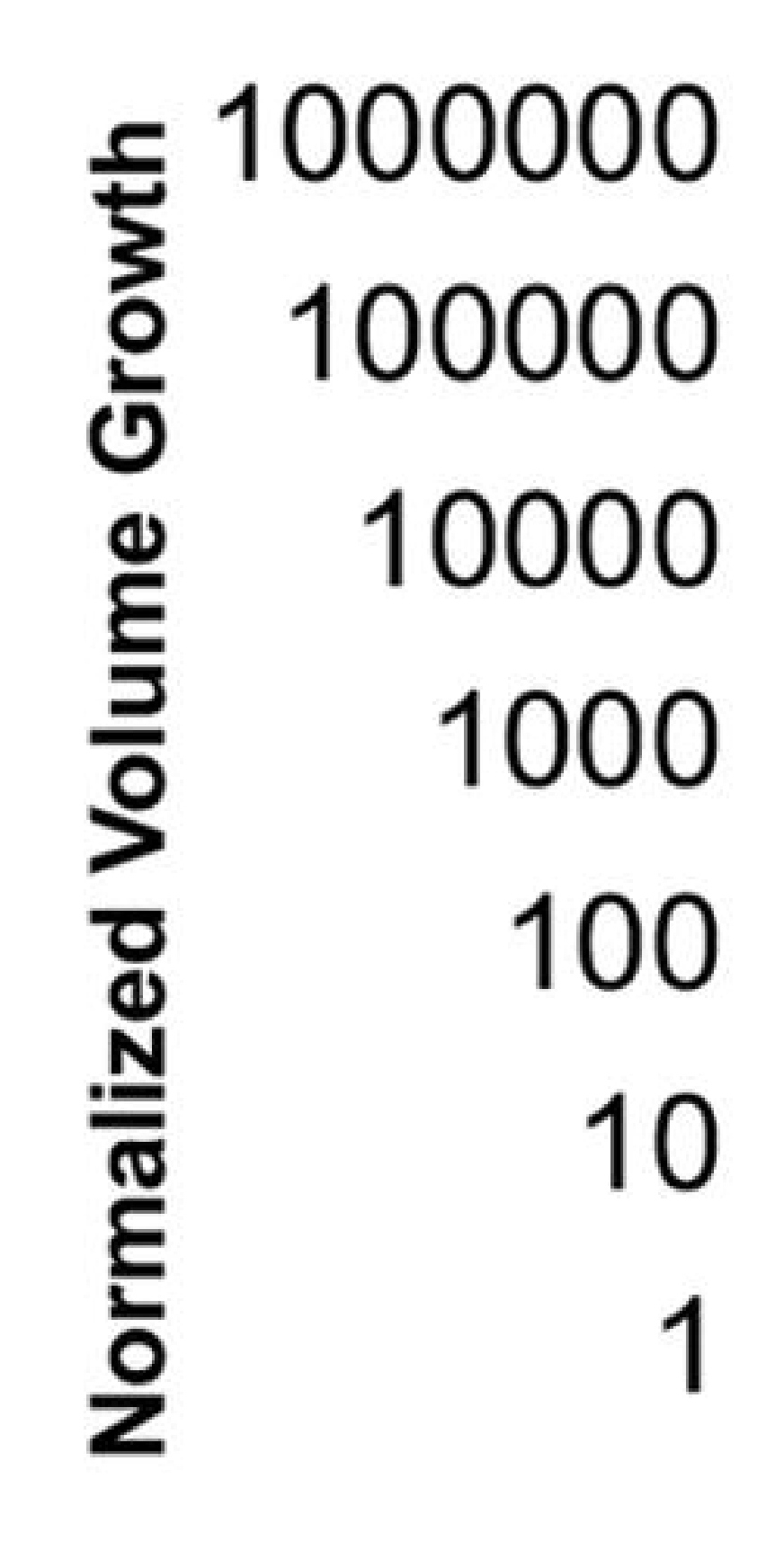

Liver development vs.

Liver regeneration.

- Gompertz Volumetric Model

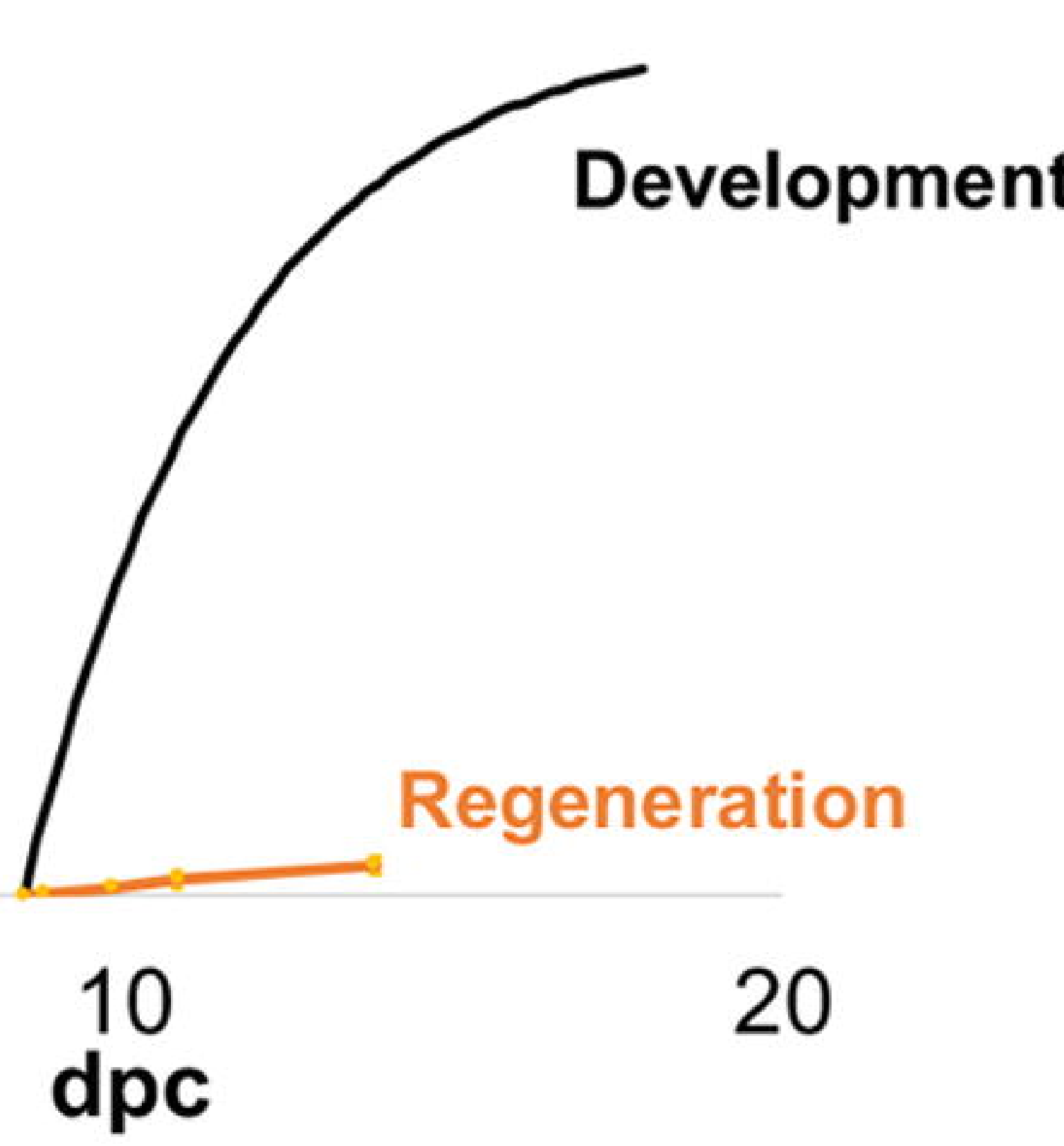

$J$
Posterior
E13

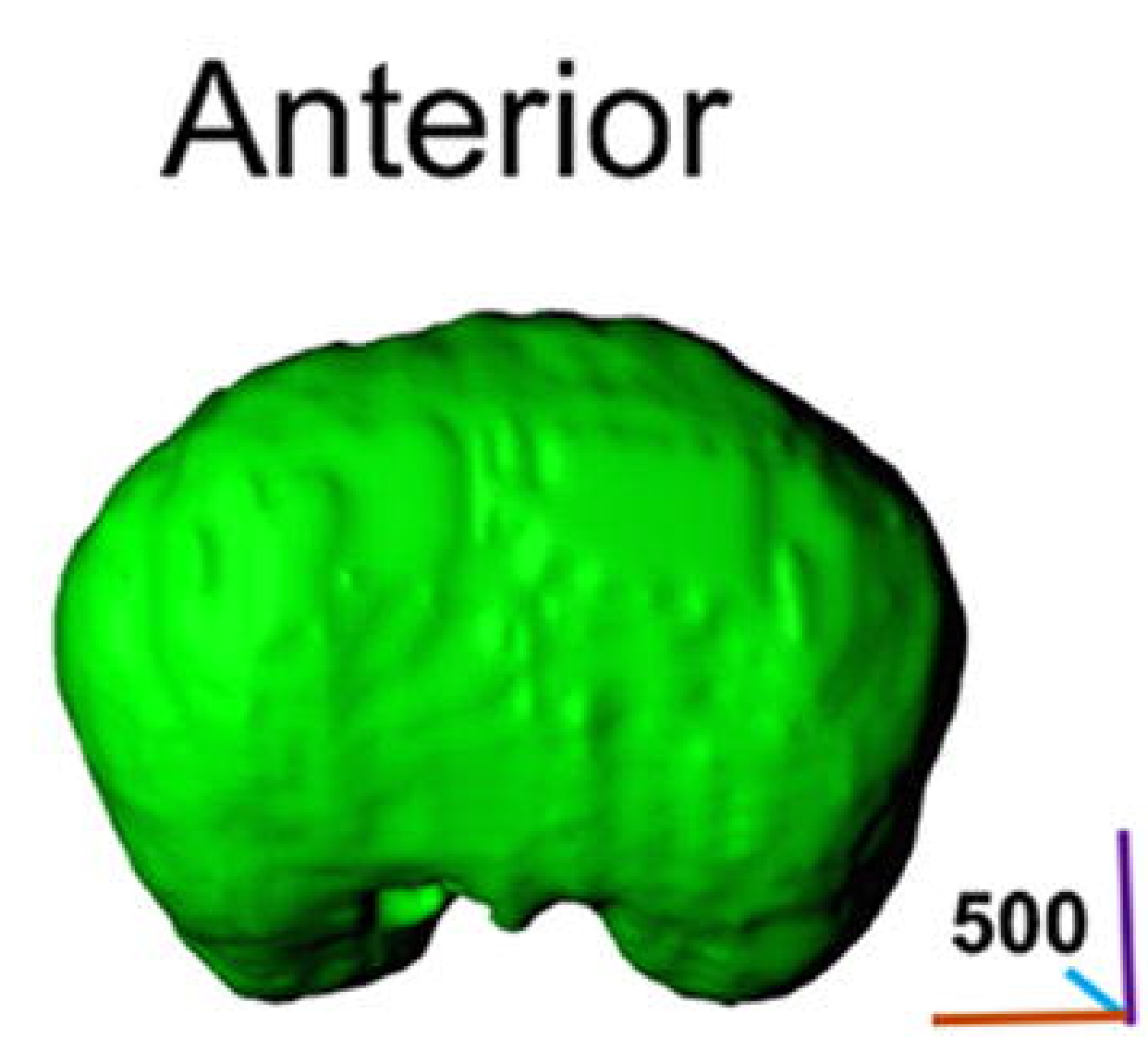

Posterior

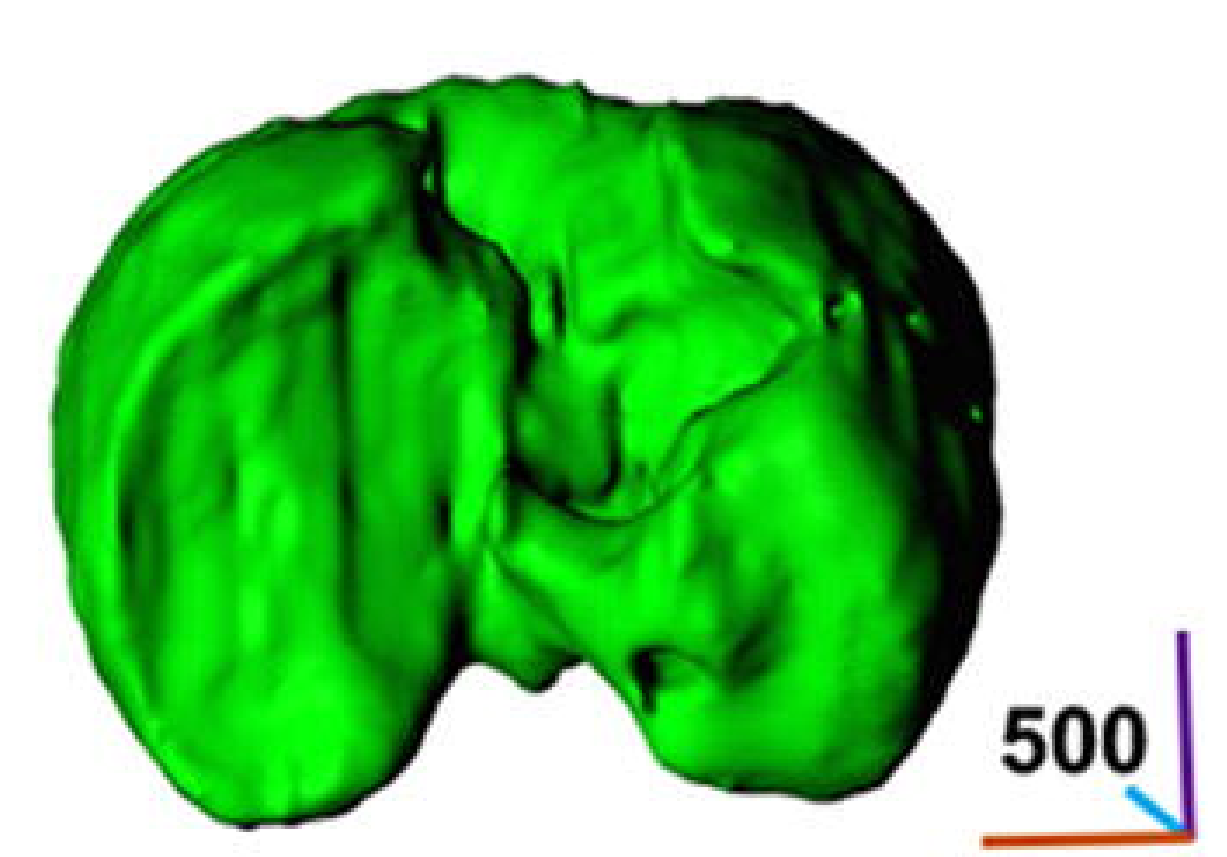

E18
Anterior

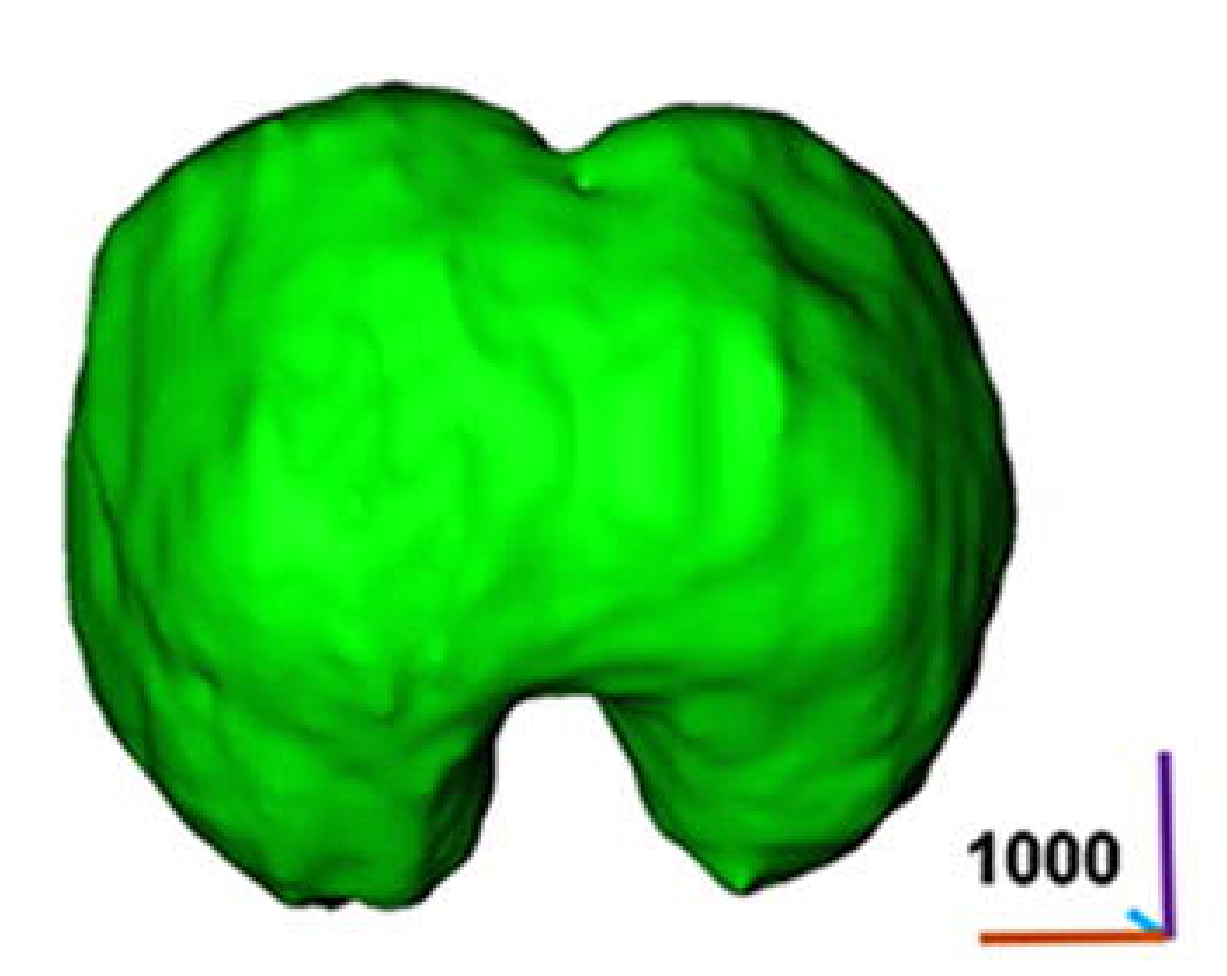




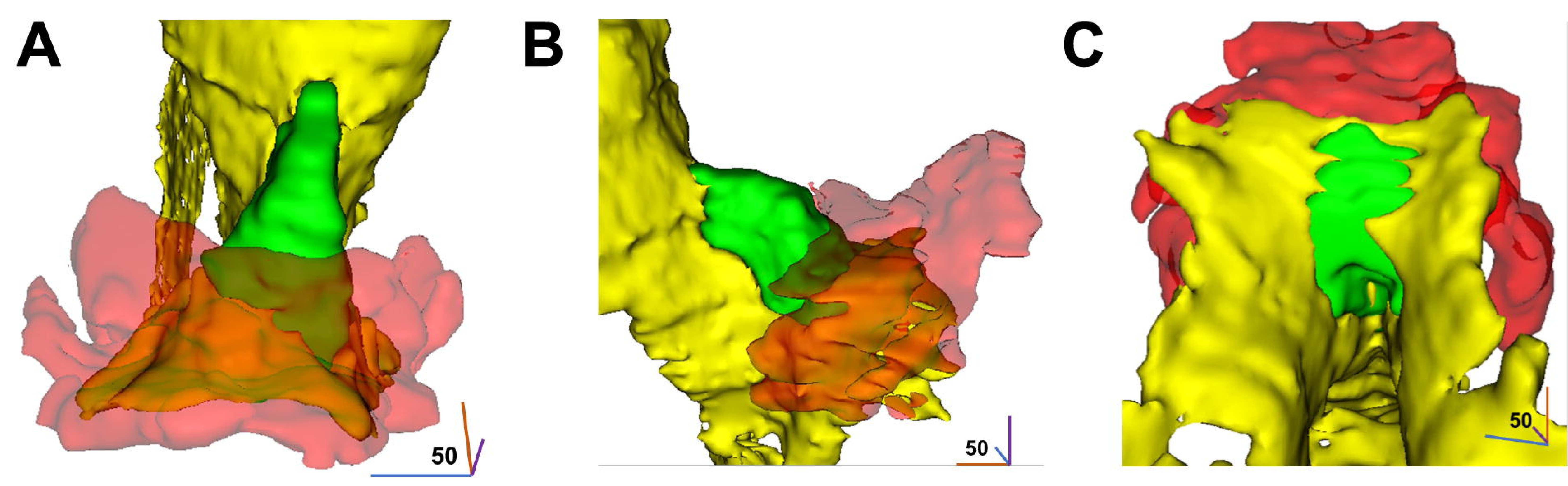

\section{E 8.5}
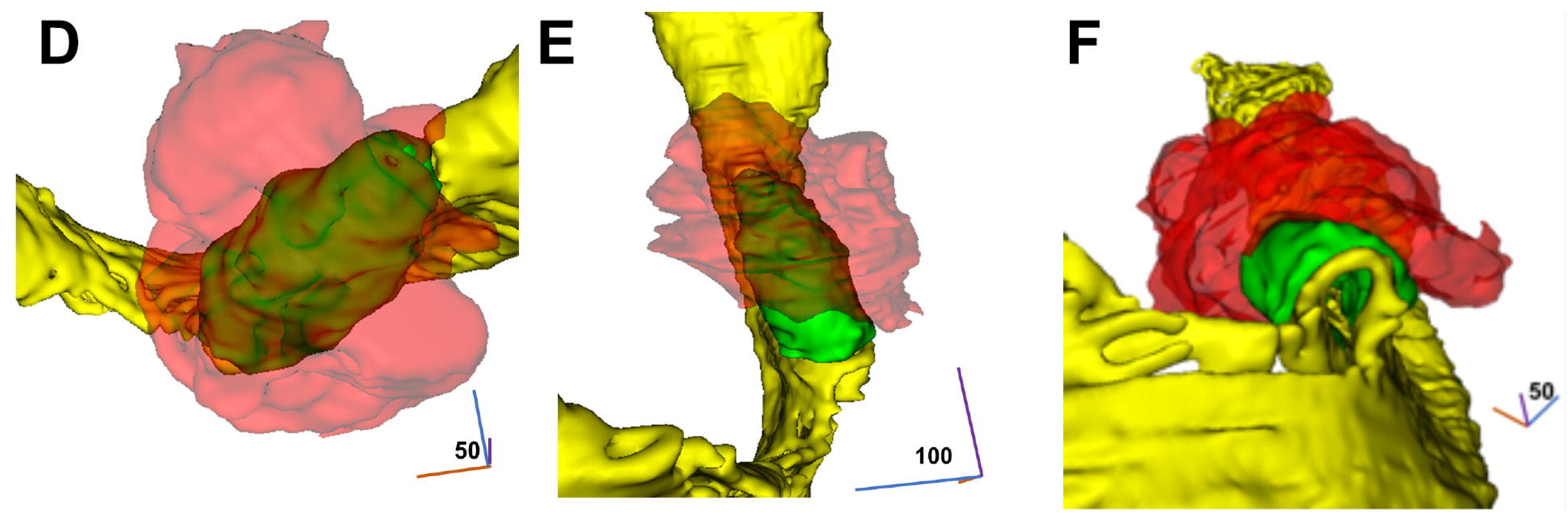

E 9.0
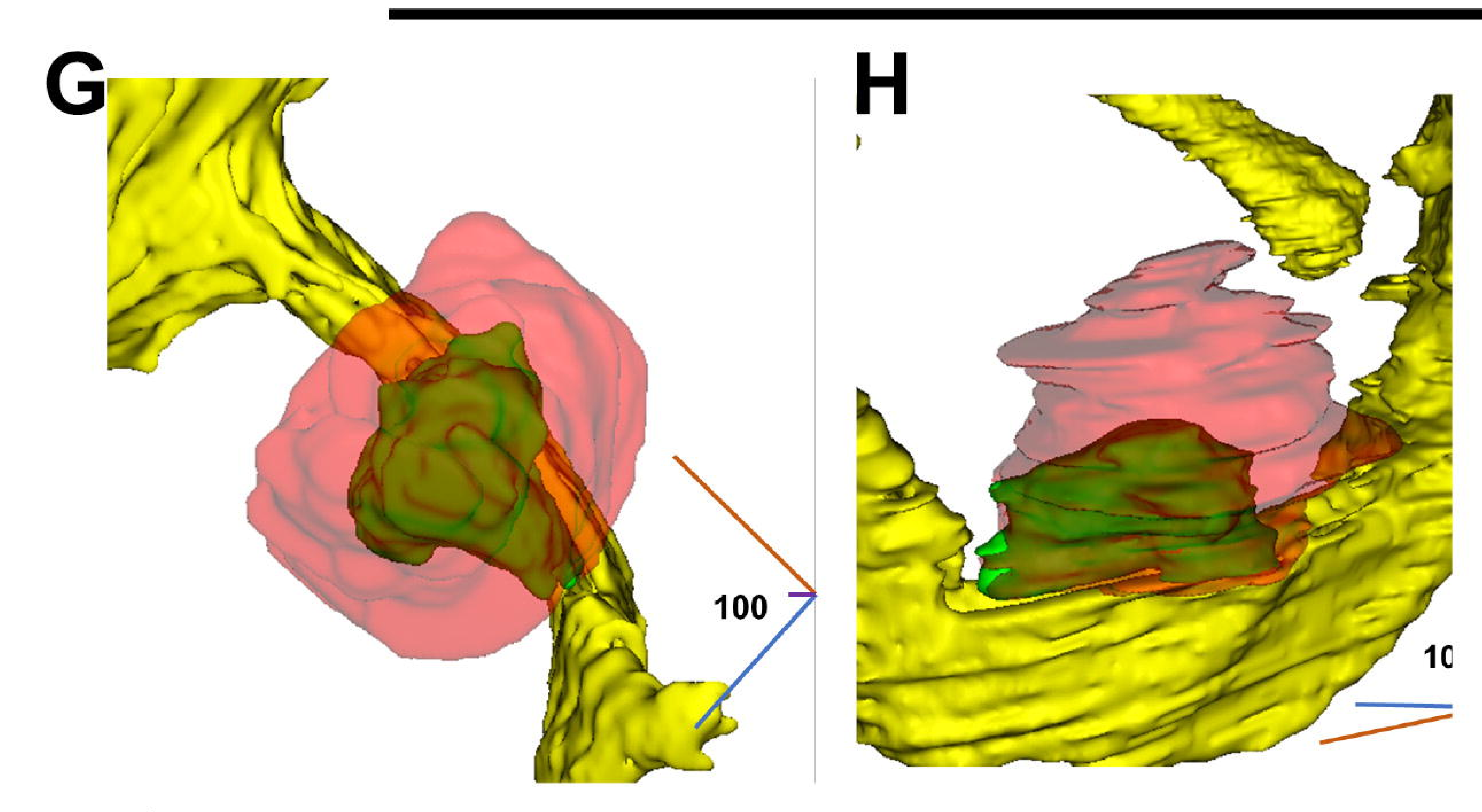

E 9.5

,
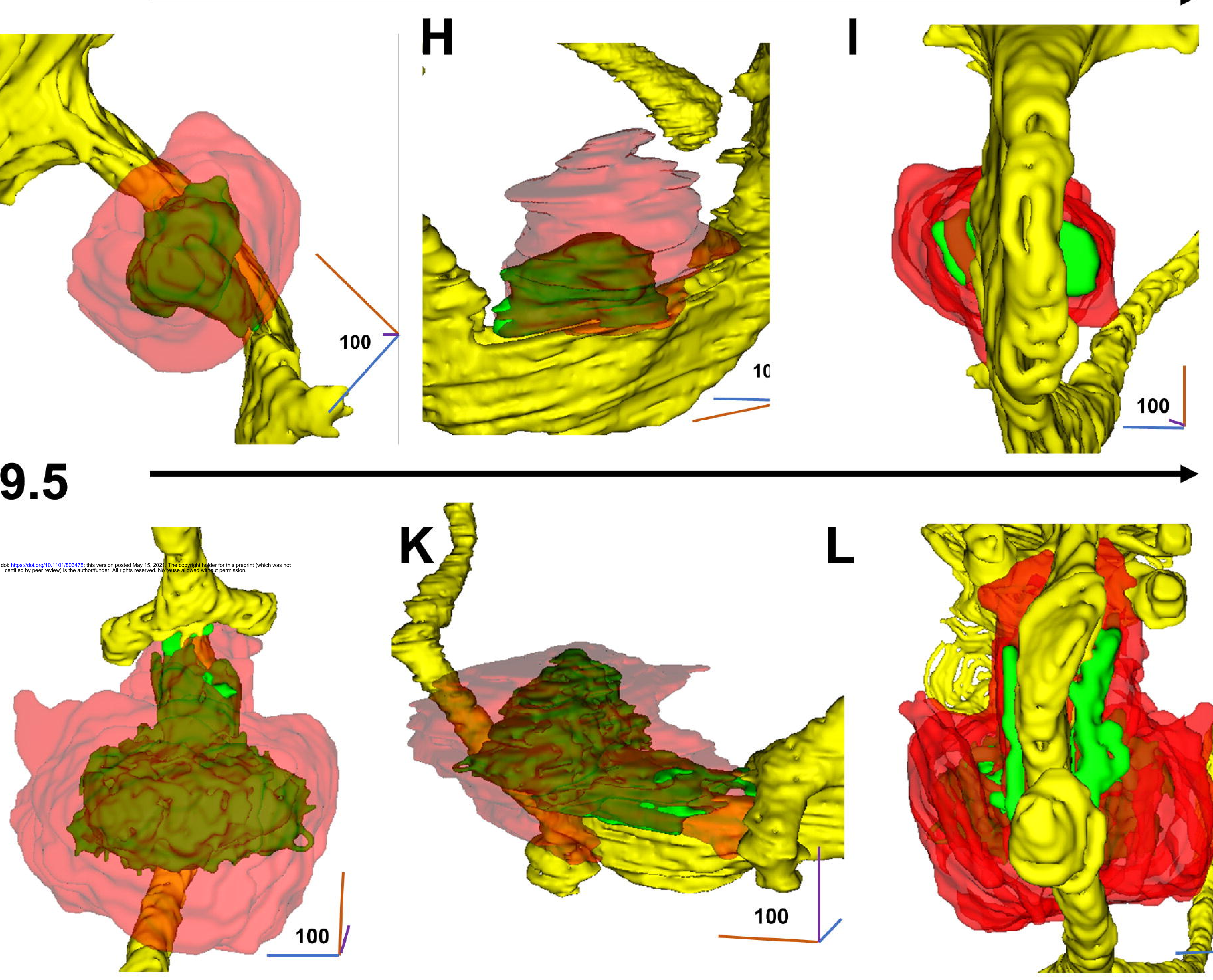


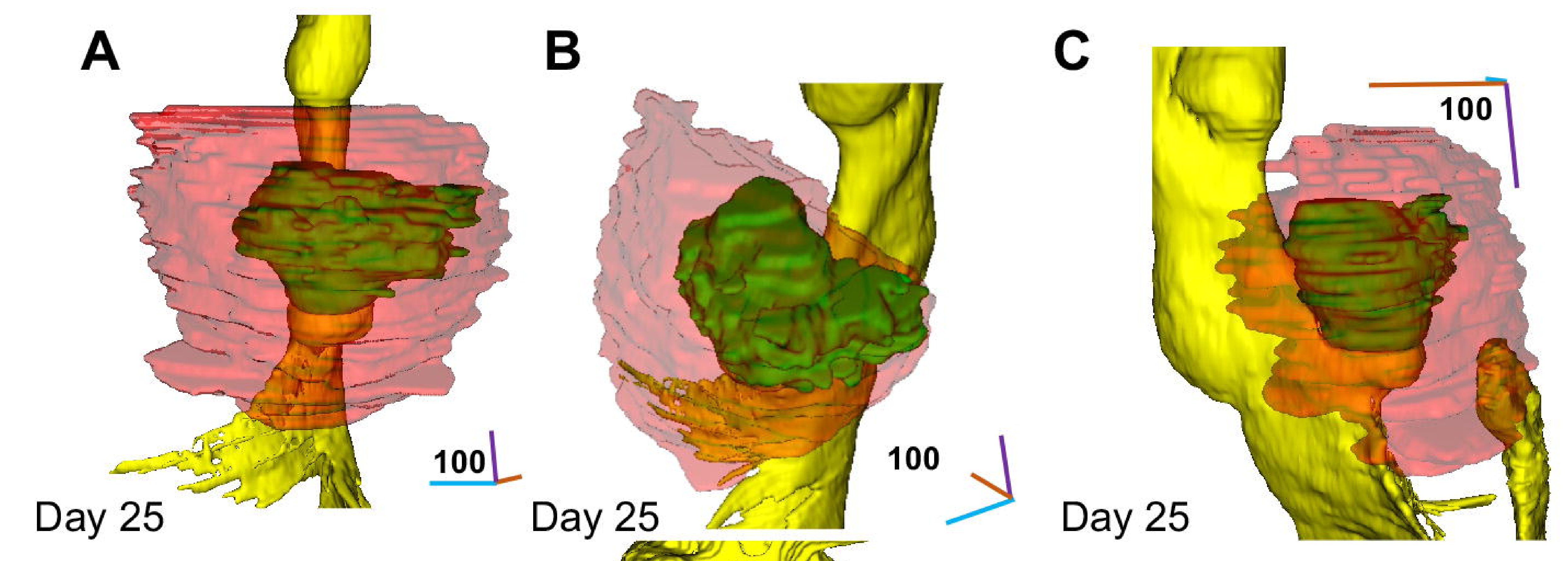

Day 25
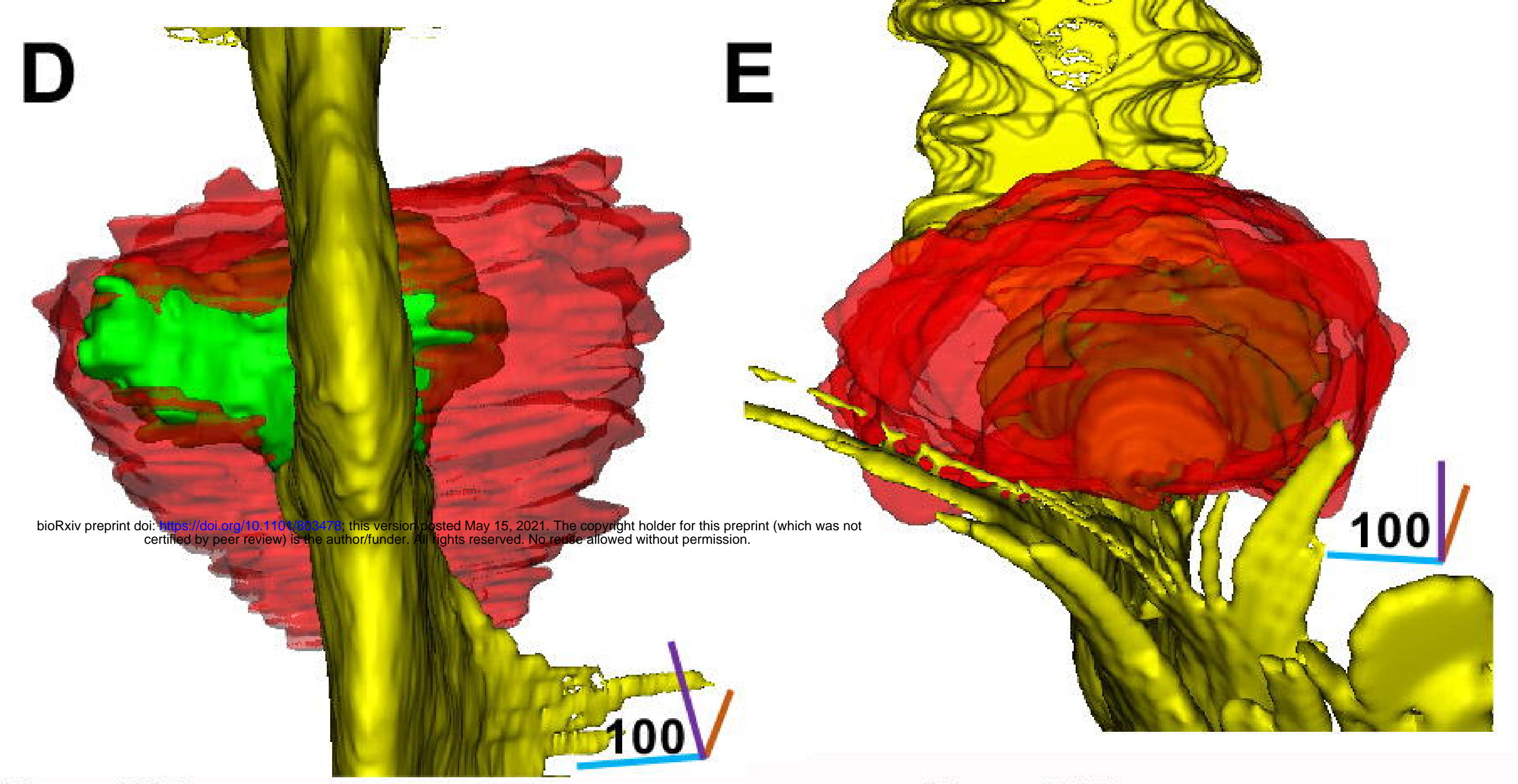

E

Day 25

Day 25

G

Day 28

100

H

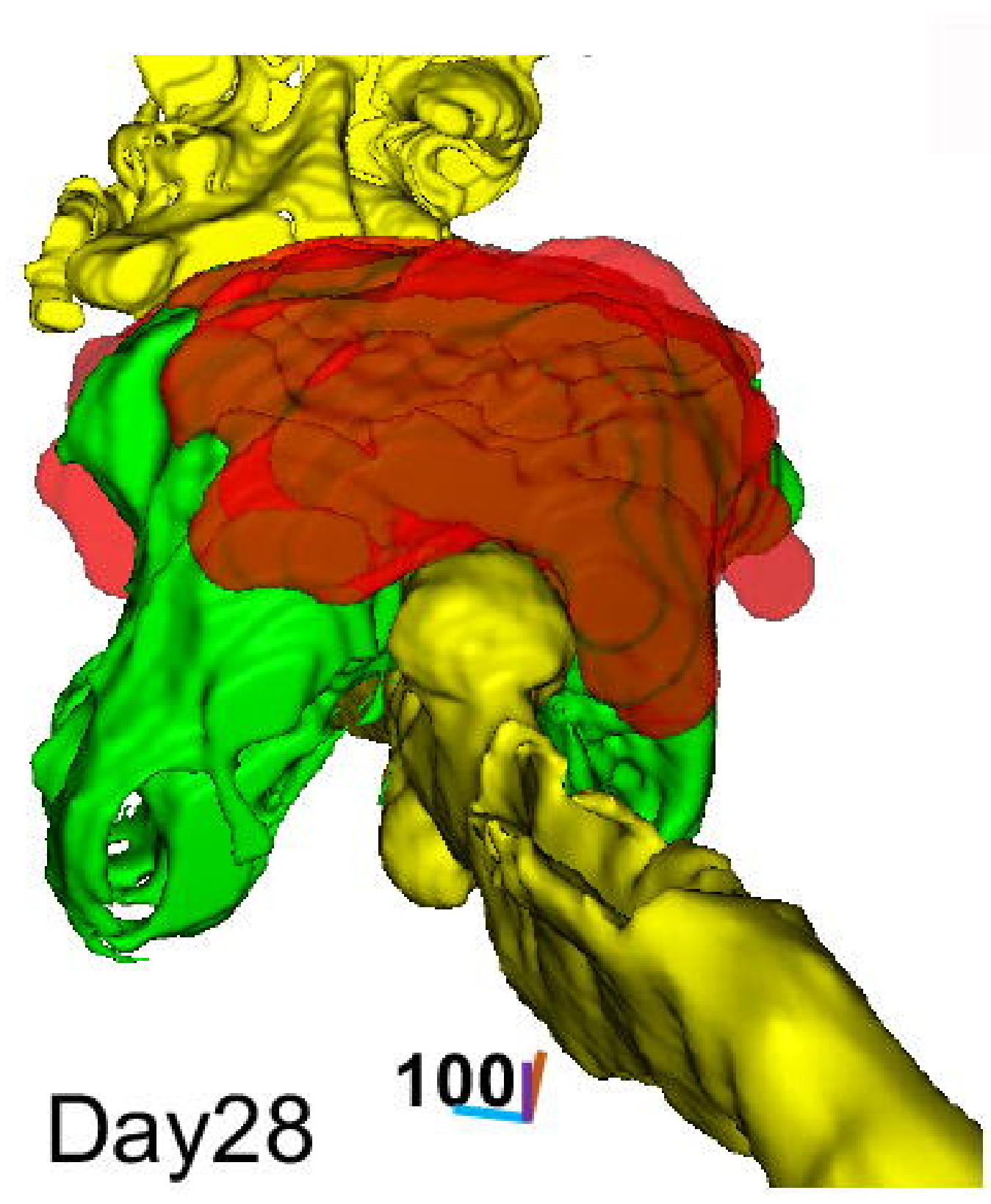

I $\quad 8.00 E+07$

है

$6.00 E+07$

4.00E+07

2.00E+07

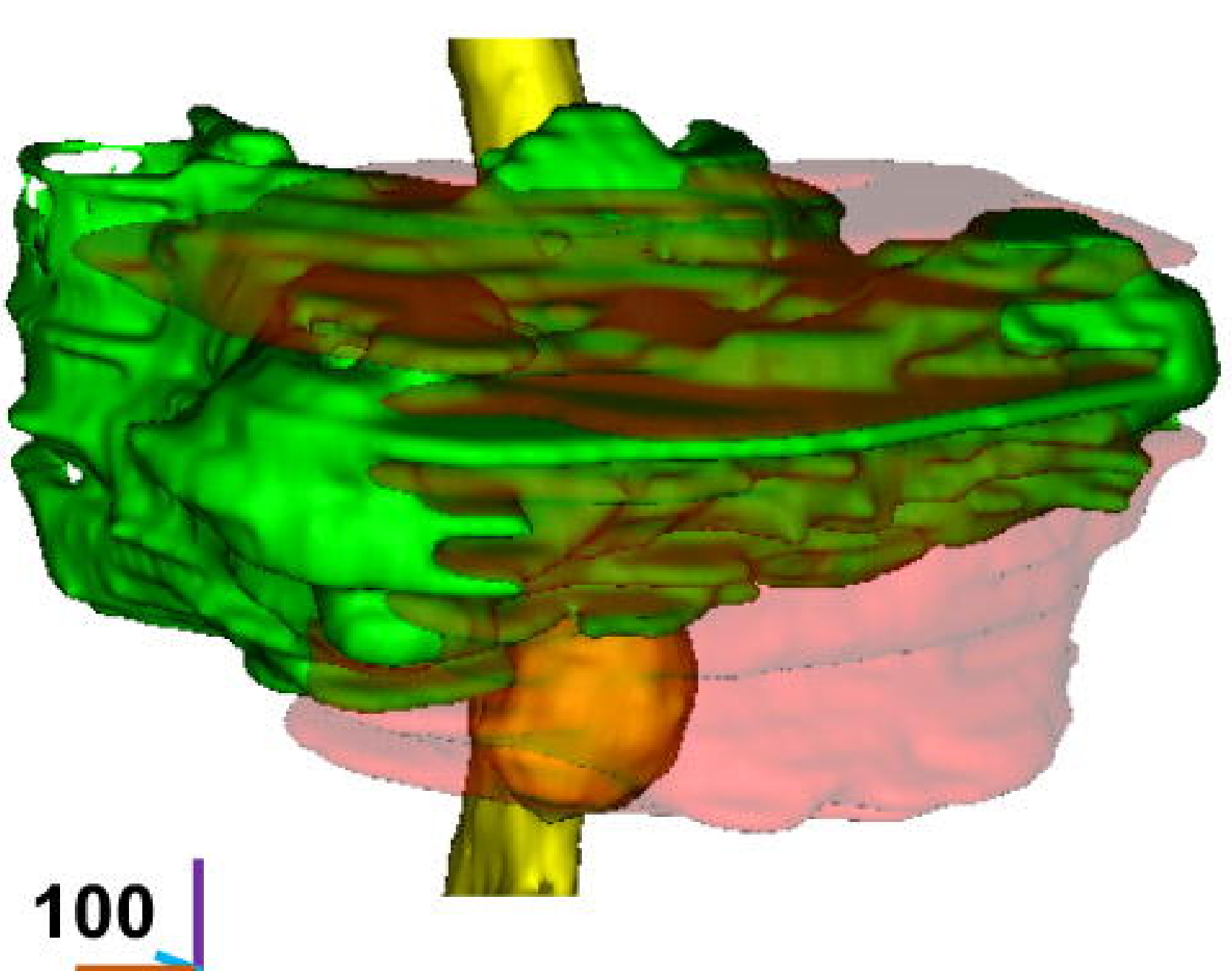

Day 28

$0.00 E+00$

- STM $\square$ Liver

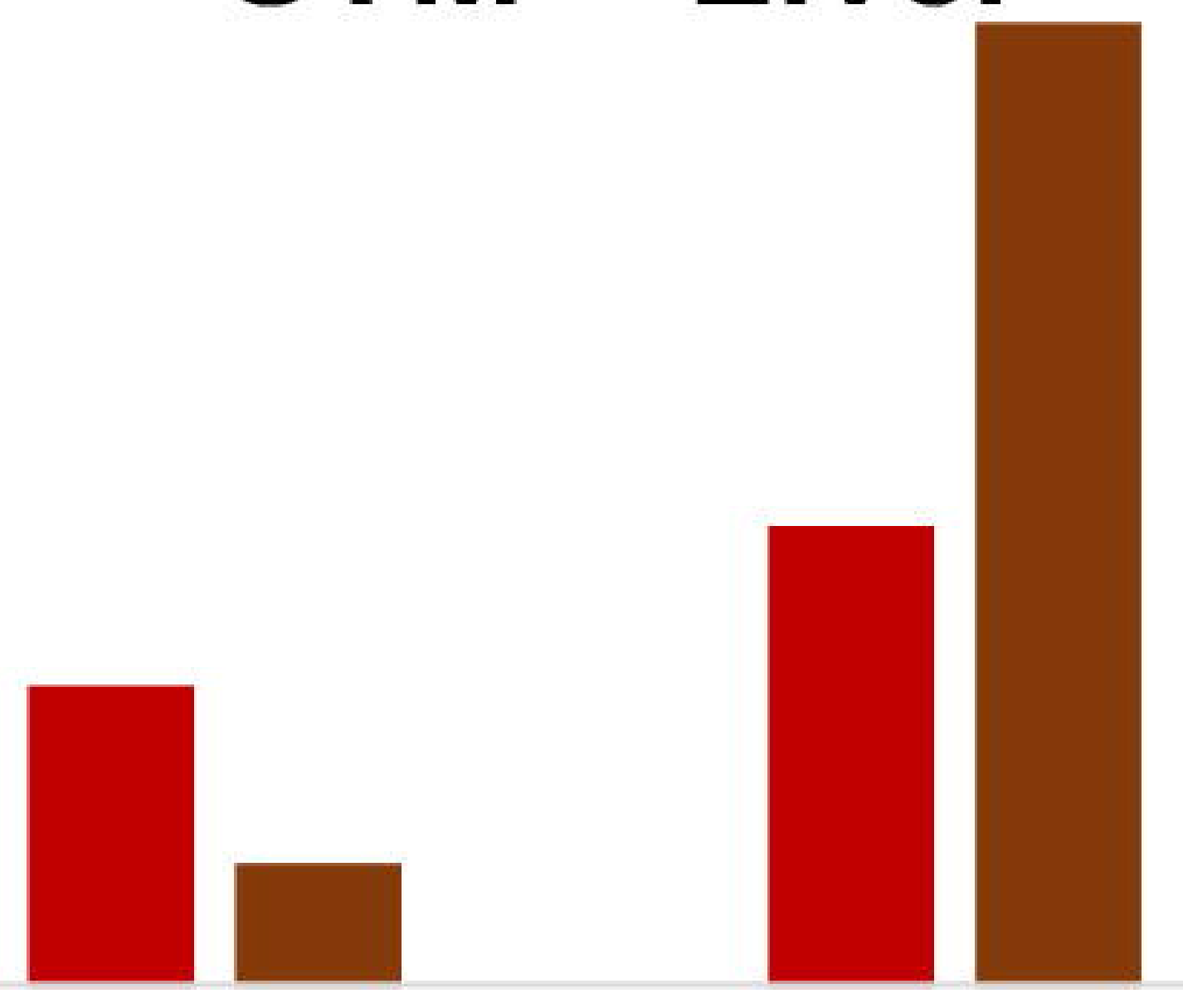

25 dpc 28 
